\title{
British Thoracic Society Guideline for bronchiectasis in adults
}

\author{
Adam T Hill, ${ }^{1}$ Anita L Sullivan, ${ }^{2}$ James D Chalmers, ${ }^{3}$ Anthony De Soyza, ${ }^{4}$ \\ J Stuart Elborn, ${ }^{5}$ R Andres Floto, ${ }^{6,7}$ Lizzie Grillo, ${ }^{8}$ Kevin Gruffydd-Jones, ${ }^{9}$ \\ Alex Harvey, ${ }^{10}$ Charles S Haworth, $^{7}$ Edwin Hiscocks, ${ }^{11}$ John R Hurst, ${ }^{12}$ \\ Christopher Johnson, ${ }^{7}$ W Peter Kelleher, ${ }^{13,14,15}$ Pallavi Bedi, ${ }^{16}$ Karen Payne, ${ }^{17}$ \\ Hashem Saleh, ${ }^{8}$ Nicholas J Screaton, ${ }^{18}$ Maeve Smith, ${ }^{19}$ Michael Tunney, ${ }^{20}$ \\ Deborah Whitters, ${ }^{21}$ Robert Wilson, ${ }^{14}$ Michael R Loebinger ${ }^{14}$
}

For numbered affiliations see end of article.

\section{Correspondence to} Professor Adam T Hill, Respiratory Medicine, Royal Infirmary of Edinburgh, Edinburgh and University of Edinburgh, EH16 4SA, UK; adam.hill318@nhs.net

\section{SUMMARY OF RECOMMENDATIONS AND GOOD PRACTICE POINTS}

How should the diagnosis of bronchiectasis be determined?

Recommendations - Imaging

> Perform baseline chest X-ray in patients with suspected bronchiectasis. (D)

> Perform a thin section computed tomography scan (CT) to confirm a diagnosis of bronchiectasis when clinically suspected. (C)

> Perform baseline imaging during clinically stable disease as this is optimal for diagnostic and serial comparison purposes. (D)

\section{Good practice points}

\section{CT imaging protocol}

$\checkmark$ The most appropriate imaging protocol will vary according to scanner technology and patient factors.

$\checkmark$ When using volumetric CT, dose reduction techniques including adaptive $\mathrm{mA}$ and $\mathrm{kV}$ and iterative reconstruction should be utilised where available.

$\checkmark$ Typical CT imaging parameters for the diagnosis of bronchiectasis are:

- Slice thickness: $\leq 1 \mathrm{~mm}$

o Reconstruction algorithm: - high spatial frequency

- $\mathrm{kVp}: 100-140$

o mAs (or effective mAs): $100-200$

- Gantry rotation time: $<0.5 \mathrm{~s}$

\section{SLinked}

- http://dx.doi.org/thoraxjnl2018-212468

Check for updates

(C) Author(s) (or their employer(s)) 2018. No commercial re-use. See rights and permissions. Published by BMJ.

To cite: Hill AT, Sullivan $A L$ Chalmers JD, et al. Thorax 2019;74 (Suppl 1):1-69.

\section{CT features of bronchiectasis}

$\checkmark$ Bronchiectasis is defined by bronchial dilatation as suggested by one or more of the following:

o Bronchoarterial ratio $>1$ (internal airway lumen vs adjacent pulmonary artery)

o Lack of tapering

o Airway visibility within $1 \mathrm{~cm}$ of costal pleural surface or touching mediastinal pleura.

The following indirect signs are commonly associated with bronchiectasis:

o Bronchial wall thickening

- Mucus impaction

- Mosaic perfusion / air trapping on expiratory CT
General

$\checkmark$ CT scanning can also aid in identifying an aetiology of bronchiectasis eg Allergic bronchopulmonary aspergillosis (ABPA), Non-tuberculous mycobacteria (NTM), primary ciliary dyskinesia, alpha one antitrypsin deficiency, Williams Campbell syndrome and a foreign body.

In whom should the diagnosis of bronchiectasis be suspected?

Recommendations

$>$ Consider investigation for bronchiectasis in patients with persistent production of mucopurulent or purulent sputum particularly with relevant associated risk factors. (D)

$>$ Consider investigation for bronchiectasis in patients with rheumatoid arthritis if they have symptoms of chronic productive cough or recurrent chest infections. (C)

> Consider investigation for bronchiectasis in patients with Chronic Obstructive Pulmonary Disease (COPD) with frequent exacerbations (two or more annually) and a previous positive sputum culture for P. aeruginosa whilst stable. (B)

$>$ Consider investigation for bronchiectasis in patients with inflammatory bowel disease and chronic productive cough. (C)

\section{Good practice points}

$\checkmark$ In at risk groups, if bronchiectasis is suspected, bronchiectasis needs confirmation.

$\checkmark$ In patients with COPD, investigation for bronchiectasis may be appropriate especially in the presence of chronic productive cough with positive sputum cultures for potentially pathogenic microorganisms (PPM) whilst stable or 2 or more exacerbations in the preceding 12 months.

$\checkmark$ In patients with asthma, investigation for bronchiectasis may be appropriate with severe or poorly-controlled disease.

$\checkmark$ In patients with a history of HIV-1 infection, solid organ and bone marrow transplant, and history of immunosuppressive therapy for lymphoma and vasculitis, investigation for bronchiectasis may be appropriate with symptoms of chronic productive cough or recurrent chest infections. 
$\checkmark$ In patients with chronic rhinosinusitis, investigation for bronchiectasis may be appropriate with symptoms of chronic productive cough or recurrent chest infections.

$\checkmark$ In patients with other connective tissue disease or inflammatory bowel disease, investigation for bronchiectasis may be appropriate if they have symptoms such as chronic productive cough or recurrent chest infections.

$\checkmark$ Investigation for bronchiectasis may be appropriate in otherwise healthy individuals with a cough that persists for longer than 8 weeks, especially with sputum production or a history of an appropriate trigger (see BTS Recommendations for the management of cough in adults (61)).

\section{Investigations for causes of bronchiectasis}

Recommendations (see Table 1)

$>$ A panel of investigations should be performed to establish the underlying cause of bronchiectasis. (B)

a. Co-morbidities and past medical history should be recorded in patients diagnosed with bronchiectasis to identify relevant and possibly causative disease such as rheumatoid arthritis, COPD, asthma, gastro-oesophageal reflux disease and inflammatory bowel disease. (C)

b. Measure full blood count, serum total IgE and assessment of sensitisation (specific IgE or skin prick test) to Aspergillus fumigatus in all patients with bronchiectasis. (D)

c. Serum Immunoglobulin G (IgG), Immunoglobulin A (IgA) and Immunoglobulin M (IgM) should be performed in all patients with bronchiectasis. (C)

d. Consider measuring baseline specific antibody levels against capsular polysaccharides of Streptococcus pneumoniae in all patients to investigate for specific antibody deficiency. If pneumococcal antibodies are low, immunise with 23 valent polysaccharide pneumococcal vaccine, followed by measurement of specific antibody levels 4-8 weeks later. (D)

e. Test for cystic fibrosis (according to NICE Guidelines for Cystic Fibrosis (CF)) in patients with supporting clinical features, for example, early onset, male infertility, malabsorption, pancreatitis. (B)

f. Test for Primary Ciliary Dyskinesia (PCD) (according to ERS Guidelines for PCD Diagnosis) in patients with supporting clinical features, including a history of neonatal distress, symptoms from childhood, recurrent otitis media, rhinosinusitis, or infertility. (A)

g. Sputum cultures should be performed in all patients with bronchiectasis for routine and mycobacterial culture. (D)

\section{Good practice points}

$\checkmark$ A previous diagnosis of idiopathic bronchiectasis should prompt careful reinvestigation for a primary cause in the context of a deteriorating clinical course or a young patient (usually considered to be age 50 and under but not limited to this age group).

$\checkmark$ Referral to a specialist centre for investigation should be considered for young patients (usually considered to be age 50 and under but not limited to this age group) and those with apparent idiopathic bronchiectasis especially where there is evidence of progressive disease.

$\checkmark$ Consider testing for rheumatoid factor (RF), anti-cyclic citrullinated peptide (anti CCP), antinuclear antibodies (ANA) and anti-neutrophil cytoplasmic antibodies (ANCA) in patients with coexisting clinical features of arthritis, connective tissue disease and/or systemic vasculitis.

$\checkmark \quad$ Consider testing for alpha 1 antitrypsin (A1AT) deficiency in patients with coexisting basal panacinar emphysema.

$\checkmark$ Investigations for reflux and aspiration should be undertaken only in symptomatic patients, or where there are other suggestive clinical features.

$\checkmark$ Consider bronchoscopy for patients with localised disease to rule out an endobronchial lesion or foreign body as the cause of bronchiectasis.

$\checkmark$ A bronchial aspiration or bronchial wash targeting the areas of bronchiectasis from CT scan of the chest should be considered in patients who do not expectorate and can be particularly helpful in the diagnosis of NTM pulmonary disease.

$\checkmark$ Serum protein electrophoresis should be performed in all patients with bronchiectasis with raised immunoglobulins.

$\checkmark$ Consider HIV-1 serology in patient with bronchiectasis depending on prevalence of HIV-1 and clinical features suggestive of increased risk of retroviral infection.

\section{Research recommendations}

Consensus criteria for diagnosis of ABPA need to be validated in bronchiectasis cohorts.

Consensus criteria for definition of abnormal post pneumococcal test immunisation antibody responses need to be validated in bronchiectasis cohorts.

\section{Severity scoring}

Good practice point

$\checkmark$ Consider using the bronchiectasis severity index which may help guide management.

\section{Stable state treatment}

Which patients should be taught airway clearance techniques?

Recommendation

> Teach individuals with bronchiectasis to perform airway clearance. (D)

\section{Good practice points}

$\checkmark$ Airway clearance techniques should be taught by a respiratory physiotherapist.

$\checkmark$ At initial assessment, a respiratory physiotherapist should educate the patient about their condition and if appropriate give advice on adjuncts (inhaled/oral therapy or exercise) that may enhance effectiveness of their chosen airway clearance technique.

$\checkmark$ Patients admitted with an exacerbation of bronchiectasis should be seen daily by a respiratory physiotherapist until their airway clearance is optimised.

\section{Which airway clearance techniques should be taught? Recommendations}

> Offer active cycle of breathing techniques or oscillating positive expiratory pressure to individuals with bronchiectasis. (D)

> Consider gravity assisted positioning (where not contraindicated) to enhance the effectiveness of an airway clearance technique. (D)

\section{Good practice points}

$\checkmark$ CT imaging should be reviewed to complement the physiotherapy assessment. Where indicated, this information could be used in order to teach the patient the appropriate postural 
drainage position(s) for their affected bronchopulmonary segment(s).

$\checkmark$ Patients should be made aware of the range of available airway clearance techniques.

$\checkmark$ Consider patient preference and adherence when recommending an airway clearance technique.

$\checkmark$ Consider the inclusion of the forced expiration technique (huff) should be considered for all airway clearance techniques.

$\checkmark$ Consider modified postural drainage (no head down tilt) in patients for whom postural drainage is contraindicated or not tolerated.

$\checkmark$ If symptoms of gastroesophageal reflux increase with modified postural drainage (no head down tilt), an airway clearance technique in the sitting position should be taught.

$\checkmark$ Consider autogenic drainage, positive expiratory pressure, high frequency chest wall oscillation and intrapulmonary percussive ventilation as an alternative airway clearance technique if other techniques are not effective or acceptable to the patient.

$\checkmark$ Patients should be encouraged to perform regular physical exercise (plus the forced expiration technique/huff) to promote airway clearance.

$\checkmark \quad$ If there is ongoing haemoptysis, refer back to the respiratory physiotherapist to determine the optimum airways clearance technique.

\section{Airway clearance techniques during an acute exacerbation Good practice points}

$\checkmark$ Manual techniques may be offered to enhance sputum clearance when the patient is fatigued or undergoing an exacerbation.

$\checkmark$ Consider intermittent positive pressure breathing or non-invasive ventilation during an acute exacerbation to offload the work of breathing so fatigued and/or breathless patients can tolerate a longer treatment session and can adopt postural drainage positions.

\section{Research recommendations}

Randomised controlled trials using clinically important outcome measures are required to assess the effectiveness of airway clearance techniques in varying severities of bronchiectasis.

Randomised controlled trials are required to evaluate the effects of airway clearance techniques in patients who are undergoing an exacerbation.

\section{How often should patients carry out airway clearance techniques? \\ Good practice points}

$\checkmark$ The frequency and duration of the airway clearance technique should be tailored to the individual and may alter during periods of exacerbation.

$\checkmark$ Advise individuals to perform their airway clearance technique for a minimum of 10 minutes (up to a maximum of 30 minutes). After this time they should continue until two clear huffs or coughs are completed, or until the patient is starting to become fatigued.

\section{How soon should the patient be reviewed after the initial assessment? \\ Good practice points}

$\checkmark \quad$ Individuals that have been assessed and taught an airway clearance technique should be reviewed by a respiratory physiotherapist within 3 months of their initial assessment.

$\checkmark$ Individuals with bronchiectasis who are followed up in secondary care should be assessed by a respiratory physiotherapist as part of their annual clinical review to ensure their airway clearance regimen is optimised.

$\checkmark$ All individuals with a deterioration in their condition (increased frequency of exacerbations and/or worsening of symptoms) should have their airway clearance technique reviewed by a respiratory physiotherapist (See figure 1 management of the deteriorating patient).

\section{Mucoactives in bronchiectasis}

Recommendations

$>$ Do not routinely use recombinant human DNase in adults with bronchiectasis. (A)

> Consider the use of humidification with sterile water or normal saline to facilitate airway clearance. (D)

\section{Good practice points}

$\checkmark$ Consider a trial of mucoactive treatment in patients with bronchiectasis who have difficulty in sputum expectoration.

$\checkmark$ Perform an airway reactivity challenge test when inhaled mucoactive treatment is first administered.

$\checkmark$ Consider pre-treatment with a bronchodilator prior to inhaled or nebulised mucoactive treatments especially in individuals where bronchoconstriction is likely (patients with asthma or bronchial hyper-reactivity and those with severe airflow obstruction $\mathrm{FEV}_{1}<1$ litre).

$\checkmark$ If carbocysteine is prescribed, a 6 month trial should be given and continued if there is ongoing clinical benefit.

See Figures 3 and 4 and Appendix 2.

\section{Research recommendation}

Randomised controlled trials are needed to assess the long term impact of muco-active therapies.

\section{What is the evidence for long term anti-inflammatory therapies in bronchiectasis?}

Recommendations

> Do not routinely offer inhaled corticosteroids to patients with bronchiectasis without other indications (such as ABPA, chronic asthma, COPD and inflammatory bowel disease). (B)

$>$ Do not offer long-term oral corticosteroids for patients with bronchiectasis without other indications (such as ABPA, chronic asthma, COPD, inflammatory bowel disease). (D)

$>$ Do not routinely offer phosphodiesterase type 4 (PDE4) inhibitors, methylxanthines or leukotriene receptor antagonists for bronchiectasis treatment. (D)

> Do not routinely offer CXCR2 antagonists, neutrophil elastase inhibitors or statins for bronchiectasis treatment. (B)

\section{Good practice point}

$\checkmark$ Inhaled corticosteroids have an established role in the management of asthma and in a proportion of patients with COPD which are common co-morbid conditions in bronchiectasis.

\section{Research recommendation}

Randomised controlled trials are needed to assess the long term impact of anti-inflammatory therapies. 


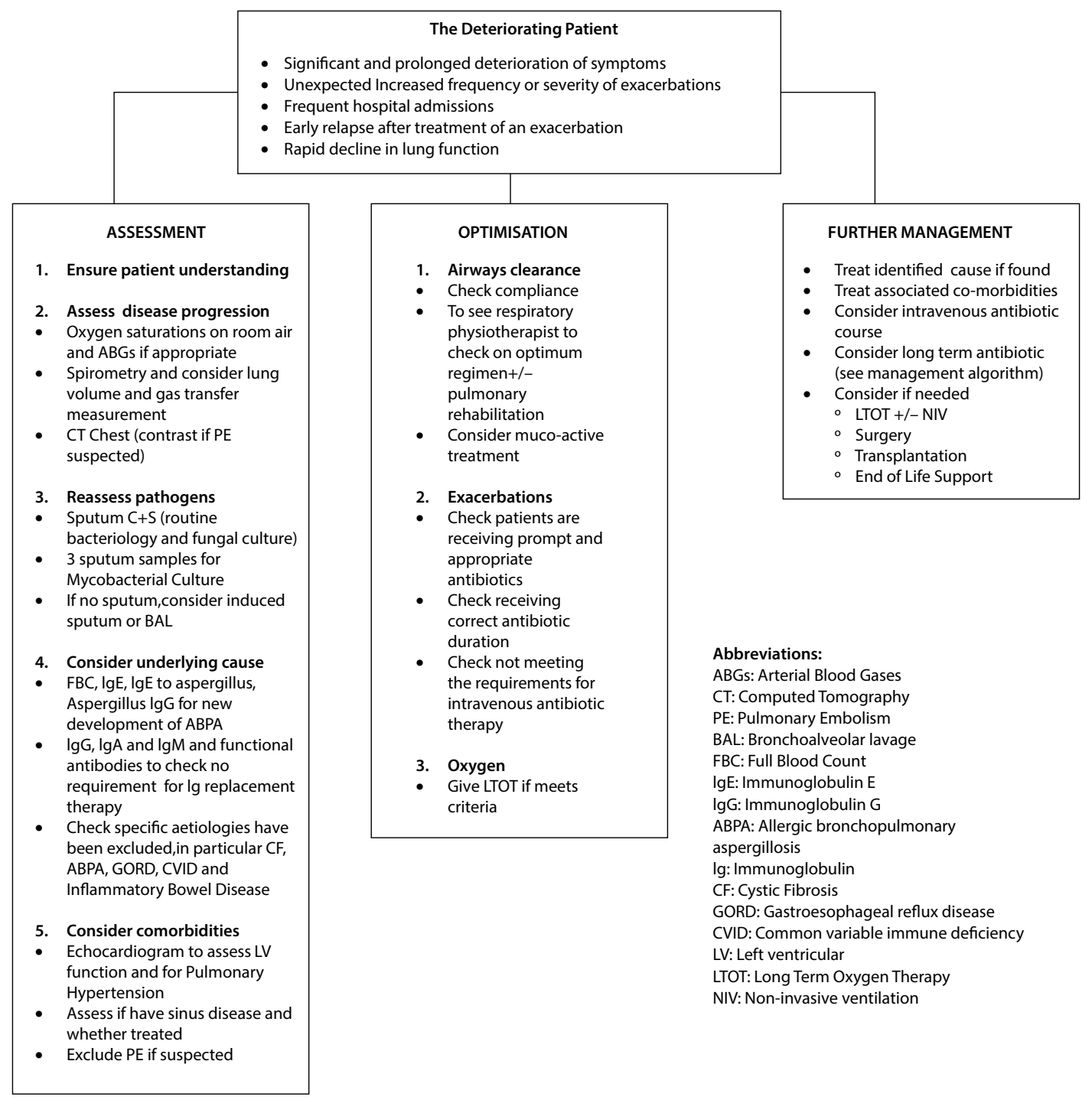

Figure 1 Management of the deteriorating patient.

\section{What treatments improve outcomes for patients with stable bronchiectasis? (see Figure 2 and Appendix 2 and 3 )}

Recommendations

> Consider long term antibiotics in patients with bronchiectasis who experience 3 or more exacerbations per year. (A)

> In these patients, the following are recommended

\section{P. aeruginosa colonised patients}

a. Use inhaled colistin for patients with bronchiectasis and chronic Pseudomonas aeruginosa infection. (B)

b. Consider inhaled gentamicin as a second line alternative to colistin for patients with bronchiectasis and chronic P. aeruginosa infection. (B)

c. Consider azithromycin or erythromycin as an alternative (eg, if a patient does not tolerate inhaled antibiotics) to an inhaled antibiotic for patients with bronchiectasis and chronic P. aeruginosa infection. (B)

d. Consider azithromycin or erythromycin as an additive treatment to an inhaled antibiotic for patients with bronchiectasis and chronic $P$. aeruginosa infection who have a high exacerbation frequency. (D)

\section{Non- $P$. aeruginosa colonised patients}

e. Use azithromycin or erythromycin for patient with bronchiectasis. (A)

f. Consider inhaled gentamicin as a second line alternative to azithromycin or erythromycin. (B)

g. Consider doxycycline as an alternative in patients intolerant of macrolides or in whom they are ineffective. (C)

\section{Good practice points}

$\checkmark$ Antimicrobial stewardship is important

$\checkmark$ Prior to starting long term macrolides, for safety reasons: (1) ensure no active NTM infection with at least one negative respiratory NTM culture; (2) use with caution if the patient has significant hearing loss needing hearing aids or significant balance issues.

$\checkmark$ Prior to starting long term inhaled aminoglycosides, for safety reasons: (1) avoid using if creatinine clearance $<30 \mathrm{ml} / \mathrm{min}$; (2) use with caution if the patient has significant hearing loss needing hearing aids or significant balance issues; (3) avoid concomitant nephrotoxic medications.

$\checkmark$ Counsel patients about potential major side effects with long term antibiotics, and to seek urgent attention if these develop. 


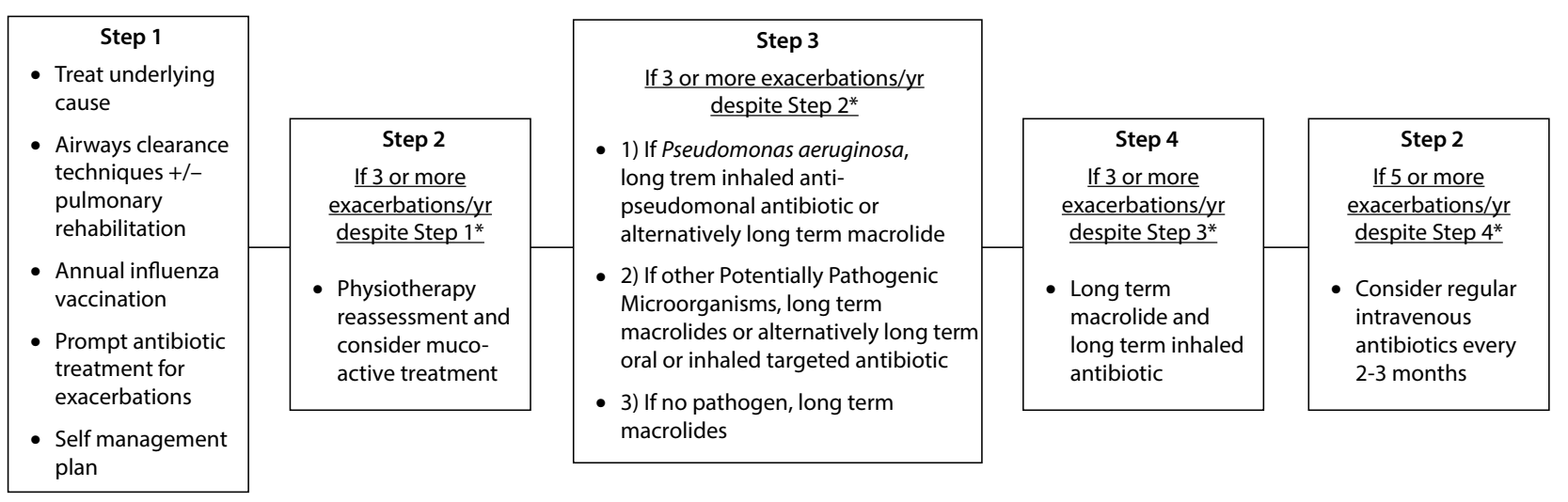

${ }^{*}$ Consider this step if significant symptoms persist despite previous step, even if not meeting exacerbation criteria

Antibiotics are used to treat exacerbations that present with an acute deterioration (usually over several days) with worsening local symtoms ( cough, increased sputum volume or change of viscosity, increased sputum purulence with or without increasing wheeze, breathlessness, haemoptysis) and/or systemic upset. The flow diagram refers to three or more annual exacerbations.

Figure 2 Stepwise management.

a. Review the patient's culture and mycobacterial status, optimise airway clearance and treat other associated conditions before starting long term antibiotics.

b. Prophylactic antibiotics should be only started by respiratory specialists.

c. Review patients on long term antibiotics six monthly with assessment of efficacy, toxicity and continuing need. Monitor sputum culture and sensitivity regularly, although in vitro resistance may not affect clinical efficacy.

$\checkmark$ As adverse event frequency of azithromycin is likely to be dose related, $250 \mathrm{mg} 3 \mathrm{x} /$ week is a pragmatic starting dose which can then be increased according to clinical response and adverse events.

$\checkmark$ Thresholds for long term treatment may reduce if the patient is symptomatic between exacerbations and/or the exacerbations respond poorly to treatment and/or the patient is at high risk of severe exacerbation for example, immunosuppressed.

$\checkmark \quad$ Long term antibiotic choice is complex and has to take into account factors such as tolerance, allergies and sensitivity, therefore in some circumstances, other long term antibiotic regimens may be appropriate (see appendix 3).

$\checkmark$ Perform a suitable challenge test when stable before starting inhaled antibiotics (see appendix 2)

$\checkmark$ Consider cyclical IV antibiotics in patients with repeated infections ( $\geq 5 /$ year) despite other treatments.

$\checkmark$ Alternative inhaled/nebulised agents may become licensed as international studies are completed.

$\checkmark$ For patients receiving long term prophylactic oral antibiotics, the preferred option is to remain on the same antibiotic as opposed to monthly rotation of antibiotics. If there is a subsequent lack of efficacy, the antibiotic can be changed guided by sensitivity results.

\section{Research recommendation}

Long term randomised controlled trials of oral and inhaled antibiotics are needed to assess their efficacy and safety in patients with bronchiectasis who have frequent respiratory tract infections with recurrent $P$. aeruginosa infection or other potential pathogenic micro-organisms.

\section{Does long term bronchodilator treatment improve outcomes for patients with bronchiectasis? Recommendations}

> Use of bronchodilators in patients with bronchiectasis and co-existing COPD or asthma should follow the guideline recommendations for COPD or asthma. (D)

> Offer a trial of long acting bronchodilator therapy in patients with symptoms of significant breathlessness. (D)

> Reversibility testing to beta 2 agonist or anticholinergic bronchodilators may help to identify patients with co-existing asthma but there is no evidence to suggest that a response is required in order to benefit from bronchodilators. (D)

\section{Pulmonary rehabilitation \\ Recommendations}

> Offer pulmonary rehabilitation to individuals who are functionally limited by shortness of breath (Modified Medical Research Council (MMRC) Dyspnoea Scale $\geq 1$ ). (B)

$>$ Consider the use of inspiratory muscle training in conjunction with conventional pulmonary rehabilitation to enhance the maintenance of the training effect. (B)

\section{Good practice points}

$\checkmark$ Educate all individuals with bronchiectasis on the importance of an exercise training programme.

$\checkmark$ Consider the 6 minute walk test (MWT) and/or the incremental shuttle walking test (ISWT) when evaluating exercise capacity pre/post pulmonary rehabilitation in bronchiectasis. Prior to this, practice tests should be carried out to eliminate any learning effect.

$\checkmark$ Pulmonary rehabilitation providers should offer education sessions tailored to the needs of individuals with bronchiectasis (e.g. airway clearance techniques, the pathophysiology of bronchiectasis and relevant inhaled therapy).

$\checkmark$ Pulmonary rehabilitation exercise and education sessions should be provided by appropriately qualified health care practitioners.

Further information on Pulmonary rehabilitation is provided in the BTS Quality Standards for Pulmonary Rehabilitation 
(https://www.brit-thoracic.org.uk/standards-of-care/qualitystandards/bts-pulmonary-rehabilitation-quality-standards/)

\section{Research recommendations}

The role of education, self management plans and who delivers the pulmonary rehabilitation needs to be explored.

The role of pulmonary rehabilitation after exacerbations requiring hospital admission needs to be explored.

The incidence of cross-infection of respiratory pathogens in the group exercise setting should be investigated in the bronchiectasis population.

\section{What is the role of surgery in managing bronchiectasis? Recommendations}

> Consider lung resection in patients with localised disease whose symptoms are not controlled by medical treatment optimised by a bronchiectasis specialist. (D)

> Offer multidisciplinary assessment, including a bronchiectasis physician, a thoracic surgeon and an experienced anaesthetist, of suitability for surgery and pre-operative assessment of cardiopulmonary reserve post resection. (D)

Good practice point

$\checkmark$ Consider nutritional support and pre-operative pulmonary rehabilitation before surgical referral.

\section{Lung transplantation for bronchiectasis}

\section{Recommendations}

$>$ Consider transplant referral in bronchiectasis patients aged 65 years or less if the $\mathrm{FEV}_{1}$ is $<30 \%$ with significant clinical instability or if there is a rapid progressive respiratory deterioration despite optimal medical management. (D)

> Consider earlier transplant referral in bronchiectasis patients with poor lung function and the following additional factors: massive haemoptysis, severe secondary pulmonary hypertension, ICU admissions or respiratory failure (particularly if requiring NIV).(D)

\section{Good practice points}

$\checkmark$ Discuss appropriate patients with a transplant centre prior to formal referral.

$\checkmark$ Optimise management of comorbidities such as osteoporosis and maintain physical condition through pulmonary rehabilitation prior to transplant.

\section{What is the role of influenza and pneumococcal vaccination in management of bronchiectasis \\ Recommendations}

> Offer annual influenza immunisation to all patients with bronchiectasis. (D)

> Offer polysaccharide pneumococcal vaccination to all patients with bronchiectasis. (D)

\section{Good practice point}

$\checkmark$ Consider influenza vaccination in household contacts of patients with immune deficiency and bronchiectasis to reduce the risks of secondary transmission.

$\checkmark$ Consider use of 13 valent protein conjugate pneumococcal vaccine in patients with bronchiectasis who do not have an appropriate serological response to standard polysaccharide vaccine (23 valent carbohydrate Pneumococcal vaccine).

\section{Treatment of respiratory failure}

Recommendations

> Consider long term oxygen therapy for patients with bronchiectasis and respiratory failure, using the same eligibility criteria as for COPD. (D)

> Consider domiciliary non-invasive ventilation with humidification for patients with bronchiectasis and respiratory failure associated with hypercapnia, especially where this is associated with symptoms or recurrent hospitalisation. (D)

\section{Bronchiectasis and other treatments Recommendation}

> Do not routinely recommend alternative treatments (for example cough suppression, nutritional supplementation, complementary therapy/homeopathy, supplemental treatments) as part of the management of patients with bronchiectasis. (D)

Good practice point

$\checkmark$ Record patient's weight and BMI at each clinic appointment.

\section{Research recommendations}

Further interventional/randomised controlled trials needed to establish the role of any alternative therapies in the management of bronchiectasis.

Studies assessing the benefits of nutritional supplementation in patients with bronchiectasis should be undertaken.

\section{Do pathogens have an impact on prognosis in bronchiectasis? \\ Recommendations}

> Consider patients with chronic $P$. aeruginosa colonisation at higher risk of bronchiectasis-related complications. (B)

> Perform regular sputum microbiology screening for patients with clinically significant bronchiectasis to monitor for pathogens and detect new isolation of $P$. aeruginosa. (C)

\section{What is the evidence for the role of viruses/fungal disease in patients with bronchiectasis? \\ Good practice points \\ $\checkmark$ Testing to detect viral infection should be considered in patients with an exacerbation of bronchiectasis. \\ $\checkmark$ Do not routinely use anti-fungal therapy without evidence of fungal disease. Fungal cultures can be positive on those receiving long-term antibiotic therapy.}

\section{Does eradication of potentially pathogenic microorganisms improve outcomes in patients with stable bronchiectasis? Recommendations}

> Offer patients with bronchiectasis associated with clinical deterioration and a new growth of $P$. aeruginosa (1st isolation or regrowth in the context of intermittently positive cultures) eradication antibiotic treatment. (first line treatment: ciprofloxacin 500-750 mg bd for 2 weeks; second line treatment: iv antipseudomonal beta-lactam \pm aniv aminoglycoside for 2 weeks, followed by a 3 month course of nebulised colistin, gentamicin or tobramycin). (D)

$>$ Discuss with patients the potential risks and benefits of starting eradication antibiotic treatment versus clinical 
observation following a new growth of $P$. aeruginosa in the context of stable bronchiectasis. This will include consideration of the likelihood of achieving sustained eradication, the risk of developing chronic infection, and the risk of adverse events with each management approach. (D)

> Offer patients with bronchiectasis associated with clinical deterioration and a new growth of methicillin-resistant $S$. aureus (MRSA) (1st isolation or regrowth in the context of intermittently positive cultures) eradication. This should be attempted especially in view of infection control issues. (D)

Good practice point

$\checkmark$ Send sputum for culture and sensitivity immediately before and at each clinical attendance following antibiotics prescribed for bacterial eradication to determine the outcome of treatment.

\section{Research recommendation}

A randomised control trial of $P$. aeruginosa eradication therapy is needed to determine the microbiological and clinical outcomes of eradication therapy.

\section{Does antibiotic therapy improve outcomes in patients with an exacerbation of bronchiectasis? \\ Good practice points}

$\checkmark$ A patient self management plan should be considered, an example is provided here https:/www.brit-thoracic. org.uk/standards-of-care/quality-standards/bts-bronchiectasisquality-standards/)

$\checkmark$ There should be prompt treatment of exacerbations and suitable patients should have antibiotics to keep at home.

$\checkmark$ Previous sputum bacteriology results can be useful in deciding which antibiotic to use. Table 6 highlights the first-line and alternative treatments for the common bacterial pathogens implicated in exacerbations of bronchiectasis.

$\checkmark$ Where possible, sputum (spontaneous or induced) should be obtained for culture and sensitivity testing prior to commencing antibiotics.

$\checkmark$ Empirical antibiotics can then be started while awaiting sputum microbiology.

$\checkmark$ Once a pathogen is isolated, antibiotics can be modified if there is no clinical improvement, with treatment guided by antibiotic sensitivity results.

$\checkmark \quad$ In general, antibiotic courses for 14 days are standard and should always be used in patients infected with $P$. aeruginosa. Shorter courses may suffice in patients with mild bronchiectasis.

$\checkmark \quad$ Intravenous antibiotics should be considered when patients are particularly unwell, have resistant organisms or have failed to respond to oral therapy (this is most likely to apply to patients with $P$. aeruginosa).

\section{What treatments improve outcomes in patients with bronchiectasis and allergic broncho-pulmonary aspergillosis? \\ Recommendations}

$\checkmark$ Offer oral corticosteroid to patients with active ABPA. An initial dose of $0.5 \mathrm{mg} / \mathrm{kg} / \mathrm{d}$, for 2 weeks is recommended. Wean steroids according to clinical response and serum IgE levels. (D) $\checkmark$ Consider itraconazole as a steroid sparing agent for patients dependent on oral corticosteroids where difficulty in weaning is experienced. (B)

$\checkmark$ Monitor patients with active ABPA with total IgE level to assess treatment response. (C)

\section{Does immunoglobulin replacement treatment therapy} improve outcomes in patients with bronchiectasis due to antibody deficiency?

Recommendations

> Offer IgG therapy to all patients with common variable immune deficiency (CVID) and $\mathrm{x}$ linked agammaglobulnemia (XLA). (B)

> Consider IgG therapy for patients with specific polysaccharide antibody deficiency and/or IgA deficiency or IgG subclass deficiencies with absent/impaired pneumococcal vaccine antibody responses who continue to have objective evidence of bacterial sino-pulmonary infection and progressive disease despite appropriate management of bronchiectasis. (C)

\section{Good practice points}

$\checkmark$ All patients receiving IgG replacement therapy should be under the joint care of a clinical immunologist and respiratory specialist in bronchiectasis

$\checkmark$ Offer patients receiving replacement IgG the choice of hospital or home-based therapy.

\section{Research recommendation}

Randomised controlled trials are needed to assess which patients with bronchiectasis would benefit from long term Immunoglobulin $G$ replacement therapy alone or as an adjunct to long term antibiotic therapy- assessing the optimal dose of IgG replacement and identification of ideal trough IgG level to prevent recurrent infections.

\section{Gastro-oesophageal reflux disease (GORD) and bronchiectasis} Recommendation

$>$ Treat GORD symptoms in patients with bronchiectasis according to existing NICE guidance ${ }^{319}$. (D)

\section{Good practice points}

$\checkmark$ GORD should be considered in patients with hiatus hernia, persistent coliforms in sputum samples or recurrent exacerbations with no other cause identified.

$\checkmark \quad$ Investigate patients who may have gastro-oesophageal reflux according to local policies.

$\checkmark$ Consider the addition of prokinetic agents if symptoms of GORD increase with an airway clearance technique in sitting position. Avoid eating in the hour immediately prior to physiotherapy.

\section{What is the prevalence of rhinosinusitis in patients with} stable bronchiectasis and what are the outcomes of treatment?

\section{Recommendations}

> The evaluation of patients with bronchiectasis should include assessment of symptoms of chronic rhinosinusitis. (D)

$>$ Patients with bronchiectasis and symptoms of rhinosinusitis should be evaluated and treated according to existing evidence-based treatment pathways. (D) 


\section{Should treatment of bronchiectasis be altered in the presence of co-morbidities? \\ Recommendations}

> Consider a trial of inhaled and/or oral corticosteroids in patients with bronchiectasis and inflammatory bowel disease (IBD). (D)

$>$ Ensure optimal control of asthma and allergies in patients with both bronchiectasis and asthma (D).

$>$ Monitor patients with co-morbid COPD and bronchiectasis as they are at higher risk of death. (D)

> Patients with bronchiectasis and autoimmune conditions should be carefully assessed for autoimmune related lung disease and often require long term follow up in a secondary care setting. (D)

> Patients with bronchiectasis who require disease modifying antirheumatic drugs (DMARDs) or biologics for rheumatoid arthritis should be referred to a chest physician for further assessment before treatment is started. (D)

\section{How should we monitor bronchiectasis?}

\section{Recommendation}

> All patients with bronchiectasis should undergo routine monitoring in order to identify disease progression, pathogen emergence and modify treatment where necessary. (D)

\section{Good practice points}

$\checkmark$ Tailor the frequency of routine monitoring to the patient's disease severity (see table 7).

$\checkmark$ Assess patients annually, and more frequently in more severe disease.

$\checkmark \quad$ Perform pulse oximetry to screen for patients who may need blood gas analysis to detect respiratory failure.

$\checkmark$ A baseline CXR may provide a useful comparator in the event of clinical deterioration.

\section{Is there a role for microbiological sensitivity testing?}

\section{Good practice points}

$\checkmark$ Antibiotic sensitivity testing can be used to determine if resistance develops to either acute or long-term antibiotic treatment.

$\checkmark$ Where possible, treatment should be guided by antibiotic sensitivity results but is often empirical based on previous sputum bacteriology.

$\checkmark$ Some patients with an infective exacerbation may respond to antibiotic treatment despite resistance to that drug in vitro.
Antibiotics should only be changed if there is no clinical response.

$\checkmark$ For those on long term antibiotic treatment, there should be repeat sensitivity testing where there is a clinical concern regarding loss of efficacy with therapy.

\section{Is there any evidence of cross-infection with pathogenic organisms (conventional bacteria and environmental mycobacteria)?}

Recommendations

> Individual or cohort segregation based on respiratory tract microbiology results is not routinely required for patients with bronchiectasis. (D)

\section{Good practice points}

$\checkmark$ Good cross infection prevention principles should be applied: seek advice on local policies.

$\checkmark \quad$ The transmissibility of P. aeruginosa in cystic fibrosis appears more common. In the case of shared facilities with cystic fibrosis patients the cross infection guidelines for cystic fibrosis should prevail.

\section{Research recommendation}

Large scale robust data that confirm or refute the transmissibility of key pathogens such as $P$. aeruginosa and non-tuberculous mycobacteria are needed.

\section{Specialist vs non-specialist setting}

Good practice point

$\checkmark$ Specialist clinics should be considered in patients requiring hospital follow up.

\section{What are the complications of bronchiectasis?}

Good practice point

$\checkmark$ If haemoptysis $10 \mathrm{mls}$ or less over a 24 hour period, treat with an appropriate oral antibiotic. If clinical deterioration, arrange emergency admission to hospital.

$\checkmark$ Management of major haemoptysis should be multidisciplinary with involvement of respiratory physicians, interventional radiology and thoracic surgeons. Empirically treat patients with intravenous antibiotic therapy, based on their known microbiology, and consider adjunct treatment with tranexamic acid. Bronchial artery embolisation is the recommended first line treatment if significant haemoptysis persists. 
Table 1 Quick summary guide

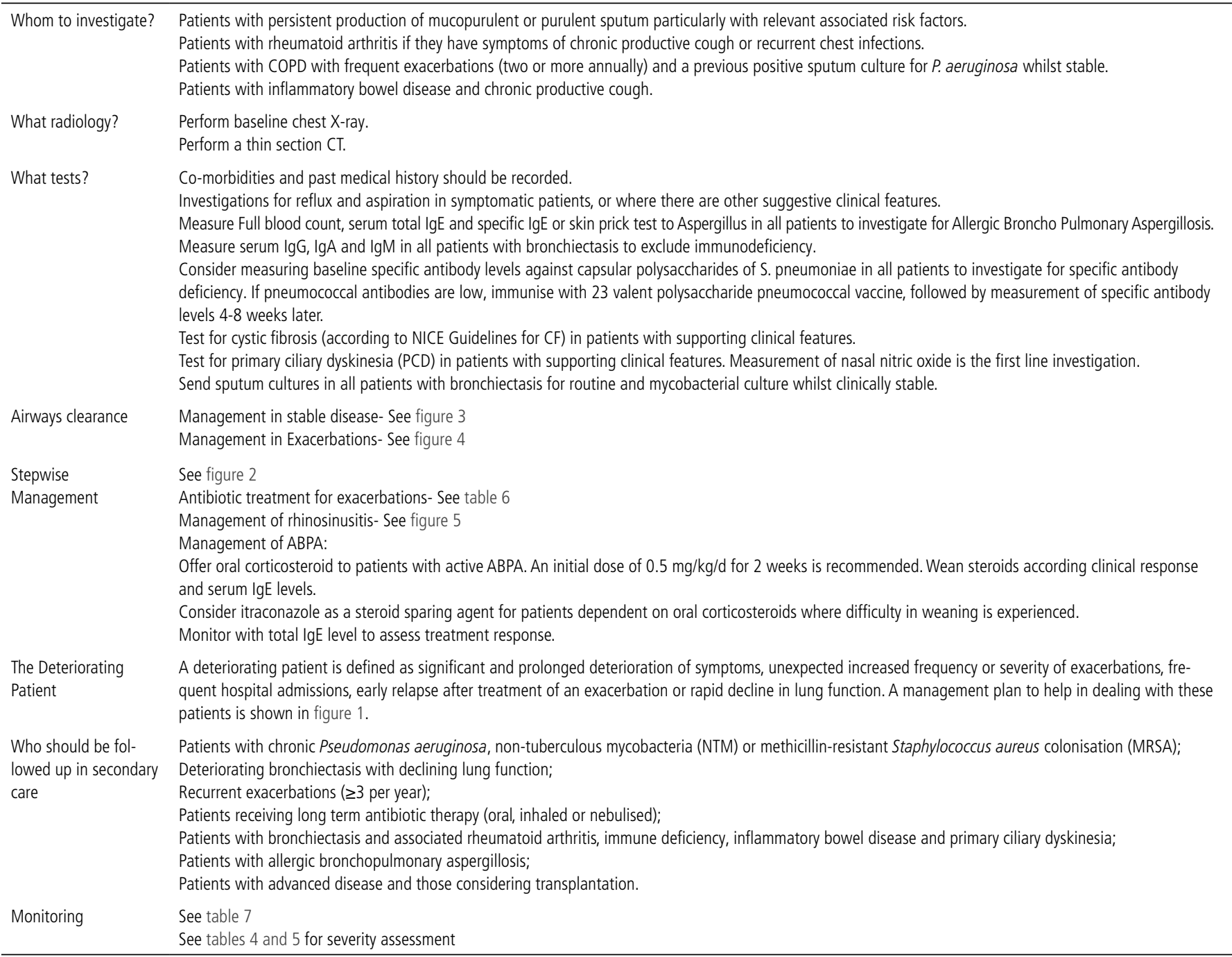




\section{SECTION 1}

\section{Introduction}

The BTS Guideline for non-CF Bronchiectasis was published in 2010. At the time of publication it was agreed by the Guideline Group and the BTS Standards of Care Committee that work on an update to the guideline should begin soon after publication to ensure that the guideline recommendations remained current in the light of new evidence.

Since the Guideline was published, BTS has produced Quality Standards for bronchiectasis in adults, and has offered an annual national audit, and from 2012, both adult and paediatric audit tools have been available. The BTS Standards of Care Committee approved a proposal to revise and update the guideline in 2013.

For guidance on treatment for patients with non-tuberculous mycobacteria and bronchiectasis please refer to the BTS Guideline on the management of non-tuberculous mycobacterial pulmonary disease. ${ }^{1}$

The guideline covers adult bronchiectasis. CF bronchiectasis is excluded from the scope of the guideline. Paediatric bronchiectasis was also excluded due to the difference in aetiology and approach in this group.

\section{Target audience for the guideline}

This guideline is aimed at all healthcare practitioners who are involved in the care of patients with bronchiectasis: this will include primary care clinicians (GPs, practice and district nurses), and hospital specialist teams in infectious disease, respiratory medicine (respiratory nurse specialists, respiratory physiotherapists, pharmacists, physicians and surgeons), microbiologists, and radiologists.

\section{Groups covered}

Adults

\section{Groups not covered}

The following patient groups and areas are excluded:

Patients with CF-bronchiectasis

Children up to and including 15 years old

Scope of the guideline

The guideline covers:

- Introduction section on prevalence;

- Diagnosis of bronchiectasis;

- Causes;

- Investigations and management in particular for CF, ABPA, common variable immune deficiency, NTM, coexistent asthma, COPD, ILD.

- A stepwise management plan which sets out investigation and treatment of bronchiectasis patients according to severity

- Definitions of severity of disease

\section{Areas not covered by the guideline}

Cystic Fibrosis, Non-tuberculous mycobacterial pulmonary disease

\section{Definition}

This guideline refers to the investigation and management of patients with symptoms of persistent or recurrent bronchial sepsis related to irreversibly damaged and dilated bronchi, namely clinical bronchiectasis. It does not cover the management of cystic fibrosis (CF) and, for the purposes of the guideline, 'bronchiectasis' is synonymous with the term 'non-CF bronchiectasis'. Likewise, it does not focus on traction bronchiectasis secondary to other lung pathologies, particularly the interstitial lung diseases, where bronchiectasis is commonly asymptomatic.

\section{SECTION 2 \\ Methodology}

This guideline is based on the best available evidence. The methodology used to write the guideline adheres strictly to the criteria as set by the AGREE collaboration, which is available online www.agreetrust.org/resource-centre/agree-ii/. The British Thoracic Society Standards of Care Committee guideline production manual is available at http://www.brit-thoracic.org. uk/guidelines-and-quality-standards/.

\section{Clinical questions and literature search}

Clinical questions were structured in the PICO (Patient, Intervention, Control, Outcome) format, (online appendix 1) to define the scope of the guideline and inform the literature search.

Systematic electronic database searches were conducted in order to identify potentially relevant studies for inclusion in the guideline. For each topic area the following databases were searched: Ovid MEDLINE (including MEDLINE In Process), Ovid EMBASE, and the Cochrane Library (including the Cochrane Database of Systematic Reviews, the Database of Abstracts of Reviews of Effects) from 1980.

The searches were first run in June 2014 and updated in June 2016 (see online appendix 2 for search strategy). Searches included a combination of indexed terms and free text terms and were limited to English language publications only. The initial search identified 3848 potential abstracts and the second search 1021 abstracts.

\section{Appraisal of literature}

Appraisal was performed to be compliant with the AGREE collaboration. Three individuals (AH, AS, ML) read the title and abstract of each article retrieved by the literature searches and decided whether the paper was definitely relevant, possibly relevant or not relevant to the project. Criteria formulated for categorising the abstracts into these three groups were:

- Whether the study addressed the clinical question.

- Whether the appropriate study type was used to produce the best evidence to answer the clinical question.

- Review articles were excluded.

- Abstract was in English.

- Abstracts were not rejected on the basis of the journal of publication, country in which the research was performed or published nor the date of publication.

The screened abstracts were allocated to the relevant section(s) of the guideline and two group members allocated to each guideline section. The full paper was obtained for all relevant or possibly relevant abstracts.

The first screening process identified 1022 of the initial 3848 reference abstracts to be definitely or possibly relevant to the guideline. Two guideline reviewers per section independently reviewed the abstracts to identify papers to be appraised for the guideline. The two reviewers for each section then independently appraised each paper assigned to them using the Scottish Intercollegiate Guidelines Network (SIGN) critical appraisal checklists. The reliability of the evidence in each individual study was graded using the SIGN critical appraisal check lists and is 


\begin{tabular}{|c|c|}
\hline Grade & Evidence \\
\hline $1++$ & $\begin{array}{l}\text { High quality meta-analyses, systematic reviews of RCTs, or RCTs with a } \\
\text { very low risk of bias }\end{array}$ \\
\hline $1+$ & $\begin{array}{l}\text { Well conducted meta-analyses, systematic reviews of RCTs, or RCTS with } \\
\text { a low risk of bias }\end{array}$ \\
\hline $1-$ & Meta-analyses, systematic reviews of RCTs, or RCTs with a high risk of bias \\
\hline $2++$ & $\begin{array}{l}\text { High quality systematic reviews of case-control or cohort studies or high } \\
\text { quality case-control or cohort studies with a very low risk of confounding, } \\
\text { bias or chance and a high probability that the relationship is casual }\end{array}$ \\
\hline $2+$ & $\begin{array}{l}\text { Well conducted case-control or cohort studies with a low risk of } \\
\text { confounding, bias or chance and a moderate probability that the } \\
\text { relationship is casual }\end{array}$ \\
\hline $2-$ & $\begin{array}{l}\text { Case-control or cohort studies with a high risk of confounding, bias or } \\
\text { chance and a significant risk that the relationship is not casual }\end{array}$ \\
\hline 3 & Non analytic studies, for example, case reports, case series \\
\hline 4 & Expert opinion \\
\hline
\end{tabular}

shown in the evidence tables $(++,+$ or -$)$. The body of evidence for each recommendation was summarised into evidence statements and graded using the SIGN grading system (see table 2).

Disagreements were resolved by discussion with the section partner. The second literature search in June 2016 yielded 1021 abstracts. Of these, 277 were identified as definitely or possibly relevant to the guideline. However, of the 277 identified, all relevant abstracts from this search had been identified by the Guideline Development Group (GDG) in the intervening time and incorporated.

\section{Considered judgement and grading of evidence}

The Guideline Development Group used the evidence tables to judge the body of evidence and grade recommendations for this guideline. Evidence tables (web appendix 3) are available online. Where evidence was lacking to answer the formulated clinical questions, expert opinions were obtained through consensus. The following were considered in grading of the recommendations:

The available volume of the body of evidence.

How applicable the obtained evidence was in making recommendations for the defined target audience of this guideline. Whether the evidence was generalisable to the target population for the guideline.

Whether there was a clear consistency in the evidence obtained to support recommendations.

What the implications of recommendations would be on clinical practice in terms of resources and skilled expertise.

Cost-effectiveness was not reviewed in detail as in-depth economic analysis of recommendations falls beyond the scope of this guideline.

Recommendations were graded from A to D as indicated by the strength of the evidence as shown in table 3. In line with SIGN guidance, 'minus' evidence was considered in context but in the absence of other 'plus' supporting evidence, it was discussed among the GDG regarding that point and any recommendation hence made was Grade D. Important practical points lacking any research evidence, nor likely to be research evidence in the future were highlighted as 'Good Practice Points' (GPP).

Research recommendations are also provided and the overall research questions are presented in PICO format in appendix 8 .

\begin{tabular}{|c|c|}
\hline Grade & Type of evidence \\
\hline$A$ & $\begin{array}{l}\text { At least one meta-analysis, systematic review, or RCT rated as } \\
1++ \text { and directly applicable to the target population or } \\
\text { A systematic review of RCTs or a body of evidence consisting principally } \\
\text { of studies rated as 1+directly applicable to the target population and } \\
\text { demonstrating overall consistency of results }\end{array}$ \\
\hline B & $\begin{array}{l}\text { A body of evidence including studies rated as } 2++ \text { directly applicable to the } \\
\text { target population and demonstrating overall consistency of results or } \\
\text { Extrapolated evidence from studies rated as } 1++ \text { or } 1+\end{array}$ \\
\hline C & $\begin{array}{l}\text { A body of evidence including studies rated as } 2+\text { directly applicable to the } \\
\text { target population and demonstrating overall consistency of results or } \\
\text { Extrapolated evidence from studies rated as } 2++\end{array}$ \\
\hline D & $\begin{array}{l}\text { Evidence of level } 3 \text { or four or } \\
\text { Extrapolated evidence from studies rates as } 2+\end{array}$ \\
\hline$\sqrt{ }$ & $\begin{array}{l}\text { Important practical points for which there is no research evidence, nor is } \\
\text { there likely to be any research evidence. The guideline committee wishes to } \\
\text { emphasise these Good Practice Points }\end{array}$ \\
\hline
\end{tabular}

\section{Drafting the guideline}

The Guideline Development Group corresponded regularly by email and meetings of the co-chairs sub group and full group were held in January, May and June 2014, January, May, September and December 2015, January, April, December 2016, January and November 2017. A number of teleconferences were also held. The BTS Standards of Care Committee (SOCC) reviewed the draft guideline in November 2017. The draft guideline was made available on-line March - April 2018 for public consultation and circulated to all the relevant stakeholders. The BTS SOCC re-reviewed the revised draft guideline in June 2018 and final SOCC approval granted in July 2018.

This BTS Guideline will be reviewed within 5 years from the publication date.

\section{Guideline group members and declarations of interest}

All members of the Guideline Group made declarations of interest in line with the BTS Policy and further details can be obtained on request from BTS. Guideline Development Group members are listed in appendix 1.

\section{Stakeholders}

The following organisations contributed to the consultation exercise:

ACPRC, ARNS, ARTP, BGS, BSTI, Edge Hill University, PCRSUK, ProAxsis Ltd, RCN, RCP Edinburgh, Trudell Medical.

\section{SECTION 3}

\section{How common is bronchiectasis?}

UK data in 2013 revealed the prevalence in women was $566 / 100000$ and in men 486/100 $000^{4}$.

Data from 12 US states over the period 1993-2006 demonstrate an average annual age-adjusted hospitalisation rate of 16.5 hospitalisations per 100000 population. Women and those aged over 60 years had the highest rate of hospitalisations. ${ }^{2}$ New Zealand hospital admission rates are reported as 25.7 per $100000^{3}$ highest in childhood and the elderly, and related to sex, socioeconomic deprivation and race.

Regarding co-morbidity coding that may provide information on aetiology, the UK data found no significant co-morbidity in 
$34 \%$ of patients, and the most common coded co-morbidities to be asthma (42\%) and COPD (36\%). ${ }^{4} \mathrm{HIV}$ was coded in $7 \%$, rheumatoid arthritis in $6 \%$, other connective tissue disease in $5 \%$, inflammatory bowel disease in $3 \%$ and antibody deficiency in $1 \%$.

\section{SECTION 4}

\section{How should the diagnosis of bronchiectasis be determined? Imaging}

There are no randomised controlled trials comparing different imaging techniques in establishing a diagnosis of bronchiectasis but there are several cohort studies of low to moderate quality as well as case series. Compared with HRCT sensitivity of CXR was $87.8 \%$ and specificity $74.4 \% .^{5}$ Compared with bronchography, thin section CT performed with $10 \mathrm{~mm}$ interspaces demonstrated a high accuracy in diagnosing bronchiectasis with false positive and negative rates for CT were $1 \%$ and $2 \%$ respectively. ${ }^{6}$ Volumetric CT has improved sensitivity and interobserver agreement compared with incremental/interspaced thin slice CT. ${ }^{7}$ Bronchiectasis was identified more commonly in helical than incremental thin slice images. Interobserver agreement was significantly better on a per segment basis with helical CT (kappa 0.87) than incremental (kappa 0.71), but radiation dose of helical CT was 3.4 times higher. Multidetector CT (MDCT) yielded higher sensitivity and specificity for the detection of bronchiectasis. Using MDCT as the reference standard and analysing data on a per lobe basis the sensitivity, specificity, positive and negative predictive values for incremental HRCT were $71 \%, 93 \%, 88 \%$ and $81 \%$ respectively. Interobserver agreement was also higher for MDCT. ${ }^{9}$

While the dose using volumetric CT using the same parameters is increased in comparison to incremental/interspaced images modifications of technique enable improved diagnostic accuracy with similar radiation dose. $^{7-9}$ Jung et al concluded that low dose CT at $40 \mathrm{mAs}$ provides more diagnostic information in the evaluation of bronchiectasis than incremental HRCT. ${ }^{10}$

While clearance of inhaled radiolabeled tracers from the lung is impaired in bronchiectasis this is non-specific and seen in other airways disease so cannot be considered diagnostic of bronchiectasis. Chronic bronchitis, bronchiectasis and asthma were all associated with slower clearance of inhaled radiolabeled tracers than healthy non-smokers $(\mathrm{P}<0.005)$ with overlap between these disease groups. ${ }^{11}$

Currie et al assessed radio-aerosol tracheobronchial clearance in first 6 hours in bronchiectasis (12 patients), COPD with sputum (7), COPD without sputum (8) and healthy controls. ${ }^{12}$ In bronchiectasis tracheobronchial clearance of inhaled radiolabeled tracers was significantly lower than controls but similar to COPD patients.

Ashford et al showed that ventilation scintigraphy with $99 \mathrm{mTc}$ DTPA in 20 patients with suspected bronchiectasis had low sensitivity for bronchiectasis (56\%) using bronchography as the reference standard. ${ }^{13}$

\section{Evidence statements}

Chest radiography (CXR) has limited sensitivity and specificity in diagnosing bronchiectasis particularly in mild disease $(2+)$.

Compared with bronchography thin section CT performed with $10 \mathrm{~mm}$ interspaces has a high accuracy in diagnosing bronchiectasis. $(2+)$

Volumetric CT has improved sensitivity and interobserver agreement compared with incremental/interspaced thin slice CT. $(2+)$
Using modifications of technique radiation dose of volumetric CT can be reduced to comparable levels to incremental imaging while providing higher accuracy. $(2+)$

While clearance of inhaled radiolabeled tracers from the lung is impaired in bronchiectasis this is non-specific and seen in other airways disease so cannot be considered diagnostic of bronchiectasis. (2-)

\section{Recommendations - Imaging}

> Perform baseline chest X-ray in patients with suspected bronchiectasis. (D)

> Perform a thin section CT to confirm a diagnosis of bronchiectasis when clinically suspected. (C)

> Perform baseline imaging during clinically stable disease as this is optimal for diagnostic and serial comparison purposes. (D)

\section{Good practice points \\ $\mathrm{CT}$ imaging protocol}

$\checkmark \quad$ The most appropriate imaging protocol will vary according to scanner technology and patient factors.

$\checkmark$ When using volumetric CT, dose reduction techniques including adaptive $\mathrm{mA}$ and $\mathrm{kV}$ and iterative reconstruction should be utilised where available.

$\checkmark$ Typical CT imaging parameters for the diagnosis of bronchiectasis are:

o Slice thickness: $\leq 1 \mathrm{~mm}$

o Reconstruction algorithm: - high spatial frequency

o kVp: $100-140$

o mAs (or effective mAs): 100-200

o Gantry rotation time: $<0.5 \mathrm{~s}$

\section{CT features of bronchiectasis}

$\checkmark$ Bronchiectasis is defined by bronchial dilatation as suggested by one or more of the following:

o Bronchoarterial ratio >1(internal airway lumen vs adjacent pulmonary artery)

o Lack of tapering

- Airway visibility within $1 \mathrm{~cm}$ of costal pleural surface or touching mediastinal pleura.

$\checkmark$ The following indirect signs are commonly associated with bronchiectasis:

o Bronchial wall thickening

o Mucus impaction

- Mosaic perfusion/air trapping on expiratory CT

\section{General}

$\checkmark$ CT scanning can also aid in identifying an aetiology of bronchiectasis for example, ABPA, NTM, primary ciliary dyskinesia, alpha one antitrypsin deficiency, Williams Campbell syndrome and a foreign body.

\section{SECTION 5}

\section{In whom should the diagnosis of bronchiectasis be suspected?}

The most common symptom in bronchiectasis is cough particularly with sputum production. ${ }^{14} 15$

Appendices 5 and 6 show the causes identified from international studies. Often no cause is found despite aetiological testing. Past infection (such as measles, whooping cough, pneumonia or tuberculosis) and is a possible cause of bronchiectasis, particularly if persistent symptoms develop soon after the infection. 


\section{Specific disease groups with associated bronchiectasis COPD}

A meta-analysis of 6 observational studies found that the prevalence of bronchiectasis was 54.3\% (range 25.6\%-69\%), more common in males (OR 1.62, 95\% CI 1.15 to 2.28) and with a greater smoking history (weighted mean difference 4.63 pack years, 95\% CI 1.61 to 7.65 pack years). Other features that distinguished patients with COPD and bronchiectasis from COPD alone included greater daily sputum production, higher exacerbation frequency, worse lung function, higher levels of inflammatory biomarkers, increased colonisation by potential pathogenic micro-organism (PPM) and increased rate of Pseudomonas aeruginosa (P. aeruginosa) colonisation. ${ }^{16}$ A systematic review and meta-analysis of 14 observational studies in COPD patients found that the presence of bronchiectasis was associated with worse airflow obstruction, isolation of PPM, increased risk of exacerbation and of mortality. ${ }^{17}$

\section{Alpha-1 antitrypsin deficiency (A1AT)}

A retrospective cohort study between 1995 and 2002 of 74 patients with PiZZ deficiency (mean age 50.6, SD 9.2 years) found radiological evidence of bronchiectasis in $94.5 \% .^{18}$ Another retrospective cohort study of 26 Irish patients with A1AT deficiency found that 14 had bronchiectasis, all of whom had PiZZ phenotype. ${ }^{19}$

\section{Asthma}

A careful cross-sectional analysis of 85 patients in secondary care with bronchiectasis found asthma in $27 \%$ of the clinic population while prevalence of asthma in the general population was $7 \% .{ }^{20}$ A large study of patients with difficult asthma found bronchiectasis on CT scan in $40 \%$ of selected patients; the criteria for scanning were not stated but the scanned patients were older, with a longer duration of disease, on more corticosteroid treatment and with poorer lung function and more neutrophilic airway inflammation on sputum cytology than those who were not scanned. ${ }^{21}$ In a case-control study matching patients with steroid-dependent asthma to those managed without regular oral corticosteroids, it was noted that bronchiectasis was much more common in the former group and the overall prevalence of otherwise unexplained bronchiectasis was $12 \%$ across both groups, rising to $20 \%$ in the steroid-dependent group. ${ }^{22}$

\section{Rhinosinusitis}

Rhinosinusitis is common in bronchiectasis patients, ${ }^{23}$ but only one study appears to have assessed the prevalence of bronchiectasis in chronic sinusitis, finding it in 3 out of 60 patients $(5 \%) .^{24}$

\section{Chronic systemic infection}

Chronic infection such as HIV or HTLV-1 appears to increase the risk of bronchiectasis. ${ }^{25} 262728$

\section{Rheumatoid arthritis}

Clinical studies of patients with rheumatoid arthritis (RA) have found varying rates of bronchiectasis on CT scan ranging from $4 \%$ to $58 \%$. $^{29-38}$

The diagnosis of bronchiectasis may occur in early or established RA, and presentation and diagnosis with bronchiectasis may pre-date the diagnosis of even seropositive RA. Significantly more erosive changes were observed on hand and foot radiology in 53 patients with bronchiectasis and RA versus 50 patients with RA alone, and both rheumatoid factor (RF) and anti-cyclic citrullinated peptide (anti-CCP) antibodies were higher in those with bronchiectasis. ${ }^{39}$ In that study, bronchiectasis preceded the onset of RA in $58 \%$. $^{39}$

A large study on patients hospitalised with RA established the frequency of appropriate symptoms first and then investigated with CT scan. Out of 453 patients questioned, 13 had symptoms suspicious for bronchiectasis and 9 of the 10 patients scanned had confirmed bronchiectasis, giving a prevalence of $2.9 \%$ of symptomatic bronchiectasis in this population. $^{40}$

\section{Other connective tissue diseases}

Bronchiectasis has been noted in other connective tissue diseases including primary Sjogren's syndrome, Marfan's syndrome, systemic sclerosis, systemic lupus erythematosus, and ankylosing spondylitis.

A study of a 507 patient cohort with primary Sjogren's syndrome (PSS) identified 120 patients with suspected pulmonary disease, and found bronchiectasis on CT scan in 50 patients. ${ }^{41}$ Retrospective studies confirm the association. ${ }^{42-44}$

A retrospective review of 79 patients with Marfan's who underwent HRCT imaging found evidence of bronchiectasis in $28 \%$. $^{45}$ Airway dilatation was described as not severe, often confined to one lobe and was said to localise with anatomical abnormalities such as pectus excavatum, although fibrosis due to previous tuberculosis was noted in some patients. ${ }^{45}$ Studies in ankylosing spondylitis have found incidence of bronchiectasis to range from $7.2 \%$ to $51.2 \% .^{46-51}$ In 7 of 34 patients with systemic lupus erythematosus (SLE) who prospectively underwent HRCT imaging, bronchiectasis was observed. ${ }^{52}$ A small study of systemic sclerosis found a high rate of bronchiectasis on CT in 13 (59.1\%) of 22 patients. ${ }^{53}$ In a study of scleroderma and pulmonary hypertension, bronchiectasis independent of interstitial lung disease (ILD) was found on CT scan in 6 of 44 patients with restrictive lung function or crackles. $^{54}$

\section{Inflammatory Bowel Disease}

There is a recognised association between bronchiectasis and inflammatory bowel disease (IBD). A prospective study of 95 patients with IBD (83 ulcerative colitis (UC) and 12 Crohn's disease (CD)) who underwent HRCT scans found evidence of bronchiectasis in 9, all of whom had UC and no reported respiratory symptoms. ${ }^{55}$ A cohort study of $30 \mathrm{UC}$ and $9 \mathrm{CD}$ patients undergoing CT scans found bronchiectasis in two patients. ${ }^{56}$ In 36 consecutive IBD patients (23 UC, 13 CD) studied for pulmonary disease, bronchiectasis was found on CT in three patients. $44 \%$ of the total study population had respiratory symptoms, but sputum production was described in only two patientsthe relationship between symptoms and CT findings was not described. ${ }^{57}$ A literature review found bronchiectasis in 44 out of 155 patients with inflammatory bowel disease. ${ }^{58}$

A retrospective case note review of 10 patients with IBD (5 UC, 5 CD) and bronchiectasis found that eight had developed respiratory symptoms only following surgery for their IBD. ${ }^{59}$ These patients had had IBD for a median of 15 (9-35) years and for those developing pulmonary symptoms following surgery, the time from surgery to symptoms ranged from 2 weeks to 30 
years. ${ }^{59}$ In a study of 17 IBD patients with respiratory symptoms, the diagnosis of IBD preceded the onset of their pulmonary symptoms in 16.13 of these patients were found to have bronchiectasis. $^{60}$

\section{Evidence statement}

Studies in healthy populations do not provide a strong body of evidence but suggest that persistent mucopurulent or purulent sputum production in the stable state is suspicious for underlying bronchiectasis, particularly if there is a past history of major respiratory infection (eg, measles, whooping cough, pneumonia, tuberculosis) or ongoing rhinosinusitis (2-).

There is a high frequency of bronchiectasis in patients with COPD $(2++)$, particularly with more severe airflow obstruction $(2++)$.

The presence of bronchiectasis in patients with COPD is typically associated with chronic productive cough, isolation of PPMs from sputum, particularly $P$. aeruginosa, increased airway inflammation, frequent or severe exacerbations or admissions to hospital for exacerbations $(2+)$.

Bronchiectasis is associated with alpha one antitrypsin deficiency, particularly with the phenotype PiZZ $(2+)$

Asthma is found in higher prevalence in patients with bronchiectasis than in the general population, $(2+)$, and bronchiectasis appears more common in asthma, particularly in difficult to treat disease (2-).

There is an association between rheumatoid arthritis and bronchiectasis $(2+)$. The diagnosis of bronchiectasis may precede the onset of rheumatoid arthritis (2-).

There is an association between bronchiectasis and other connective tissue diseases (2-).

There is an association between inflammatory bowel disease and bronchiectasis $(2+)$.

Bronchiectasis has been reported in patients with HIV infection at a frequency higher than in the general population (2-).

Bronchiectasis has been reported in patients with HTLV-1 infection with inflammatory complications at a frequency higher than in the general population. (3)

\section{Recommendations}

> Consider investigation for bronchiectasis in patients with persistent production of mucopurulent or purulent sputum particularly with relevant associated risk factors. (D)

> Consider investigation for bronchiectasis in patients with rheumatoid arthritis if they have symptoms of chronic productive cough or recurrent chest infections. (C)

$>$ Consider investigation for bronchiectasis in patients with COPD with frequent exacerbations (two or more annually) and a previous positive sputum culture for $P$. aeruginosa while stable. (B)

$>$ Consider investigation for bronchiectasis in patients with inflammatory bowel disease and chronic productive cough. (C)

\section{Good practice points}

$\checkmark$ In at risk groups, if bronchiectasis is suspected, bronchiectasis needs confirmation.

$\checkmark \quad$ In patients with COPD, investigation for bronchiectasis may be appropriate especially in the presence of chronic productive cough with positive sputum cultures for PPM while stable or two or more exacerbations in the preceding 12 months. $\checkmark \quad$ In patients with asthma, investigation for bronchiectasis may be appropriate with severe or poorly-controlled disease.

$\checkmark \quad$ In patients with a history of HIV-1 infection, solid organ and bone marrow transplant, and history of immunosuppressive therapy for lymphoma and vasculitis, investigation for bronchiectasis may be appropriate with symptoms of chronic productive cough or recurrent chest infections.

$\checkmark$ In patients with chronic rhinosinusitis, investigation for bronchiectasis may be appropriate with symptoms of chronic productive cough or recurrent chest infections.

$\checkmark$ In patients with other connective tissue disease or inflammatory bowel disease, investigation for bronchiectasis may be appropriate if they have symptoms such as chronic productive cough or recurrent chest infections.

$\checkmark$ Investigation for bronchiectasis may be appropriate in otherwise healthy individuals with a cough that persists for longer than 8 weeks, especially with sputum production or a history of an appropriate trigger (see BTS Recommendations for the management of cough in adults ${ }^{61}$ ).

\section{SECTION 6}

\section{Investigations for causes of bronchiectasis}

\section{Introduction}

Single centre studies have shown that investigations into the underlying cause of bronchiectasis can change patient management in a significant proportion of cases (5\%-37\%) and identify previously unrecognised conditions such as allergic bronchopulmonary aspergillosis (ABPA), primary antibody deficiency syndromes and cystic fibrosis (CF) which have important therapeutic and prognostic implications. ${ }^{23}{ }^{62-66}$ Early studies were conducted in specialist centres but more recently studies of adult patients have been conducted in general chest clinics; online appendix 5 summarises those most relevant to the UK population and includes a systematic review of 56 studies covering 8608 patients from across the world. ${ }^{67}$

Review of imaging can suggest possible aetiologies although the reliability of this approach has not been formally assessed. For example disease in a single lobe might be due to obstruction from tumour or foreign body, and bronchoscopy would be an appropriate investigation in such cases. Post-tubercular disease might be supported by distribution or the presence of calcification. A diagnosis of post-infective disease would be supported by onset of symptoms soon after the illness. Bronchiectasis following measles or whooping cough is often bilateral and lower lobe.

It can be difficult to ascertain if conditions such as COPD, rheumatoid arthritis, inflammatory bowel disease or GORD are causative of bronchiectasis. Even with an obvious apparent aetiology, there may be value in carrying out standard investigations at baseline, since patients with established disease may later develop an immunodeficiency or ABPA.

\section{Standard laboratory tests}

The evidence for the role of aetiological investigations is derived from studies of low to moderate quality; other significant limitations include a lack of standardised diagnostic testing panels for bronchiectasis, resulting in marked variation in the performance of some diagnostic assays (CF mutation analysis, test immunisation of vaccine responses), use of different technological platforms for some diagnostic assays which may give rise to discrepancy in test results (measurement of pneumococcal and aspergillus IgG antibodies) and variation in the use of reference intervals to define presence or absence of disease even when 
using same or very similar diagnostic tests (Aspergillus blood IgE levels).

Although most individual studies are of low/moderate quality, the overall findings indicate that antibody deficiency syndromes and ABPA should be investigated in all newly presenting patients with bronchiectasis (see appendix 5).

\section{Tests for specific disease groups with associated bronchiectasis}

\section{Allergic bronchopulmonary aspergillosis}

Data suggests that this is a common cause of bronchiectasis in the UK, between $1 \%$ and $11 \%$ in UK series. ${ }^{23} 62636869$

The diagnostic criteria for ABPA are well established for asthma and have been modified for cystic fibrosis. ${ }^{70}$ They include a history of asthma, evidence of Aspergillus fumigatus ( $A$. fumigatus) IgE sensitisation on blood and/or skin tests, elevated total IgE levels and eosinophil counts, detection of Aspergillus precipitins or IgG, isolation of A. fumigatus in sputum cultures, presence of pulmonary infiltrates on chest $\mathrm{x}$ rays and distribution of bronchiectasis on CT chest scans. ${ }^{71-73}$ The presence of high attenuation mucus on CT scan may be useful in diagnosis and in staging the severity of ABPA. ${ }^{74} \mathrm{ABPA}$ is an aetiology for bronchiectasis, and may be a complicating factor in established disease. Inconsistent diagnostic criteria have been used in the previously cited studies of aetiology of bronchiectasis. ${ }^{23} 62-657576$ There are no studies in patients presenting with bronchiectasis that can help to establish the optimal number of investigations and the most important criteria for a diagnosis of ABPA in this context; criteria for asthma are generally used.

Sensitisation to A. fumigatus can be detected using blood specific IgE or skin tests however there is limited evidence on which method is best. Intradermal skin tests are more sensitive than standard skin prick to detect Aspergillus-specific IgE ${ }^{77}$ but interpretation can be difficult and they are most used in academic centres with a specialist interest. A recent single centre specialist care study from India which used latent class analysis (the statistical test used to assess diagnostic test performance in conditions without a diagnostic gold standard) showed that detection of Aspergillus-specificIgE in blood was more sensitive than intradermal skin tests for the diagnosis of ABPA. ${ }^{78}$

Diagnostic criteria for ABPA include positive IgE blood and cutaneous skin test results as there may be a small proportion of patients who have discrepant blood and skin test results. ${ }^{73}$ Use of blood rather than cutaneous skin test has the advantage that patients on anti-histamines do not need to stop treatment, and quality data on laboratory performance is more widely available than on skin testing. IgE sensitisation to A. fumigatus has been reported in $19 \%$ of unselected patients presenting with bronchiectasis using blood tests ${ }^{62}$ however the optimal concentration required to diagnose $\mathrm{ABPA}$ in bronchiectasis cohorts has not been established. ${ }^{236264}$

Similarly the use and definition of raised total IgE blood tests in diagnosis of ABPA has been variable in bronchiectasis cohorts. $^{23}$ 62-64 The earliest available total IgE test should be used, as the total IgE concentration declines significantly with remission of ABPA on oral corticosteroids whereas no consistent effects on Aspergillus-specific IgE occur. ${ }^{79}$ There is no consensus on what value of $\operatorname{IgE}$ is required for diagnosis of ABPA. In a single specialist centre study the sensitivity of an IgE concentration more than $1000 \mathrm{IU} / \mathrm{ml}$ for diagnosis of ABPA in asthmatic patients was $97.5 \% .^{78} \mathrm{~A}$ normal IgE level in a corticosteroid naïve patient with bronchiectasis means that ABPA is highly unlikely. A combination of testing for Aspergillus-specific IgE and total IgE has been proposed as the most sensitive way to screen for ABPA in patients with asthma, with secondary more specific tests to confirm the diagnosis ${ }^{78}$; this approach has not been validated in patients with bronchiectasis.

An eosinophil count above $1.0 \times 10^{9} / \mathrm{L}$ is a major criterion for $\mathrm{ABPA}^{78}$ but should be used as a second line test as normal counts do not exclude this disease ${ }^{80}$ and there is no significant difference in eosinophil counts between patients with A.fumigatus sensitisation and those with ABPA. ${ }^{81}$ Previous studies in asthmatic patients have all been conducted in a single centre and have relied on manual eosinophil counts which are less accurate than automated diagnostic platforms. ${ }^{78-81}$

Aspergillus-specific IgG antibodies can be found in 70\%-90\% of patients with ABPA using agar gel immune diffusion technology. ${ }^{8283}$ The results of these studies may not be applicable to current epidemiology of A. fumigatus ABPA, as a recent study showed that the diagnostic performance of the Ouchterlony diffusion technique to detect IgG antibodies had declined and Aspergillus precipitins were only seen in $43 \%$ of patients with ABPA. ${ }^{78}$ In addition immunoprecipitation techniques to detect Aspergillus-specific IgG antibodies are not widely available in diagnostic UK laboratories, having been replaced initially by counter immuno-electrophoresis (CIE) initially and more recently by ELISA. Only one study of aetiology in bronchiectasis $^{63}$ defined criteria for a positive Aspergillus precipitins test result and diagnostic techniques used to determine presence of Aspergillus-specific IgG antibodies were not described by any investigators. A small UK study comparing the diagnostic test of CIE and ELISA in patients with Aspergillus related lung disorders showed that the sensitivity of ELISA (41\%-46\%) for ABPA was significantly greater than CIE (15\%) however lack of relevant disease control group and absence of data of current anti-fungal therapy make it difficult to ascertain the clinical value of Aspergillus-specific IgG detection using current diagnostic techniques. ${ }^{84}$ Aspergillus precipitins can be raised in other conditions such as aspergilloma and chronic pulmonary aspergillosis and thus the more likely diagnostic role if any for Aspergillus-specific IgG antibodies will be as a second line confirmatory test.

Culture of A. fumigatus from lung secretions is supportive but not diagnostic of ABPA as this fungus can be isolated in a number of other diseases. Rate of positive sputum cultures in historical cases of ABPA ranges from $63 \%-97 \%,{ }^{82} 83$ however in the only study of aetiology of bronchiectasis in which sputum cultures were part of the standard diagnostic panel, $A$. fumigatus was only isolated in $9 \%$ of cases of ABPA. ${ }^{62}$ Reasons for lower yields of A. fumigatus in sputum cultures could include exposure to azole therapy and differences in sputum processing and culturing technologies in microbiology laboratory services. ${ }^{85}$

In the absence of stronger evidence, all the following criteria based on expert consensus are suggested for steroid naïve patients.

o Associated clinical deterioration (increased cough, wheeze, increased sputum production, worsening lung function, exacerbations)

o Total serum IgE level $>500 \mathrm{IU} / \mathrm{mL}$ (or $\mathrm{ku} / \mathrm{L}$ )- precise cut-off is not known.

o Immediate cutaneous reaction to Aspergillus (skin prick test weal of $>3 \mathrm{~mm}$ in diameter with surrounding erythema, patient not on systemic antihistamines at the time). 
- Increased Aspergillus species specific IgE antibodies

o New radiological infiltrates and/or high attenuation mucus plugging. In later stages, proximal bronchiectasis develops.

Supportive findings include peripheral blood eosinophilia, and Aspergillus precipitins.

\section{Immune dysfunction}

Immune deficiency occurred in $5.8 \%$ of patients in a multicentre cross sectional European study ${ }^{63}$ and in $5 \%$ of patients in a large systematic review of aetiological studies. ${ }^{67}$ In adults, almost all cases of immune deficiency are likely to involve defects in antibody production. Recognition of significant antibody syndromes such as common variable immune deficiency (CVID) is important as immunoglobulin replacement therapy can reduce exacerbation frequency and improve clinical outcomes. ${ }^{86}$ In recent years there has been increasing recognition that treatment for haematological malignancies is associated with increased risk of antibody deficiency syndromes and bronchiectasis ${ }^{65}$ which may respond well to immunoglobulin replacement. ${ }^{87}$

\section{Serum immunoglobulins ( $\lg G, \lg \mathrm{A}, \& \lg \mathrm{M})$ and electrophoresis}

A polyclonal rise in serum $\operatorname{IgG}$ and IgA is commonly seen in patients with bronchiectasis and reflects chronic infection and inflammation. ${ }^{64}$ There are no studies addressing the clinical utility of serum electrophoresis in the investigation of bronchiectasis, but this test is essential to determine whether raised serum immunoglobulins are polyclonal or monoclonal. The presence of a monoclonal immunoglobulin in serum needs further investigation to diagnose myeloma, Waldenstrom's macroglobulinaema, chronic lymphatic leukaemia and monoclonal gammopathy of uncertain significance (MGUS). The prevalence of MGUS is $3.2 \%$ in individuals over the age of 50 with increased rates in males, older age and African Americans. ${ }^{88} \mathrm{~B}$ cell lymphoproliferative disorder and MGUS are associated with increased rates of bacterial chest infections ${ }^{89}$ and patients need appropriate follow-up to monitor for haematological malignancy. Antibody deficiency secondary to B cell lymphoproliferative disorders has been found in a number of studies examining aetiology of bronchiectasis, ${ }^{23} 65$ although the presence or absence of immunoglobulin monoclonal proteins was not stated.

Isolated reduction in IgG, IgA and IgM is not uncommon ${ }^{62647690}$ and will usually require further evaluation to assess clinical significance in management of bronchiectasis. Minor reduction in IgG and IgM can be secondary to steroid therapy, B cell lymphoproliferative disease or unclassified antibody deficiency syndromes, which may require further specialised investigation (assessment of vaccine responses, lymphocyte immune phenotyping and enumeration of lymphocyte subsets) and/or involvement of other medical specialists to manage relevant co-morbidity.

\section{Full blood count (FBC)}

There are no studies examining the clinical utility of FBC in determining the cause of bronchiectasis but an abnormal profile may give important diagnostic clues. Persistent low neutrophil and lymphocyte counts in patients with stable disease may suggest an underlying secondary or primary immune deficiency. Lymphocytosis can be a marker of B cell lymphoproliferative disease and a raised eosinophil count may support a diagnosis of ABPA. Reduced platelet count can be a feature of primary and secondary immune deficiencies whereas thrombocytosis is associated with active RA and IBD.
Measurement of baseline specific antibody levels against capsular polysaccharides of Streptococcus pneumoniae

Measurement of functional antibody responses to T cell dependent protein or glycoprotein antigens and $\mathrm{T}$ cell independent polysaccharide antigens is an essential criterion for diagnosis of primary antibody deficiency syndromes including CVID and specific polysaccharide antibody deficiency (SAD). ${ }^{91} 92$ Assessment of functional antibody responses is also indicated to assess clinical significance of partial/total IgA deficiency, isolated reductions in IgG and IgM, reduced IgG and IgM and reduced immunoglobulin isotypes in patients with $\mathrm{B}$ cell lymphoproliferative disorders. In clinical practice, analysis of $\mathrm{T}$ cell independent antigen response is usually assessed by measurement of pneumococcal antibodies, which is also clinically relevant in patients with bronchiectasis.

The initial step to investigate functional antibody response is to measure pneumococcal antibody levels, and if low, to immunise with 23 valent carbohydrate Pneumococcal vaccine and check post vaccine level 4-8 weeks later. Antibody levels below protective threshold are deemed to be indicative of impaired vaccine responses and functional antibody deficiency.

The proportion of unselected bronchiectasis patients with reduced levels of pneumococcal antibodies varies between $2 \%-21 \%$. $^{23}{ }^{62-64}$ Differences in reported studies may relate to study setting, differences in pneumococcal antibody assays, or the pneumococcal antibody level at which test immunisation was considered. Some studies reporting pneumococcal antibody levels in unselected patients with bronchiectasis ${ }^{63-66}$ do not distinguish between the need to assess the functional significance of reduced serum immunoglobulins in patients with bronchiectasis and the use of this test solely to screen for patients with SAD.

The optimal pneumococcal level at which test immunisation should be considered varies: criteria for test immunisation have included patients with baseline pneumococcal antibody levels in the lowest fifth centile ${ }^{23}$ or $25^{\text {th }}$ centile ${ }^{62}$ for healthy controls. Studies using in-house assays to measure total $\operatorname{IgG}$ response to pneumococcal immunisation have reported the largest number of patients with low pneumococcal antibody and reduced pneumococcal vaccine responses. ${ }^{62} 93$ Pneumococcal in-house assays have largely been replaced by commercial assays which may not give equivalent results, resulting in the need to define pneumococcal antibody reference intervals and decision points at which pneumococcal test immunisation is offered. There is currently a move towards serotype based assays of individual pneumococcal serotypes, usually 12-14 in total ${ }^{929495}$; only a limited number of laboratories offer this test in the UK. In addition there is only moderate agreement between quoted protective levels based on gold standard ELISA and newer multiplex bead array diagnostic platforms and disagreement concerning use of protective antibody levels (invasive disease as opposed to mucosal infection).

Use of protein conjugate pneumococcal vaccine in childhood may alter dynamics of polysaccharide pneumococcal vaccination in adults. ${ }^{91}$ Bronchiectasis clinics and immunology laboratories need to have data on distribution of either total and/or individual serotype pneumococcal IgG levels in age- matched healthy controls and local agreement on the decision point used in pneumococcal antibody tests as to when patients with bronchiectasis are offered 23 valent polysaccharide vaccine test immunisation.

Reduced pneumococcal vaccine responses are part of the diagnostic criteria for a number of primary antibody deficiencies ${ }^{95}$ and have important implications for the use of immunoglobulin 
replacement therapy in patients with bronchiectasis with primary and secondary antibody deficiencies. There are no uniform criteria as to what constitutes a protective threshold antibody for total IgG pneumococcal (23 valent carbohydrate Pneumococcal vaccine) levels, however a post vaccine pneumococcal serotype concentration greater than $1.3 \mathrm{ug} / \mathrm{mL}$ is believed to offer protection against strain specific invasive pneumococcal disease. ${ }^{96}$ Expert opinion suggests that failure to generate a protective titre (1.3microG/ microL) to more than $70 \%$ serotypes post polysaccharide pneumococcal vaccination is indicative of moderate to severe functional antibody deficiency. ${ }^{92}$ These criteria have not been validated in clinical trials and it has been argued they may overestimate the number of patients with functional antibody deficiency.

The rate of impaired post pneumococcal test immunisation responses in patients with bronchiectasis has varied from $0 \%-20 \%,{ }^{23} 62-6593$ with the main sources of variation being the nature of the patient cohort, different methodology in assessing the response, and variations in the definition of low baseline levels and a normal response. Criteria used to define adequate pneumococcal vaccine responses have included results obtained from post vaccine serology from healthy controls, ${ }^{93}$ use of historical reference data ${ }^{62}$ or expert opinion, ${ }^{65}$ however a number of studies have not reported their criteria to select patients for test immunisation, the test methodology and the definition of normal pneumococcal vaccine response. ${ }^{23} 6466$

Interpretation of pneumococcal test immunisation results needs to take into account history of recent infections, previous vaccinations, use of immunosuppressive and cytotoxic chemotherapy and the finding that impaired pneumococcal test immunisation responses can be seen in up to $10 \%$ of healthy individuals. The increasing use of pneumococcal serotype assays for which there is no data for clinical utility in patients with bronchiectasis and limited agreement on what constitutes an adequate immune response ${ }^{91}$ suggest that there needs to be appropriate liaison between bronchiectasis clinics and diagnostic immunology services as to which patients should be offered test immunisation, what technology is used to measure pneumococcal antibodies, and what criteria are used to define a normal vaccine response.

Interpretation can be challenging and thus it is advised the results are discussed with an immunologist, as there has not been universal agreement about the degree of vaccine response to define specific antibody deficiency. If there is no local access to an immunologist, suggest discussion with a specialist bronchiectasis service that has an immunologist as part of the multidisciplinary team.

More specialised immunological investigations are described in the appendix; these would usually be carried out by immunologists or in close liaison with immunology services (see appendix 4).

\section{Rheumatoid arthritis}

Rheumatoid arthritis is the aetiology for a small proportion of patients referred to secondary care with bronchiectasis $\left(2 \%-5 \%{ }^{23} 6264\right)$. Given the strong association between rheumatoid arthritis and bronchiectasis, auto-antibodies such as rheumatoid factor (RF) and anti-cyclic citrullinated peptide (anti-CCP) antibodies have been considered as aetiological tests. Presence of circulating RF in patients was shown to be more prevalent in patients with bronchiectasis compared with healthy controls in a prospective multi-centre case-control observational study. ${ }^{97}$ However anti-CCP antibodies were strongly positive in only 12\% (4 patients) of those who were RF-positive. Two of those four patients subsequently developed rheumatoid arthritis in a 12 month follow-up period.

\section{Recurrent aspiration}

Recurrent aspiration due to dysphagia or gastro-oesophageal reflux is recognised to cause bronchiectasis, with a reported prevalence (based on the presence of symptoms) of between $1 \%$ and $11.3 \%$ in adults 2362646576 and a higher rate in a paediatric cohort attending a specialist referral centre. ${ }^{75}$ However, gastro-oesophageal reflux is common (and therefore can be mistakenly attributed as a causal factor), and can be asymptomatic (and therefore not considered). Limited case series are suggestive of a role for surgical management of reflux disease. ${ }^{98} 99$ Dysphagia can be caused by neurological disorders, oesophageal dysmotility and anatomical disorders (trachea-oesophageal fistula, previous surgery, pharyngeal pouch, late effects of head and neck radiotherapy). The likelihood of aspiration may be increased by vocal cord dysfunction. A careful gastrointestinal history focussing on symptoms of reflux and dysphagia should therefore be undertaken.

A number of different investigative modalities are available and include videofluoroscopic swallow study, upper gastrointestinal endoscopy, ambulatory oesophageal manometry and $\mathrm{pH}$ studies and flexible endoscopic evaluation of swallow. The sensitivity and specificity of these tests when used to assess aspiration in bronchiectatic patients have not been investigated. The method of evaluation should therefore be tailored according to clinical features.

\section{Cystic fibrosis}

Cystic fibrosis (CF) bronchiectasis is managed as a distinct clinical entity. Routine newborn screening for CF has been standard in the UK since 2007, but the disease may still present in later life and should be considered as a possible aetiology. CF is mainly seen in Caucasian populations and is rare in Asian populations. ${ }^{100}{ }^{101}$ Clinical features supportive of CF include an early onset, history of malabsorption, male infertility and childhood steatorrhoea. Radiologically, upper lobe bronchiectasis predominates and there is frequently a history of Staphylococcus aureus on sputum culture.

Standard investigative methods for CF include the sodium chloride sweat test and CFTR mutation analysis. There are established guidelines for the sweat test which should measure chloride concentration on two occasions. A negative test has $<30 \mathrm{mmol} / \mathrm{L}$ chloride, a borderline test $30-60 \mathrm{mmol} / \mathrm{L}$ and a positive test $>60 \mathrm{mmol} / \mathrm{L}$. ${ }^{102} 103$

The CFTR gene is located on chromosome seven and to date $>2000$ CFTR mutations have been identified (www.genet. sickkids.on.ca). It has been demonstrated that the greater the number of mutations screened, the greater the number of patients with CF will be identified. ${ }^{104}$ Advice from the local clinical genetics department should be sought. Ancillary tests such as nasal potential difference and assessments of pancreatic exocrine function offer additional evidence only and are not diagnostic in themselves. Their use should be limited to within specialist CF centres.

The incidence of $\mathrm{CF}$ as a cause in adults presenting with bronchiectasis ranges from $0.6 \%-2.7 \%$ (see online appendix 6).

For recommendations for the diagnosis and management of CF refer to the NICE CF Guidelines. ${ }^{105}$

\section{Ciliary dysfunction \\ Primary Ciliary Dyskinesia}

Primary ciliary dyskinesia is usually an autosomal recessive disorder causing ultrastructural defects in the cilia affecting normal motility. It is characterised by upper and lower 
respiratory tract infections, chronic sinopulmonary infection, development of bronchiectasis and loss of lung function. Male infertility is common and situs inversus may be present. Kartagener's syndrome describes the classic triad of situs inversus, bronchiectasis and chronic sinusitis due to a congenital reduction or absence of ciliary function.

PCD is typically diagnosed in the paediatric population. The incidence of PCD as a cause of bronchiectasis in adults ranges from $0.9 \%-10.3 \%$ (see online appendix 6). Investigative methods that have been used are either screening tests including measurement of nasal nitric oxide (nNO) and the saccharin taste test, or diagnostic tests assessing ciliary structure and function. The saccharin taste test is no longer recommended in the investigative process for PCD ${ }^{106} \mathrm{nNO}$ levels are abnormally low in PCD. ${ }^{107-109} \mathrm{~A}$ large prospective study found the positive predictive value (PPV) of $\mathrm{nNO}$ (using a cut-off value of $<30 \mathrm{~nL} / \mathrm{min}$ ) for a diagnosis of PCD to be 0.76 (CI 0.6 to 0.88 ) and the negative predictive value (NPV) 0.99(CI0.971.00 ) with a sensitivity of $91 \%$ and specificity of $96 \% .{ }^{110}$ In a separate study, the PPV of nNO was $87 \%$ and NPV $97 \%$ in discriminating between PCD and non PCD bronchiectasis. ${ }^{111}$

Nasal brushing is a simple, minimally invasive method to obtain ciliated epithelium. Analysis of ciliary beat frequency and pattern is usually undertaken using high speed video microscopy analysis (HSVMA). Normal ciliary beat frequency $(11-20 \mathrm{~Hz})$ and ciliary beat pattern is a qualitative assessment. The PPV of HSVMA for a diagnosis of PCD has been shown to be 0.61 (CI 0.5 to 0.7 ) and NPV 1.0 (CI 0.99 to 1.00 ) in a major UK centre's referral population, with a sensitivity of $100 \%$ and a specificity of $93 \% .{ }^{110}$ Transmission electron microscopy (TEM) to assess ciliary ultrastructure has been shown to have a PPV of 1.00 (CI0.94-1.00) and a NPV of 0.95 (CI 0.92 to 0.97) with a sensitivity of $79 \%$ and specificity of $100 \% .{ }^{110}$ Both HSVMA and TEM are excellent diagnostic tools but require expertise and specialist review. Further information is given in the ERS guidelines for the diagnosis of PCD. ${ }^{106}$

(Diagnostic centres for PCD testing in the UK are located in London (http://www.rbht.nhs.uk/patients/condition/primaryciliary-dyskinesia/) Leicester (http://www.leicestershospitals.nhs. uk/), and Southampton (http://www.uhs.nhs.uk/OurServices/ Childhealth/Primary-ciliary-dyskinesia/PCD-diagnostic-service. aspx).

\section{Young's syndrome}

The combination of primary azoospermia, chronic rhinosinusitis and bronchiectasis was first briefly described in 1970 as the Berry-Perkins-Young Syndrome. ${ }^{12}$ Further studies suggested that this consisted of a specific form of obstructive azoospermia while the airway features included poor mucociliary clearance. Crossover with cystic fibrosis was ruled out ${ }^{113}$ and in general no specific ciliary beat frequency or ciliary ultrastructural abnormalities were identified, ${ }^{114}$ although a more detailed study in 1992 did find some minor abnormalities of ciliary function. ${ }^{115}$ It was proposed that Young's syndrome was caused by childhood mercury exposure (also responsible for Pink disease (acrodynia) which is a recognised aetiology of bronchiectasis for some UK patients) on the grounds that the specific type of obstructive azoospermia reported in Young's syndrome is becoming less common and epidemiologically this fits with the withdrawal of mercury-containing products such as teething powders in the UK and Australia. ${ }^{116}$ The role of Young's syndrome in the aetiology of bronchiectasis may perhaps become of historical interest only.

\section{Alpha-1 antitrypsin deficiency}

PiZZ A1AT deficiency has been found in $<1 \%$ of patients presenting with bronchiectasis. ${ }^{62}$ Insufficient information is given in aetiological studies to guide which bronchiectasis patients to investigate for A1AT deficiency.

\section{Congenital causes}

Congenital abnormalities causing bronchiectasis and presenting in adults are rare. Such congenital abnormalities may include Williams Campbell syndrome (bronchomalacia), Mounier Kuhn syndrome (tracheobronchomegaly) and lung sequestration.

The diagnosis of both Williams Campbell and Mounier Kuhn syndromes are made based on characteristic features on CT scanning of the chest. ${ }^{117}{ }^{118}$ The data describing the incidence of these syndromes presenting in adulthood is limited to case reports or series.

\section{Investigations of lung microbiology}

The main role of sputum culture is to help guide the use of antibiotics both for treatment of acute exacerbations and for chronic suppressive therapy. In addition the detection of some micro-organisms may also help in identifying an underlying aetiology. For example isolation of environmental mycobacteria should prompt further investigations for the presence of non-tuberculous mycobacteria (NTM) pulmonary disease; finding Staphylococcus aureus might indicate the presence of cystic fibrosis; isolation of A. fumigatus may prompt investigations for ABPA.

There has not been a systematic analysis of the role of bronchoscopy in the diagnosis and management of bronchiectasis. However, it should be considered in those patients who do not expectorate especially in the context of infective change seen on CT. It may be particularly helpful in the diagnosis of NTM pulmonary disease. See appendix 7 for studies of lower respiratory tract micro-organisms.

\section{Evidence statements}

It is sometimes possible to define an aetiological cause for bronchiectasis which directly alters management through the use of a standard panel of investigations. $(2++)$

Allergic bronchopulmonary aspergillosis (ABPA) occurs in 4\% (range 1\%-8\%) of patients with bronchiectasis. $(2+)$

A diagnosis of ABPA is supported by total IgE $>500 \mathrm{IU} / \mathrm{mL}$ $(\mathrm{ku} / \mathrm{L})$ and either positive Aspergillus-specific IgE or immediate reaction on skin prick testing. (4)

Antibody deficiency syndromes are found in 7\% (range $2 \%-17 \%)$ of patients with bronchiectasis. $(2+)$

Cystic fibrosis is diagnosed in 3\%-4\% of adult patients with bronchiectasis. $(1+)$

Primary ciliary dyskinesia is diagnosed in the majority of studies in $\leq 5 \%$ of adult patients with bronchiectasis. $(1+)$

There is little evidence to support routine testing for autoimmune disease or alpha one antitrypsin deficiency in the absence of suggestive clinical features. $(2++)$

Serum rheumatoid factor is frequently positive in patients with bronchiectasis $(2+)$ but the clinical significance of this is uncertain.

Aspiration is often associated with bronchiectasis $(2+)$ and may be causally related. (4) 


\section{Recommendations (see table 1)}

$>$ A panel of investigations should be performed to establish the underlying cause of bronchiectasis. (B)

a. Co-morbidities and past medical history should be recorded in patients diagnosed with bronchiectasis to identify relevant and possibly causative disease such as rheumatoid arthritis, COPD, asthma, gastro-oesophageal reflux disease and inflammatory bowel disease. (C)

b. Measure full blood count, serum total IgE and assessment of sensitisation (specific IgE or skin prick test) to $A$. fumigatus in all patients with bronchiectasis. (D)

c. Serum IgG, IgA and IgM should be performed in all patients with bronchiectasis. (C)

d. Consider measuring baseline specific antibody levels against capsular polysaccharides of $S$. pneumoniae in all patients to investigate for specific antibody deficiency. If pneumococcal antibodies are low, immunise with 23 valent polysaccharide pneumococcal vaccine, followed by measurement of specific antibody levels $4-8$ weeks later. (D).

e. Test for cystic fibrosis (according to NICE Guidelines for $\mathrm{CF}$ ) in patients with supporting clinical features, for example, early onset, male infertility, malabsorption, pancreatitis. (B)

f. Test for PCD (according to ERS Guidelines for PCD Diagnosis) in patients with supporting clinical features, including a history of neonatal distress, symptoms from childhood, recurrent otitis media, rhinosinusitis, or infertility. (A)

g. Sputum cultures should be performed in all patients with bronchiectasis for routine and mycobacterial culture. (D)

\section{Good practice points}

$\checkmark$ A previous diagnosis of idiopathic bronchiectasis should prompt careful reinvestigation for a primary cause in the context of a deteriorating clinical course or a young patient (usually considered to be age 50 and under but not limited to this age group).

$\checkmark$ Referral to a specialist centre for investigation should be considered for young patients (usually considered to be age 50 and under but not limited to this age group) and those with apparent idiopathic bronchiectasis especially where there is evidence of progressive disease.

$\checkmark$ Consider testing for RF, anti CCP, ANA and ANCA in patients with coexisting clinical features of arthritis, connective tissue disease and/or systemic vasculitis.

$\checkmark$ Consider testing for A1AT deficiency in patients with coexisting basal panacinar emphysema.

$\checkmark$ Investigations for reflux and aspiration should be undertaken only in symptomatic patients, or where there are other suggestive clinical features.

$\checkmark$ Consider bronchoscopy for patients with localised disease to rule out an endobronchial lesion or foreign body as the cause of bronchiectasis.

$\checkmark$ A bronchial aspiration or bronchial wash targeting the areas of bronchiectasis from CT scan of the chest should be considered in patients who do not expectorate and can be particularly helpful in the diagnosis of NTM pulmonary disease.

$\checkmark$ Serum protein electrophoresis should be performed in all patients with bronchiectasis with raised immunoglobulins.

$\checkmark$ Consider HIV-1 serology in patient with bronchiectasis depending on prevalence of HIV-1 and clinical features suggestive of increased risk of retroviral infection.

\section{Research recommendations}

Consensus criteria for diagnosis of ABPA need to be validated in bronchiectasis cohorts.

Consensus criteria for definition of abnormal post pneumococcal test immunisation antibody responses need to be validated in bronchiectasis cohorts.

\section{SECTION 7}

\section{Prognosis: what is the outlook for these patients?}

A UK epidemiology study examined mortality and noted the age adjusted mortality rate for women with bronchiectasis to be 1438 per 100000 against 636 for the general population (comparative mortality of 2.26) and, in men, the age adjusted mortality rate for bronchiectasis population was 1915 per 100000 compared with 895 for the general population (comparative mortality of 2.14). ${ }^{4}$

A comprehensive assessment by Martinez-Garcia et al ${ }^{119}$ 2014 in Spanish patients comparable to the UK population introduced the mortality prediction score (FACED), based on a study showing that of 154 deaths during follow-up, $42.9 \%$ were attributed to respiratory disease with $9.1 \%$ due to neoplasia and 9.1\% to cardiovascular disease. ${ }^{119}$ Similarly the Bronchiectasis Severity Index (BSI) tool was based on studies of outcome in patients with bronchiectasis across several different centres, and the study reported a mortality rate of $62(10.2 \%)$, directly relevant to bronchiectasis in 32 cases (listed as exacerbation of bronchiectasis, end-stage bronchiectasis, pneumonia, LRTI, chronic respiratory failure or pneumothorax). ${ }^{120}$ Otherwise, myocardial infarction was the cause of death in 12 cases, malignancy in eight cases, and other causes included heart failure, stroke, other sepsis, pulmonary embolism, trauma, alcoholic liver disease and post-operative complications.

\section{SECTION 8}

\section{Severity scoring}

The availability of the two clinical scoring systems, the Bronchiectasis Severity Index (BSI) (table 4) ) $^{120}$ and FACED ${ }^{119}$ (see tables 4 and 5), has increased our ability to predict future mortality in patients with bronchiectasis. The BSI additionally provides information on morbidity, hospital admissions and exacerbations. As bronchiectasis is not a rapidly fatal condition, these latter outcomes are regarded as more important for clinical decision making. Reassuringly, being derived in different cohorts, the two scores include similar variables. The major limitation of the FACED score is the absence of exacerbations which are regarded by many clinicians as the most important modifiable marker of severity. Prior exacerbations are also the strongest predictor of future exacerbations which explains why a number of studies have found FACED to be poorly predictive of future morbidity. ${ }^{121-123}$ For this reason the BSI is the preferred scoring system. At the time of writing, modifications of the FACED score to include exacerbations are undergoing evaluation. There may be some relevant omissions from both scores, notably the presence of co-morbidities which are important in the prognosis of other chronic respiratory diseases.

FACED includes $\mathrm{FEV}_{1}$, Age, $P$. aeruginosa colonisation, Extent of bronchiectasis (counting lobar involvement on CT) and Dyspnoea (using the modified MRC scale). All the variables are dichotomised and scored 0 vs 1 or 2 . The 5 year mortality in mild, moderate and severe disease (defined as a score of $0-2,3-4$ and $5-7$ points respectively) is $4 \%, 25 \%$ and $56 \%$ respectively. It has been shown to predict 5 year mortality, although the study used the score calculated at the time of initial diagnosis (and is not, therefore, designed for follow-up of existing patients, an 
Table 4 Variables involved in calculating the severity score in the Bronchiectasis severity index

\begin{tabular}{|c|c|c|c|c|}
\hline \multirow[b]{2}{*}{ Age (years) } & \multicolumn{4}{|c|}{ Factor and points for scoring system } \\
\hline & $<50$ (0 points) & 50-69 (2 points) & $\begin{array}{l}70-79 \\
\text { (4 points) }\end{array}$ & $>80$ (6 points) \\
\hline BMI $\left(\mathrm{Kg} / \mathrm{m}^{2}\right)$ & $<18.5$ (2 points) & $18.5-25$ (0 points) & $\begin{array}{l}26-30 \text { (0 } \\
\text { points) }\end{array}$ & $>30$ (0 points) \\
\hline $\mathrm{FEV}_{1} \%$ predicted & $>80$ (0 points) & $50-80$ (1 point) & $\begin{array}{l}30-49 \\
\text { (2 points) }\end{array}$ & $<30$ (3 points) \\
\hline Hospital admission within last 2 years & No (0 points) & & Yes (5 poir & \\
\hline Number of exacerbations in previous 12 months & 0 (0 points) & $1-2$ (0 points) & $\geq 3$ (2 poin & \\
\hline MRC breathlessness score & $1-3$ (0 points) & 4 (2 points) & 5 (3 point & \\
\hline P. aeruginosa colonisation & No (0 points) & & Yes (3 poir & \\
\hline Colonisation with other organisms & No (0 points) & & Yes (1 poir & \\
\hline Radiological severity & $<3$ lobes affected ( 0 points) & $\geq 3$ lobes or cystic $b$ & any lobe $(1$ & \\
\hline
\end{tabular}

0-4 Points=mild disease; 5-8=moderate disease; 9 and over=severe disease.

important limitation) and it is not known whether a change in score reflects a change in prognosis.

The BSI combines age, body mass index, $\mathrm{FEV}_{1}$, previous hospitalisation, exacerbation frequency, colonisation status and radiological appearances. The score was designed to predict future exacerbations and hospitalisations, healthstatus, and death over 4 years. Patients with NTM were excluded. Data were provided which examine how the score performed in annual prediction of events, a more relevant clinical scenario than provided for FACED, and the team have developed a useful online calculator (available at www.bron chiectasisseverity.com). A score of $0-4$ (mild) is associated with $0 \%-2.8 \%$ mortality and $0 \%-3.4 \%$ hospitalisation rate over 1 year, and $0 \%-5.3 \%$ mortality and $0 \%-9.2 \%$ hospitalisation rate over 4 years.

Outcomes in moderate bronchiectasis (score 5-8) were $0.8 \%-4.8 \%$ mortality and $1.0 \%-7.2 \%$ hospitalisation rate at 1 year and 4\%-11.3\% mortality and 9.9\%-19.4\% hospitalisation rate at 4 years. A score over 9 (severe bronchiectasis) was associated with 1 year 7.6\%-10.5\% mortality, and $16.7 \%-$ $52.6 \%$ hospitalisation rate and a 4 year $9.9 \%-29.2 \%$ mortality and $41.2 \%-80.4 \%$ hospitalisation rates. Understanding the factors associated with future morbidity and mortality in bronchiectasis provides a rationale, discussed further elsewhere, for which variables should be assessed and monitored in bronchiectasis.

\section{Good practice point}

$\checkmark$ Consider using the bronchiectasis severity index which may help guide management.

\section{SECTION 9}

\section{Stable state treatment}

Airway clearance techniques

Respiratory physiotherapy aims to mobilise secretions and aid effective expectoration, improving airway clearance and providing some control of cough. ${ }^{124}$ The other aims of respiratory physiotherapy include improving efficiency of ventilation, maintaining or improving exercise tolerance, improving knowledge and understanding, and reducing breathlessness and (thoracic) pain. ${ }^{125}$ The physiotherapist can discuss step up and step down airways clearance techniques in managing exacerbations. In addition, the respiratory physiotherapist can assist patients who require management of continence issues and musculoskeletal dysfunction. ${ }^{126}$ In this way, a physiotherapist aims to optimise a patient's physical function.

Which patients should be taught airway clearance techniques? A single randomised crossover trial has been published which assesses the efficacy of regular respiratory physiotherapy versus no respiratory physiotherapy in participants with bronchiectasis and chronic sputum expectoration. ${ }^{124}$ Twenty stable outpatients who did not practice regular respiratory physiotherapy were included. The intervention comprised of twice daily 20-30 min sessions of respiratory physiotherapy using an oscillating positive expiratory pressure device (Acapella Choice) over a 3 month period. There was a significant improvement in all domains and the total Leicester Cough Questionnaire (LCQ) score with regular respiratory physiotherapy (median (IQR) $(1.3(-0.17-$ 3.25 ) units; $\mathrm{P}=0.002)$. It should be noted that the MCID for LCQ is 1.3 units. ${ }^{127}$ Sputum volume measured over 24 hours increased significantly with regular respiratory physiotherapy (2 $(0-6) \mathrm{mL} ; \mathrm{P}=0.02)$ as did exercise capacity as measured by the incremental shuttle walk test (ISWT) $(40$ (15-80) $\mathrm{m} ; \mathrm{P}=0.001)$. There was also a significant improvement in quality of life (QoL) with a significant improvement in the St George's Respiratory Questionnaire (SGRQ) total score (7.77 (-0.99-14.5) unit improvement; $\mathrm{P}=0.004)$. No sham treatment was provided in the no respiratory physiotherapy arm and so there is some potential for a placebo effect. However, this study does provide low-quality evidence that regular twice daily respiratory physiotherapy is effective in stable patients.

\begin{tabular}{|c|c|c|}
\hline \multirow[b]{2}{*}{$\mathrm{FEV}_{1} \%$ predicted } & \multicolumn{2}{|c|}{ Factor and points for scoring system } \\
\hline & $<50$ (2 points) & $\geq 50$ (0 points) \\
\hline Age (years) & $\leq 70$ ( 0 points) & $>70$ (2 points) \\
\hline Colonisation by $P$. aeruginosa & No (0 points) & Yes (1 point) \\
\hline $\begin{array}{l}\text { Radiological extension of } \\
\text { bronchiectasis }\end{array}$ & $1-2$ lobes (0 points) & $>2$ lobes ( 1 point) \\
\hline Modified MRC dyspnoea scale & $1-2$ (0 points) & III-IV (1 point) \\
\hline
\end{tabular}

0-2 Points=mild disease; 3-4=moderate disease; 5-7=severe disease. 
A consecutive before-after case series assessed the effect of bronchopulmonary hygiene physical therapy (BHPT) on health-related QOL in 53 stable outpatients with bronchiectasis ${ }^{128}$ ). BHPT comprised two sessions at least 2 weeks apart and included education and instruction on an appropriate airway clearance technique to be used at home. The LCQ and cough symptom severity visual analogue score (VAS) were administered at initial assessment and more than 4 weeks later. At the follow-up visit, all patients reported administering their BHPT regime at least once per day. After BHPT, there were significant improvements in cough symptoms (mean cough VAS before 43.3 (3.6) vs. after 27.5 (3.1); mean difference 15.8; $95 \%$ CI of difference 9.6 to $22 ; \mathrm{P}<0.0001)$ and cough-related health status (mean LCQ total score before 14.2 vs. after 17.3; mean difference 3.1; $95 \%$ CI of difference 2.4 to 3.9 ; $\mathrm{P}<0.001)$. Limitations to the study including the lack of a control group and the use of only subjective outcome measures. In addition, such a variety of components were included as part of BHPT that the efficacy of individual components has not been determined.

\section{Adherence}

In an observational, non-comparative study, 75 patients with bronchiectasis and $P$. aeruginosa were reviewed over a year to determine if baseline beliefs about treatment, clinical factors and QoL predicted adherence. ${ }^{129} 41 \%$ of patients were adherent to airway clearance. Younger participants and those with a decreased understanding of the necessity of airway clearance were more likely to have reduced adherence.

\section{Evidence statement}

Regular twice daily respiratory physiotherapy increases sputum expectoration, improves cough-related health status, quality of life and exercise capacity in individuals with stable bronchiectasis and chronic sputum expectoration (1-)

Bronchopulmonary hygiene therapy (including education and instruction of an appropriate airway clearance technique) at least once per day does lead to an improvement in cough symptoms and cough-related health status in stable outpatients with bronchiectasis (3)

Younger patients (usually considered to be age 50 and under but not limited to this age group) and those with decreased understanding of the necessity of airway clearance may have reduced adherence levels (3)

\section{Recommendation}

$>$ Teach individuals with bronchiectasis to perform airway clearance. (D)

\section{Good practice points}

$\checkmark$ Airway clearance techniques should be taught by a respiratory physiotherapist.

$\checkmark$ At initial assessment, a respiratory physiotherapist should educate the patient about their condition and if appropriate give advice on adjuncts (inhaled/oral therapy or exercise) that may enhance effectiveness of their chosen airway clearance technique.

$\checkmark$ Patients admitted with an exacerbation of bronchiectasis should be seen daily by a respiratory physiotherapist until their airway clearance is optimised.

\section{Which airway clearance techniques should be taught?}

There are a number of airway clearance techniques that can be used in the management of bronchiectasis. However, it is beyond the scope of this guideline to describe each airway clearance technique and the reader is referred to detailed descriptions of the techniques for further information. ${ }^{130}$

In 2002, a survey of the current physiotherapy management of patients with bronchiectasis in the UK found that $91 \%$ of senior physiotherapists taught the active cycle of breathing techniques (ACBT) routinely. ${ }^{131}$ Other techniques such as positive expiratory pressure (PEP), oscillating positive expiratory pressure (OPEP), autogenic drainage $(\mathrm{AD})$ and intermittent positive pressure breathing (IPPB) were used much less frequently. ${ }^{131}$ Most respondents also used ambulation, exercise and education on the use of inhaled therapy. ${ }^{131}$

\section{Active cycle of breathing techniques}

The active cycle of breathing techniques (ACBT) is the most commonly taught airway clearance technique in patients with bronchiectasis. ${ }^{131}$ It is often used in conjunction with manual techniques (MTs), postural drainage (PD) or modified postural drainage (MPD). Many of the studies use a combination of ACBT and PD, making evaluation of ACBT alone difficult. A randomised crossover single intervention study by Syed et al compared ACBT to conventional chest physical therapy (CPT). ${ }^{132}$ Both groups completed $10-15$ min of postural drainage (PD) prior to their intervention. ACBT was completed with $\mathrm{PD}$ as per 'evaluation' (although no details were given on which positions this included) and CPT was completed in PD positions (again no details of these positions) with percussion and shakes completed by the physiotherapist. There were no significant differences in outcomes between the techniques in terms of sputum weight, sputum volume or FEV 1 and FVC. Patients however, did find ACBT more comfortable as measured on a visual analogue scale. A number of studies have also shown that when measuring sputum weight, ACBT is as effective as oscillating PEP. ${ }^{133-135}$ In contrast, a study comparing ACBT to the test of incremental respiratory endurance (TIRE) device found that ACBT (plus PD and vibration) was more effective than the TIRE. ${ }^{136}$

One study looked at the use of ACBT in an exacerbation. ${ }^{137}$ Thirty patients with an exacerbation of bronchiectasis were randomly assigned to either ACBT or conventional respiratory physiotherapy (CCPT- diaphragmatic breathing in PD positions with manual techniques). All patients received 14 days of intravenous antibiotics and evaluations were completed at the beginning and end of the admission. The ACBT group had significantly improved measures of the impact of cough on quality of life (Leicester Cough Questionnaire) in both the physical domain of the LCQ $(6 \mathrm{v} 4(\mathrm{P}=0.023))$ and the total LCQ score $(14 \mathrm{v} 12$ $(\mathrm{P}=0.019))$ when compared with CCPT). There were no statistically significant differences between groups in other measures including spirometry and modified MRC dyspnoea scores.

\section{Manual techniques}

Manual techniques are among the longest-standing airway clearance techniques and include percussion, shaking, vibrations and over pressure. These techniques are used clinically to both loosen secretions as well as to reduce fatigue or increase effectiveness of other airway clearance techniques. ${ }^{138}$

A single intervention study $(n=22)$ over 4 days ${ }^{139}$ looked at the sputum cleared from three different techniques; (1) postural drainage (PD) and coughing (2) PD, percussion and coughing and (3) PD and 'huffing'. These three techniques were compared 
with cough technique alone. Techniques were randomly allocated and completed in three $20 \mathrm{~min}$ sessions (separated by $10 \mathrm{~min}$ rest periods) on the same day. Sputum was collected at baseline, and after each $20 \mathrm{~min}$ session at $30 \mathrm{~min}$ (session one), $60 \mathrm{~min}$ (session two) and $90 \mathrm{~min}$ (session three). PD, percussion and coughing cleared more sputum at $60 \mathrm{~min}$ (session two) and $90 \mathrm{~min}$ (session three) compared with the other techniques. Additionally, PD, percussion and coughing and PD and huffing cleared the most secretions with the worst rheological properties. However, this clearance of the more viscous sputum only occurred when these techniques were performed for two 20 min sessions in succession, indicating a cumulative effect.

\section{Postural drainage}

Postural drainage (PD) is the use of gravity-assisted positioning to drain areas of the lung. PD specifically uses gravity to drain the lower and middle lobes using a head down tilt. ${ }^{130}$

A single treatment of ACBT plus PD was compared with ACBT performed in the sitting position and with the Flutter in the sitting position. The treatment with PD yielded a sputum wet weight twice that of either ACBT in sitting or the Flutter in sitting. Subjects rated their preference for techniques as $44 \%$ for the Flutter, 22\% for the ACBT in sitting and 33\% for the ACBT plus PD. Treatment with PD was associated with significantly more discomfort than the treatments in sitting and was felt to interfere more with daily life than the Flutter and more time consuming.

Many clinicians have concerns about the impact of head-down tilt PD positions on symptoms of gastro-oesophageal reflux disease (GORD). There are no studies assessing the impact of $\mathrm{PD}$ on GORD in the bronchiectasis population.

\section{Modified postural drainage}

There is no evidence as to whether using $\mathrm{mPD}$ does reduce symptoms of GORD or breathlessness in bronchiectasis. There is also no evidence to assess the impact of mPD compared with PD (head down tilt) on the effectiveness of airway clearance.

\section{Positive expiratory pressure}

There are no studies formally assessing the effect of positive expiratory pressure (PEP) as an ACT in bronchiectasis. A study evaluated the effect of a single session of PEP therapy in the sitting position on gastro-oesophageal reflux. ${ }^{140} \mathrm{~A}$ session of PEP therapy in sitting was not associated with a significant increase in reflux by $\mathrm{pH}$ monitoring.

\section{Oscillating PEP}

In bronchiectasis, the oscillating PEP (OPEP) devices that have most commonly appeared in the literature to date are the Flutter and Acapella devices, which appear to have similar performance characteristics. ${ }^{141}$

In a 4 week crossover trial, the Flutter (combined with the Forced Expiratory Technique (FET) and PD as necessary) was shown to be as effective as the ACBT and PD for median weekly sputum weight when used twice daily. ${ }^{133}$ Neither of the techniques (ACBT or Flutter) had an adverse effect on PEF or breathlessness. Eleven of the 17 subjects expressed a preference for the Flutter.

In a previously described single intervention randomised crossover study comparing Flutter, ACBT and ACBT with PD there were no differences in effectiveness as measured by sputum weight and $\mathrm{FEV}_{1}$ between ACBT and Flutter groups. ${ }^{135}$ However, although ACBT and PD was the most effective technique in terms of sputum weight, the Flutter was the most preferred by patients (44\% preferred Flutter, 22\% ACBT and 33\% ACBT plus PD).

In a pilot study in Hong Kong, 15 patients with an acute exacerbation of bronchiectasis were randomly allocated to three groups: the Flutter plus deep breathing and coughing $(n=5)$, deep breathing and coughing plus postural drainage $(n=5)$ and deep breathing and coughing alone $(n=5) .{ }^{142}$ There were no differences among the three groups in sputum production or lung function parameters. Patients reported that all techniques were equally easy to use, but again the Flutter was perceived as being the most effective.

A study in 15 stable bronchiectasis patients, looked at outcomes before and after 3 weeks of daily OPEP (Aerobika) use. This showed significant improvements in Patient Evaluation Questionnaire (PEQ)-ease bringing up sputum $(P=0.048)$ and PEQ-global assessment $(\mathrm{P}=0.01)$. Ventilation defect percent (VDP) was also evaluated by hyperpolarised MRI, however no significant changes were observed. ${ }^{143}$

A systematic review evaluated OPEP devices in bronchiectasis. ${ }^{144}$ In the seven studies reviewed ( $n=146$ patients), OPEP therapy was associated with improvements in sputum expectoration and quality of life measures compared with no treatment. Moreover they concluded that compared with other ACTs, the effects in terms of sputum expectoration, lung function, gas exchange, and symptoms were equivalent. However, the authors did suggest a greater patient preference for oscillating PEP compared with ACBT with or without Gravity Assisted Positioning (GAP).

\section{Autogenic drainage}

A randomised crossover study $(n=31)$ in stable bronchiectasis, compared autogenic drainage (AD) to slow expiration with glottis opened in lateral posture (ELTGOL) and temporary positive expiratory pressure (TPEP). ${ }^{145}$ During physiotherapy sessions, $\mathrm{AD}$ and ELTGOL expectorated more sputum than TPEP (AD v TPEP, $3.1 \mathrm{~g}$ (95\% CI 1.5 to 4.8$)$ ). $48.8 \%$ of participants stated a preference for AD. The LCQ improved with all techniques, although this did not reach the mean clinically important difference (MCID) for any of the techniques.

\section{Inspiratory muscle training}

The test of incremental respiratory endurance is primarily used for inspiratory muscle training. However, it has been proposed as a method of airway clearance in bronchiectasis. ${ }^{136}$

A randomised crossover pilot study $(n=30)$ compared the effects of a single session of the Acapella (in sitting with huffing included) to a single session of a threshold inspiratory muscle trainer (IMT) (in sitting with huffing included). ${ }^{146} \mathrm{~A}$ statistically significant difference was found in sputum volume expectorated with the Acapella treatment $(7.16 \pm 1.12 \mathrm{~mL})$ compared with IMT $(6.46 \pm 1.08 \mathrm{~mL})$ with a mean difference of $0.7 \mathrm{~mL}(\mathrm{P}=0.014)$. Although statistically significant, a difference of $0.7 \mathrm{~mL}$ is unlikely to be of any clinical significance. In addition, using a patient preference scale, patients felt that the Acapella was more useful in terms of clearing secretions $(\mathrm{P}<0.05)$.

\section{High frequency chest wall oscillation}

High frequency chest wall oscillation (HFCWO) is the application of positive pressure air pulses to the chest wall using an inflatable vest. A single randomised controlled trial assessed the effectiveness of HFCWO over a 15 day period. Thirty subjects were randomised into three groups; HFCWO, traditional 
techniques of respiratory physiotherapy (CPT) (including PEP mask, bottle PEP and oscillating PEP) and a control group (medical therapy only). HFCWO improved breathlessness, quality of life, sputum volume (from $62.5 \pm 18.9 \mathrm{~mL}$ at admission to $70.0 \pm 21.1$ in the conventional physiotherapy group and from $52.0 \pm 16.9 \mathrm{~mL}$ to $72.5 \pm 24.0 \mathrm{~mL}$ in the HFCWO group). and lung function significantly more than traditional respiratory physiotherapy.

\section{Intrapulmonary percussive ventilation}

Intrapulmonary percussive ventilation (IPV) is the delivery of a pulsatile flow of gas to the lungs during inspiration which vibrates the airway walls. A single randomised crossover study $(n=22)$ compared the effectiveness of a single session of IPV in sitting with a single session of chest physical therapy (CPT) in stable bronchiectasis. ${ }^{147}$ There were no statistically significant differences between groups in the amount of sputum expectorated or dyspnoea score after treatment. There were no adverse events with either treatment that were severe enough to require discontinuation. IPV was associated with less discomfort than CPT.

\section{Intermittent positive pressure breathing}

Intermittent positive pressure breathing (IPPB) provides positive pressure throughout inspiration, thereby augmenting tidal volume. ${ }^{148}$ There is very little evidence investigating the effects of IPPB in bronchiectasis. A single randomised crossover study $(n=18)$ compared the use of IPPB and negative pressure ventilation (NPV) given once per week over a 4 week period. ${ }^{149}$ Pulmonary function and walking distance did not change significantly after either treatment.

\section{Exercise}

A study investigated the impact of an 8 week pulmonary rehabilitation programme. ${ }^{150}$ The authors suggested a small but significant impact on exacerbation frequency over 12 months in the intervention group (PR) (median 1 (IQR 1-3)) compared with physiotherapy alone (median 2 (IQR 1-3)) $(\mathrm{P}=0.012)$.

\section{Evidence statements for airway clearance techniques}

The active cycle of breathing techniques is as effective as oscillating positive expiratory pressure (Flutter and Acapella) at clearing sputum. (1-)

The active cycle of breathing techniques plus postural drainage enhances the quantity of sputum expectorated compared with the active cycle of breathing techniques in the sitting position or oscillating positive expiratory pressure (Flutter) in the sitting position. (1-)

The active cycle of breathing techniques (plus postural drainage and vibration) is more effective at clearing sputum than the test of incremental respiratory endurance. (1-)

Oscillating positive expiratory pressure (Acapella) is more effective at clearing sputum than a threshold inspiratory muscle trainer. (1-)

Oscillating positive expiratory pressure (Acapella) improves QoL, sputum volume expectorated and exercise capacity compared with no airway clearance technique over a 3 month period. (1-)

High frequency chest wall oscillation improves breathlessness, quality of life, sputum volume and lung function. (1-)

\section{Recommendations}

$>$ Offer active cycle of breathing techniques or oscillating positive expiratory pressure to individuals with bronchiectasis. (D)

> Consider gravity assisted positioning (where not contraindicated) to enhance the effectiveness of an airway clearance technique. (D)

\section{Good practice points}

$\checkmark$ CT imaging should be reviewed to complement the physiotherapy assessment. Where indicated, this information could be used in order to teach the patient the appropriate postural drainage position(s) for their affected bronchopulmonary segment(s).

$\checkmark$ Patients should be made aware of the range of available airway clearance techniques.

$\checkmark$ Consider patient preference and adherence when recommending an airway clearance technique.

$\checkmark$ Consider the inclusion of the forced expiration technique (huff) should be considered for all airway clearance techniques.

$\checkmark$ Consider modified postural drainage (no head down tilt) in patients for whom postural drainage is contraindicated or not tolerated.

$\checkmark$ If symptoms of gastro-oesophageal reflux increase with modified postural drainage (no head down tilt), an airway clearance technique in the sitting position should be taught.

$\checkmark$ Consider autogenic drainage, positive expiratory pressure, high frequency chest wall oscillation and intrapulmonary percussive ventilation as an alternative airway clearance technique if other techniques are not effective or acceptable to the patient.

$\checkmark$ Patients should be encouraged to perform regular physical exercise (plus the forced expiration technique/huff) to promote airway clearance.

$\checkmark$ If there is ongoing haemoptysis, refer back to the respiratory physiotherapist to determine the optimum airways clearance technique.

\section{Evidence statement}

Oscillating positive expiratory pressure (Acapella) (plus postural drainage) is effective and safe to use during an acute exacerbation. (1-)

\section{Good practice points}

$\checkmark$ Manual techniques may be offered to enhance sputum clearance when the patient is fatigued or undergoing an exacerbation.

$\checkmark$ Consider intermittent positive pressure breathing or non-invasive ventilation during an acute exacerbation to offload the work of breathing so fatigued and/or breathless patients can tolerate a longer treatment session and can adopt postural drainage positions.

\section{Research recommendations}

Randomised controlled trials using clinically important outcome measures are required to assess the effectiveness of airway clearance techniques in varying severities of bronchiectasis.

Randomised controlled trials are required to evaluate the effects of airway clearance techniques in patients who are undergoing an exacerbation. 


\section{How often should patients carry out airway clearance techniques? How long should an airway clearance session last?}

\section{Duration}

Most clinicians would advocate the use of an airway clearance technique for a period specific to the individual. Commonly, not more than $30 \mathrm{~min}$ is recommended. The aim is to perform the airway clearance technique until two clear huffs or coughs are completed. However, this is not always practical in those who are extremely productive. It is important that a balance is found between making sure that the treatment is long enough to maximise airway clearance, but not so long that the patient becomes fatigued.

\section{Frequency}

The frequency of the airway clearance technique should be specific to the needs of the individual patient and probably increased during an infective exacerbation. There is no evidence to support a particular frequency with recommendations of once or twice daily treatment commonly given.

\section{Good practice points}

$\checkmark$ The frequency and duration of the airway clearance technique should be tailored to the individual and may alter during periods of exacerbation.

$\checkmark$ Advise individuals to perform their airway clearance technique for a minimum of 10 minutes (up to a maximum of 30 minutes). After this time they should continue until two clear huffs or coughs are completed, or until the patient is starting to become fatigued.

\section{How soon should the patient be reviewed after the initial assessment?}

Initial assessment may take up to an hour, with education and instruction of an appropriate airway clearance technique included. A review of the individual's ability to effectively carry out this technique should be undertaken within 3 months of this initial appointment. $^{126}$

\section{Good practice points}

$\checkmark$ Individuals that have been assessed and taught an airway clearance technique should be reviewed by a respiratory physiotherapist within 3 months of their initial assessment.

$\checkmark$ Individuals with bronchiectasis who are followed up in secondary care should be assessed by a respiratory physiotherapist as part of their annual clinical review to ensure their airway clearance regimen is optimised.

$\checkmark$ All individuals with a deterioration in their condition (increased frequency of exacerbations and/or worsening of symptoms) should have their airway clearance technique reviewed by a respiratory physiotherapist (See figure 1 management of the deteriorating patient).

\section{Mucoactives in bronchiectasis}

Mucoactive medications describe medications which may have a direct impact on the clearance of mucus from airways. These can be defined by their different methods of action including expectorants (aid and/or induce cough), mucolytics (thin mucus), mucokinetics (facilitate cough transportability), and mucoregulators (suppress mechanisms underlying chronic mucus hyper secretion, such as glucocorticosteroids). Bronchiectasis research has mainly focused on mucolytics (DNase), ${ }^{151} 152$ mucokinetics/ expectorants (humidification, normal/isotonic saline $(0.9 \%$ saline) (IS), hypertonic saline (3\% saline and above) (HS) ${ }^{153-158}$ and mannitol ${ }^{159} 160$ and there is a small amount of evidence in mucoregulators (erdosteine) ${ }^{161}$ and bromhexine. ${ }^{162}$

\section{Humidification}

A small study $(n=7)$ showed that 30 min of cold water, jet nebulising humidification via a facemask before respiratory physiotherapy (postural drainage and the forced expiration technique) significantly increased sputum yield and radio aerosol clearance compared with respiratory physiotherapy alone. ${ }^{154}$ A further study $(n=10)$ using warm air humidification for 3 hours a day also showed a significant increase in radiolabelled clearance in the treatment group compared with the control group. ${ }^{163}$

\section{Saline}

A small single study $(n=8)$ found that the use of nebulised IS immediately before respiratory physiotherapy (postural drainage and the forced expiration technique) yielded significantly more sputum than physiotherapy alone. ${ }^{153}$

A four way single intervention crossover study looking at ACBT alone, ACBT and terbutaline, ACBT and isotonic saline (IS) and ACBT and hypertonic saline (HS), suggested that HS gave an additional improvement to $\mathrm{QoL}, \mathrm{FEV}_{1}$, sputum weight, ease of expectoration and viscosity in addition to the benefit shown by IS over that of terbutaline or ACBT alone. ${ }^{158}$

A 4 week crossover study in 32 patients with bronchiectasis compared $7 \%$ HS to a placebo (IS). $\mathrm{FEV}_{1}$ improved in the HS group compared with the IS group by $15.1(1.8) \%(\mathrm{P}<0.01)$; FVC by $11.2(0.7) \%(\mathrm{P}<0.01)$, QoL as measured by SGRQ showed an improvement of 6 units $(\mathrm{P}<0.05)$ in the HS group. ${ }^{155}$ Moreover sputum viscosity (subjective pourability), ease of clearance (as measured by a visual analogue scale) and healthcare utilisation also all improved in the HS group compared with IS.

A long term study (12 months) of 40 participants was designed to investigate the impact of HS compared with IS when used with ACBT, demonstrated that both IS and HS saline had a positive impact on all domains of the SGRQ and the LCQ after three, six and 12 months, and on $\mathrm{FEV}_{1}$, with an improvement in the IS group of (mean) $90 \mathrm{~mL}$ (11 to 169$) ; \mathrm{P}=0.04$ compared with baseline. 157

In summary this evidence suggests that HS may improve QoL outcomes and sputum clearance in individuals with bronchiectasis, however it is unclear if this benefit is over and above that of IS.

\section{Mannitol}

Many studies have suggested an in vitro benefit of mannitol ${ }^{164}$ and have shown it to be tolerated in inhaled devices by individuals with bronchiectasis in feasibility studies. ${ }^{165}$ In a phase three study of 343 subjects with bronchiectasis, ${ }^{159}$ subjects were randomised into either placebo ( $40 \mathrm{mg}$ mannitol) versus treatment dose mannitol $(340 \mathrm{mg}$ ) over a 12 week intervention period. An improvement was seen in SGRQ in the mannitol group, however this was not a clinically significant change. A subgroup of participants $(n=123)$ who continued to a further 52 weeks open label study continuing on mannitol had a reduction in sputum plugging on CT scan. In a large phase 3 RCT in 461 patients with bronchiectasis over 12 months, ${ }^{160}$ randomised patients received either placebo (low dose mannitol) or mannitol $(400 \mathrm{mg}$ ) over 
12 months. No differences were seen in the primary end point of exacerbation frequency between the two groups at these time points. However, this study did show a prolongation in time to first exacerbation and a small improvement in quality of life in the mannitol group.

In view of the potential for bronchoconstriction, a challenge test with hyperosmolar agents is suggested. All included studies performed a challenge test to assess for bronchoconstriction and/or bronchial hyper-reactivity prior to use of the device/ medication. ${ }^{155-160}$ Those individuals who reported chest tightness, wheeze, and difficulty in breathing or had a $10 \%$ or $15 \%$ reduction in spirometry were withdrawn from the studies. All the studies suggested that such events were minimal thereby showing good tolerance of hypertonic saline and mannitol. The studies varied on the use of a pre dose bronchodilator. However clinical practice suggests that pre-treatment with a bronchodilator may be necessary for those with the potential for bronchial hyper-reactivity (eg, a diagnosis of asthma, or defined bronchodilator reversibility).

\section{Mucolytics}

Recombinant human DNase (DNase, dornase alpha, Pulmozyme) breaks down the DNA released at the site of infection by the neutrophils. DNA causes the sputum to become thick and tenacious and therefore inhaled DNase makes the sputum less viscid and therefore easier to expectorate. A multi-centre study in 349 patients with idiopathic bronchiectasis randomised patients to use either $2.5 \mathrm{mg}$ DNase versus a placebo. ${ }^{152}$ There was a significant increase in the rate of both protocol-defined exacerbations $(0.66$ rate of exacerbations per patient per 166 days compared with 0.56 rate for those in placebo group, relative risk $=1.17$ ) as well as an increase in non-protocol defined exacerbations (relative risk 2.01) within the DNase group. DNase had a small but negative effect on $\mathrm{FEV}_{1}(-3.6 \%$ in DNase v $1.7 \%$ in control, $\mathrm{P}=0.05$ )

There have been very few trials reviewing the use of oral mucolytics in bronchiectasis. One study $(n=88)$ reviewed the addition of bromhexine hydrochloride to an antibiotic during an acute infective exacerbation compared with a placebo. ${ }^{162}$ It is thought this medication may influence sputum clearance by increasing the production of serous mucus thereby making the sputum thinner and less viscous. Results suggest that this medication was effective, as the percentage change in sputum production was greater in the bromhexine group at 7,10 and 16 days (mean difference (MD) $-21.5 \mathrm{~mL}, 95 \% \mathrm{CI}-38.9$ to -4.1 at day 16). Moreover the difficulty in expectoration was also improved in the bromhexine group at day 10 (MD -0.53 , $95 \% \mathrm{CI}-0.81$ to -0.25 ) however it had no impact on $\mathrm{FEV}_{1}$. Bromhexine hydrochloride is not widely available in the UK and is not listed in the BNF.

Erdosteine is another mucolytic which is thought to modulate mucus production through the scavenging activity of free radicals. A study in 2007 compared the use of erdosteine and respiratory physiotherapy to respiratory physiotherapy alone over a 15 day period in a bronchiectasis population $(n=30) .{ }^{161}$ This study was small and of poor methodological quality due to limited control of bias. Small changes were seen in some subjective sputum characteristics and $\mathrm{FEV}_{1}$ in the erdosteine group.

In the UK from National Audits 2010 and 2011, Carbocysteine was commonly prescribed $(27 \%-30 \%) .{ }^{166}$ There are no randomised controlled trials to demonstrate benefit.

\section{Evidence statements}

Recombinant human DNase increases exacerbation frequency in bronchiectasis. $(1+)$

Isotonic and hypertonic saline can improve cough related quality of life QoL and health related quality of life (HQoL) in patients with bronchiectasis in addition to airway clearance. (1-)

Mannitol did not improve exacerbation frequency but was shown to increase the time to first exacerbation, demonstrated a small improvement in QoL and may reduce sputum plugging. $(1+)$

The use of humidification prior to airway clearance improves sputum yield and radiolabeled clearance, when used in short term interventions ( $>1$ month). (3)

Oral mucolytics can improve sputum expectoration. (1-)

\section{Recommendations}

> Do not routinely use recombinant human DNase in adults with bronchiectasis. (A)

$>$ Consider the use of humidification with sterile water or normal saline to facilitate airway clearance. (D)

\section{Good practice points}

$\checkmark$ Consider a trial of mucoactive treatment in patients with bronchiectasis who have difficulty in sputum expectoration.

$\checkmark$ Perform an airway reactivity challenge test when inhaled mucoactive treatment is first administered.

$\checkmark$ Consider pre-treatment with a bronchodilator prior to inhaled or nebulised mucoactive treatments especially in individuals where bronchoconstriction is likely (patients with asthma or bronchial hyper-reactivity and those with severe airflow obstruction $\mathrm{FEV}_{1}<1$ litre).

$\checkmark$ If carbocysteine is prescribed, a 6 month trial should be given and continued if there is ongoing clinical benefit.

See figures 3 and 4.

See appendix 2: Challenge test details

\section{Research recommendation}

Randomised controlled trials are needed to assess the long term impact of muco-active therapies.

\section{What is the evidence for long term anti-inflammatory therapies in bronchiectasis?}

Airway inflammation is dominated by neutrophils although there is evidence to implicate multiple other inflammatory cells and pathways in the pathophysiology of bronchiectasis. ${ }^{167} 168$

\section{Inhaled corticosteroids (ICS)}

In bronchiectasis, a systematic review conducted in 2009 identified six single centre randomised controlled trials. ${ }^{169}$ Two studies were crossover studies ${ }^{170}{ }^{171}$ while the other four were parallel group studies. ${ }^{172-175}$ All were double blind placebo controlled studies with the exception of Martinez et al 2006 which was open label. ${ }^{172}$ Studies used multiple difference steroid preparations and doses (beclomethasone $800 \mu \mathrm{g} / \mathrm{day}, 1500 \mu \mathrm{g}$, fluticasone $500 \mu \mathrm{g} /$ day and $1000 \mu \mathrm{g} /$ day). Study duration ranged from 4 to 6 weeks to 6 months. Two studies reported reductions in sputum volume with ICS treatment. ${ }^{170} 172 \mathrm{~A}$ small improvement in lung function was suggested by the meta-analysis $(90 \mathrm{~mL}$ for $\mathrm{FEV}_{1}$ and FVC) in studies of $\leq 6$ months but this was not statistically significant if the study that was not placebo controlled was removed. ${ }^{169}$ No reduction in exacerbations was identified. 


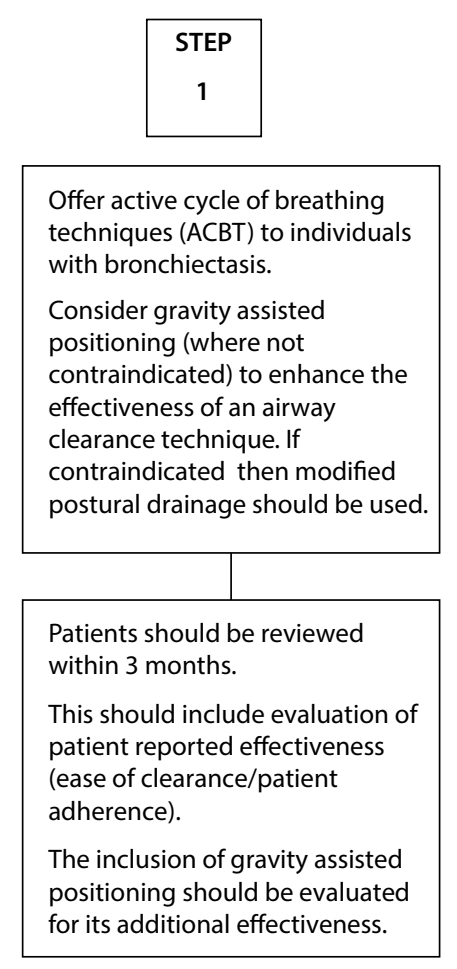

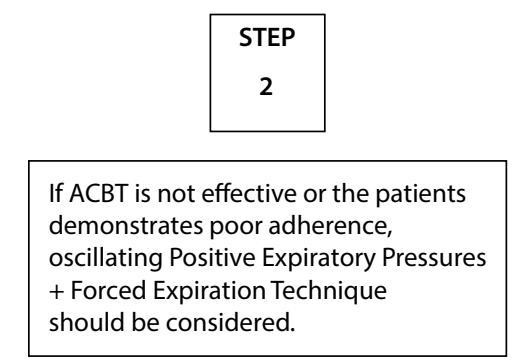

Figure 3 Physiotherapy management-stepwise airway clearance.

In longer term studies ( $>6$ months) no significant improvement in lung function or exacerbations were identified. ${ }^{169}$ The BTS guideline development group identified no new randomised controlled trials since 2009.

Clinically important adverse events associated with inhaled corticosteroids have been identified. Holme et al identified a high frequency of adrenal suppression associated with ICS treatment in bronchiectasis. ${ }^{176} \mathrm{~A}$ statistically significant increase in adverse effects was reported for high dose ICS in the trial of Martinez et al $(\mathrm{P}=0.04) .{ }^{172} \mathrm{~A}$ trial of high dose budesonide vs budesonide with formoterol identified a numerical increase in the frequency of local side effects with high dose ICS (pharyngeal irritation, dysphonia and gingivitis) and a single case of pneumonia which is a recognised adverse effect of inhaled corticosteroid treatment in COPD. ${ }^{177}$

\section{Oral corticosteroids}

No randomised controlled trials of oral corticosteroids either when stable or during exacerbations could be identified. ${ }^{178}$ No observational data were identified.

Increase airway clearance frequency. E.g.: from twice daily to three/four times daily.
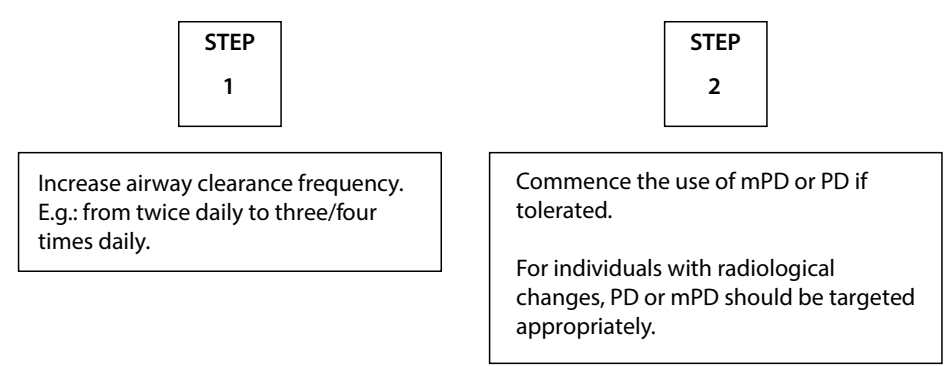

$\mathrm{PD}=$ postural drainage $\mathrm{mPD}=$ modified postural drainage

Figure 4 Airway clearance - exacerbations.
Phosphodiesterase four inhibitors (PDE4 inhibitors)

There are no randomised trials of PDE4 inhibitors in bronchiectasis. In addition, no observational data were identified.

\section{Methylxanthines}

No randomised controlled trials were identified in bronchiectasis for these drugs. ${ }^{179}$ No observational data were identified.

\section{Indomethacin}

A single trial of 25 adults with chronic lung diseases that included patients with bronchiectasis was identified. This study showed a significant reduction in sputum production over 14 days in patients that received inhaled indomethacin compared with placebo (difference $-75 \mathrm{~g}$ /day $95 \% \mathrm{CI}-134.6$ to -15.4 ). ${ }^{180}$ There were also significant improvements in dyspnoea. No further large trials have been performed.
If airway clearance is not effective then nebulised Isotonic ( $0.9 \%$ saline) or should be evaluated for its effectiveness pre-airway clearance (especially in patients with viscous secretions or

Individuals should be advised to complete Airway Clearance in the

- Bronchodilator

- Mucoactive treatment

- Nebulised antibiotic and/or inhaled steroids (if applicable)

ACBT: Active cycle of breathing techniques

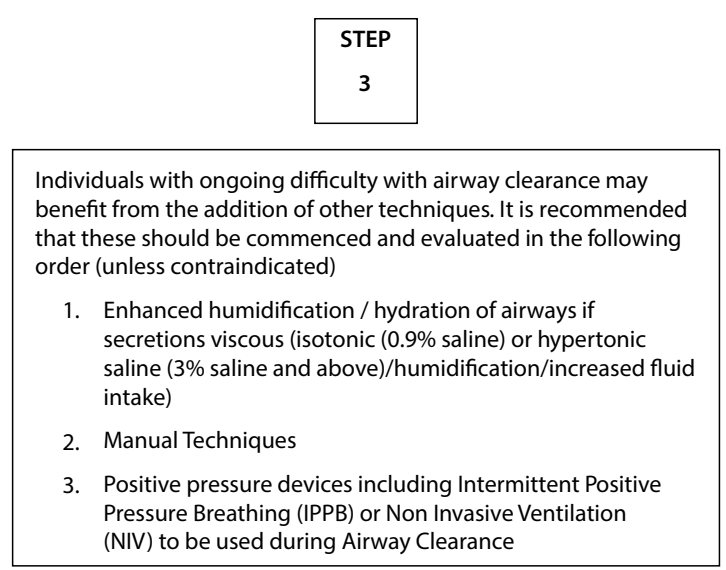


Leukotriene receptor antagonists

No randomised trials or observational studies of leukotriene receptor antagonists in bronchiectasis have been performed. ${ }^{181}$

\section{Neutrophil elastase inhibitors}

Neutrophil elastase is a proteolytic enzyme released by neutrophils which may have a role in disease progression. ${ }^{182}$ A single randomised controlled trial of 38 patients treated with a neutrophil elastase inhibitor for 4 weeks compared with placebo $(n=16)$ was identified. The study found no difference in sputum neutrophils but an improvement of $100 \mathrm{~mL}$ in the $\mathrm{FEV}_{1}$ in the NE inhibitor group $(\mathrm{P}=0.006) .{ }^{183}$ No larger trials have yet been performed.

\section{CXCR2 inhibitors}

A double blind randomised placebo-controlled trial of the CXCR2 antagonist AZD5069 in bronchiectasis for 28 days recruited 52 patients with a primary outcome of sputum neutrophil counts. This study showed a significant reduction in absolute neutrophil counts (69\% vs placebo, $\mathrm{P}=0.004)$. There was no significant difference in exacerbations or other clinical outcomes. Some sputum inflammatory markers e.g IL-6, GRO- $\alpha$, IL-1 $\beta$ and IL-8 increased with AZD5069 vs placebo. Four patients discontinued therapy in the active group vs 0 in the placebo group. ${ }^{184}$

\section{Statins}

Statins have multiple anti-inflammatory and immunodulatory effects in addition to their effects on cholesterol. A randomised placebo controlled trial has been performed in bronchiectasis. 60 patients were treated with atorvastatin $80 \mathrm{mg}$ daily for 6 months $(n=30)$ vs. placebo $(n=30) .{ }^{185}$ The primary endpoint was improvement in cough assessed by the Leicester Cough Questionnaire. The change from baseline to 6 months in LCQ score differed between groups, with a mean change of 1.5 units in patients allocated atorvastatin versus -0.7 units in those assigned placebo (mean difference $2 \cdot 2$, 95\% CI $0 \cdot 5-3 \cdot 9$; $\mathrm{P}=0 \cdot 01) .12(40 \%)$ of 30 patients in the atorvastatin group improved by 1.3 units or more on the LCQ compared with five $(17 \%)$ of 30 in the placebo group (difference $23 \%, 95 \% \mathrm{CI}$ $1-45 ; \mathrm{P}=0 \cdot 04)$. Ten $(33 \%)$ patients assigned atorvastatin had an adverse event versus three (10\%) allocated placebo (difference $23 \%$, 95\% CI 3-43; $\mathrm{P}=0 \cdot 02){ }^{185}$ No larger trials have been performed.

A randomised placebo cross-over trial investigated atorvastatin $80 \mathrm{mg}$ daily for 3 months in patients chronically infected with $P$. aeruginosa. Statin therapy did not improve the primary endpoint for improved cough assessed by the Leicester Cough Questionnaire. There were however significant improvements in the St. George's Respiratory Questionnaire (5.6 Unit improvement) and a reduction in serum CXCL8, TNF $\alpha$ and ICAM-1 in the statin treated group. ${ }^{186}$

\section{Evidence statements}

Inhaled corticosteroids may reduce sputum volume in bronchiectasis but are associated with adverse events including local and systemic effects. $(1+)$

There was insufficient evidence to evaluate the role of oral corticosteroids, PDE4 inhibitors, methylxanthines or leukotriene receptor antagonists in bronchiectasis. (4)

A small trial of inhaled indomethacin which included some patients with bronchiectasis showed reduced sputum volume and improved dyspnoea. (1-)
A small randomised trial of oral neutrophil elastase inhibitors in bronchiectasis showed improved FEV but no other benefits. (1-)

A small trial of CXCR2 antagonists showed reduced sputum neutrophil counts but no clinical benefits. (1-)

A small randomised trial of statins in bronchiectasis showed improved cough, but an increase in adverse events. (1-)

A small randomised trial of statins in bronchiectasis chronically infected with $P$. aeruginosa showed improved health related quality of life. (1-)

\section{Recommendations}

$>$ Do not routinely offer inhaled corticosteroids to patients with bronchiectasis without other indications (such as ABPA, chronic asthma, COPD and inflammatory bowel disease). (B)

> Do not offer long-term oral corticosteroids for patients with bronchiectasis without other indications (such as ABPA, chronic asthma, COPD, inflammatory bowel disease). (D)

> Do not routinely offer PDE4 inhibitors, methylxanthines or leukotriene receptor antagonists for bronchiectasis treatment. (D)

$>$ Do not routinely offer CXCR2 antagonists, neutrophil elastase inhibitors or statins for bronchiectasis treatment. (B)

\section{Good practice point}

$\checkmark$ Inhaled corticosteroids have an established role in the management of asthma and a proportion of patients with COPD which are common co-morbid conditions in bronchiectasis.

\section{Research recommendation}

Randomised controlled trials are needed to assess the long term impact of anti-inflammatory therapies.

\section{What treatments improve outcomes for patients with stable bronchiectasis?}

Long term antibiotic treatment ( $>=3$ months)

\section{Oral antibiotics}

Several randomised controlled studies have been performed to ascertain the effect of long term antibiotics in bronchiectasis. Some of the earliest were performed in the 1950s and so the diagnostic techniques defining the patient population (bronchiectasis and aetiology), outcome measures, statistical analysis and trial conduct are suboptimal by contemporary standards. The MRC study published in 1957 involved 122 bronchiectasis patients from seven centres randomised to receive penicillin $500 \mathrm{mg}$ four times a day or oxytetracycline $500 \mathrm{mg}$ four times a day or identical placebo two capsules four times a day, for 2 days per week for 1 year. ${ }^{187}$ No power calculation or formal statistical analysis was performed and no qualitative or quantitative sputum microbiology was performed. Oxytetracycline appeared most efficacious with least exacerbations requiring rescue antibiotics, a 50\% reduction in sputum purulence, markedly less days confined to bed and less days off work. Treatment with penicillin appeared to be associated with a marginal treatment response compared with placebo. A study by Cherniack et al published in 1959 involved 67 patients (45 with bronchiectasis) from a single centre. ${ }^{188}$ Patients were randomised to receive tetracycline ( 2 g/day), Penicillin G (1,600,000 units/day) Oleandomycin-Penicillin $(2 \mathrm{~g} /$ day) or identical placebo. In a subgroup analysis involving only patients with proven bronchiectasis, there were 
no statistically significant differences between the treatment groups but this may reflect a lack of power. ${ }^{188} \mathrm{~A}$ further study in 38 bronchiectasis patients looked at the use of daily oral amoxicillin $3 \mathrm{~g}$ bd over 32 weeks. In this study CTs were not performed and no reduction in exacerbations was demonstrated, but some improvement in sputum volume, diary card symptom improvement and less time away from work. ${ }^{189}$ These studies suggest that tetracycline based regimens may reduce exacerbation frequency, duration of illness and improve symptoms in patients with bronchiectasis, with penicillin based regimens being less effective.

Recent longer term antibiotic trials have only enrolled patients with bronchiectasis confirmed by high resolution CT scans. The primary clinical endpoints have concentrated on exacerbation burden with either time to next exacerbation or exacerbation frequency. There have been three recent, high quality (although small studies and heterogeneous design) RCTs performed comparing a macrolide antibiotic to placebo given over 6-12 months.

\section{Macrolides}

Wong et al studied $500 \mathrm{mg}$ three times weekly azithromycin for 6 months in 141 patients, ${ }^{190}$ Altenburg et al azithromycin $250 \mathrm{mg}$ od for 1 year in 83 patients, ${ }^{191}$ and Serisier et al $250 \mathrm{mg}$ bd erythromycin for 48 weeks in 117 patients. ${ }^{192}$ Each study used different entry criteria: 71/141 in the Wong study did not culture pathogens at baseline, and 17 cultured P. aeruginos $a^{190}$; Altenburg required at least one pathogen cultured from sputum in the previous year and $12 / 83$ had $P$. aeruginos $a^{191}$; Serisier stratified for $P$. aeruginosa at baseline and had a relatively high (41/117) number of patients with this pathogen that is associated with more severe disease. ${ }^{192}$ Wong et al required at least one exacerbation, Serisier $e t$ al at least 2, and Altenburg et al at least 3. ${ }^{190-192}$

In fact patients had a wide variation in the number of exacerbations in the previous year, but on average more than these entry criteria: Wong et al 3-4 $4^{190}$ and Altenburg et al $4-5 .{ }^{191}$ All three studies showed a significant reduction in exacerbation frequency: Wong et al 0.59 exacerbations per patient with azithromycin of 1.57 with placebo $\mathrm{P}<0.0001^{190}$; Altenburg et al median number of exacerbations 0 with azithromycin of two with placebo $\mathrm{P}<0001$, which is 0.84 per patient with azithromycin cf 2.05 with placebo ${ }^{191}$; Serisier et al 1.29 per patient with erythromycin of 1.97 with placebo $P=0.003 .{ }^{192}$ There have been four meta-analyses which have all included these three studies together with shorter studies and/or paediatric studies. ${ }^{193-196}$ The following consistent findings have been: a reduction in the number of patients who had at least one exacerbation (RR 0.55-0.7); small improvements in $\mathrm{FEV}_{1}$ occurred in 2 of the three trials and was confirmed in the meta analyses; the benefit was not dependent on microbiology; quality of life results were more variable Wu et $a l^{196} 2014$ in the five trials included in the meta analysis that used SGRQ a 5.4 fall in total score $\mathrm{P}=0.02,{ }^{196}$ but in the Guang-Ying ${ }^{195} 2014$ meta analysis that included three studies which used SGRQ the changes were not significant ${ }^{195}$ ); there were no significant increase in adverse events with macrolide, but GI side effects increase on macrolide (low dose erythromycin better tolerated than azithromycin), but in most cases not sufficient to lead to withdrawal; increased macrolide resistance occurs which was best studied by Serisier et al 2013 which showed a $27.7 \%$ increase on oropharyngeal streptococci resistance with erythromycin compared with $0.04 \%$ with placebo $\mathrm{P}<0.001 .{ }^{192}$

The results were variable at follow-up after treatment stopped, Wong suggests benefit maintained in 6 months after cessation, ${ }^{190}$
Altenburg that it is lost (in terms of exacerbations). ${ }^{191}$ Wong found treatment effects significantly greater in patients with higher SGRQ scores. ${ }^{190}$ There is some evidence favouring azithromycin. The Guang Ying et al meta analysis showed that on subgroup analysis azithromycin demonstrated lower exacerbation frequency whereas erythromycin did not. ${ }^{195}$ In addition, the benefit of erythromycin compared with placebo was more delayed in the erythromycin study compared with the other two azithromycin studies. The latter observation may however be accounted for by the higher proportion of P. aeruginosa patients in this study.

A further analysis by 16S RNA gene sequencing of paired sputum samples, baseline and end of treatment, from the BLESS trial showed a reduced abundance of $H$. influenzae, and an increased relative abundance of $P$. aeruginosa, in patients treated with erythromycin without $P$. aeruginosa at the start of the trial. ${ }^{192}$ This was not shown by routine bacteriology, but raises a concern which requires further investigation. ${ }^{197}$

\section{Inhaled/nebulised antibiotics}

In addition to the use of long term oral antibiotics, there have been several studies assessing the efficacy of long term inhaled antibiotics in bronchiectasis. Several of these have provided proof of concept data demonstrating a reduction in bacterial counts, usually at the 28 day time point. ${ }^{198} 199$ Haworth et al studied 144 bronchiectasis patients with chronic pseudomonas infection treated with Promixin (colistin) or $0.45 \%$ saline who were followed up until the first exacerbation or 6 months. ${ }^{200}$ Although the study did not reach significance in its primary endpoint of time to next exacerbation (165 days vs 111 days $\mathrm{P}=0.11$ ), this was significant in a predetermined 'compliant' population (those that took the medication for $>=80 \%$ of the time (168 vs 103 days $\mathrm{P}=0.028)$ ). This could be accurately defined because of an electronic chip on the nebuliser. In the whole group analysis they also demonstrated a significant reduction in $P$. aeruginosa colony forming unit (CFU) count at 12 weeks and a significant improvement in the SGRQ total score at 26 weeks. There was no difference in $\mathrm{FEV}_{1}$, sputum weight or adverse events. The incidence of adverse effects leading to discontinuation was low and similar in both groups.

A similar increase in time to next exacerbation was also seen in the per protocol population of a small phase 2 study of 42 bronchiectasis patients with $P$. aeruginosa with three on/off cycles of dual release inhaled ciprofloxacin. In this study, the median time to exacerbation was 134 vs 58 days $(\mathrm{P}=0.057$ modified intention to treat, 0.046 per protocol). ${ }^{199}$

Time to next exacerbation was also extended with the use of inhaled gentamicin $(80 \mathrm{mg}$ bd) compared with normal saline control in a 65 patient RCT with patients who had potentially pathogenic micro-organisms, who were treated for 1 year with a 3 month follow-up ( 61.5 vs 120 days $\mathrm{P}=0.02$ ) and the exacerbation number was also reduced in the active group $(0(0-1)$ vs 1.5 $(1-2) p<0.0001) .{ }^{201}$ Quality of life measures improved, sputum became less purulent and exercise capacity increased. Emergence of resistance did not occur, however benefits were lost quickly on stopping treatment during the 3 month follow-up. In this study patients were not masked to intervention. Although 7/32 patients in the gentamicin arm reported bronchoconstriction, this only led to withdrawal in two patients (the same as the saline arm). Further analysis of this study also documented reductions in sputum inflammatory markers (including myeloperoxidase activity, neutrophil elastase, interleukin- 8 and tumour necrosis factor-alpha). ${ }^{168}$ 
Barker and colleagues conducted two large double blinded multi-centre trials (AIR-BX1 $(\mathrm{n}=266)$ and AIR-BX2 $(\mathrm{n}=274))$ administering inhaled aztreonam $75 \mathrm{mg}$ (aztreonam for inhalation solution or AZLI) or placebo for two 4 week periods followed by a 4 week washout period after each. ${ }^{202}$ The primary end point which was change in QOL-B respiratory symptom scores (RSS) from baseline to 4 weeks was not attained in either of the studies. The secondary end points were change in QOL-B-RSS from baseline to week 12 and time to first protocol defined exacerbation to week 16. Although the QOL-B-RSS numerically increased in all groups in both studies at week 4 and week 12 , the mean change from baseline was only statistically significant in week four in the AIR-BX2 study alone. However, this change of 4.6 points although statistically significant was not clinically significant as the minimal important difference for the QOL-B-RSS is 8.0 points. The median time to first protocol defined exacerbation was reached in only the placebo group in AIR-BX1. In the AZLI treated group there were more treatment related adverse events necessitating patients to drop out. In summary, although this is a large multi centred RCT with aztreonam, there were perhaps some inherent faults with the design of the study, notably the heterogeneity of the cohorts in the study. AZLI treatment was not associated with significant clinical benefit in bronchiectasis as measured by the QOL-B-RSS and time to next exacerbation.

Most recently, Ciprofloxacin $(32.5 \mathrm{mg})$ dry powder for inhalation (DPI) twice-daily was studied in two large (RESPIRE 1 $\mathrm{n}=416$ and RESPIRE $2 \mathrm{n}=521$ ) randomised, double-blind, placebo-controlled (two active:1 placebo) trials. ${ }^{203}{ }^{204}$ A novel aspect of the studies was that 14- or 28 day on/off cycles were compared for 48 weeks. Patients had a documented history of two or more exacerbations in the previous year and had positive sputum cultures for a pathogenic species at screening. The primary end points were time to first exacerbation and frequency of exacerbations. Patients had to meet a strict definition of an exacerbation during the trial, and the number occurring were low (42.8\% in RESPIRE $1 \%$ and $58 \%$ in RESPIRE 2 of placebo patients did not have an exacerbation). In RESPIRE one ciprofloxacin DPI 14 day on/off significantly delayed time to first exacerbation versus pooled placebo (median time $>336$ days versus 186 days, HR $0.53,97.5 \%$ CI 0.36 to 0.80 , $\mathrm{P}=0.0005)$ and reduced frequency of exacerbations versus matched placebo by $39 \%$ over 48 weeks (mean number of exacerbations 0.6 vs 1.0 , incidence rate ratio $0.61,97.5 \% \mathrm{CI} 0.40$ to $0.91 \mathrm{P}=0.0061$ ). The 28 day on/off cycle results were not significant, and in RESPIRE 2, neither on/off cycle met the primary end points, although there were positive trends. A liposomal ciprofloxacin preparation for inhalation has also been studied with the complete results not published at the time of writing. The pooled results of the RESPIRE studies suggest a positive signal for response to inhaled ciprofloxacin, but the drug has not been granted marketing authorisation. Future trials must include patients more likely to exacerbate.

\section{Intravenous antibiotics}

A small study assessing the impact of cyclical 8 weekly intravenous antibiotics has also been performed in 19 patients with five or more bronchiectasis exacerbations and ill health between antibiotic courses, both defined subjectively. ${ }^{205}$ Antibiotic courses were based on sputum sensitivity. The majority of patients were not on other long term antibiotics at entry to the study (16/19). This approach demonstrated a small, statistically significant reduction in exacerbations (9.3 to 8 ) and hospital bed days (43.1 to 19.4), and improvement in incremental shuttle walk test with a proportion of patients experiencing improved health questionnaire status. However the study was subject to recall bias and the positive health effects of being in a study and having regular follow-up. There were two meta analyses done to assess the efficacy of long term antibiotics ( $>4$ weeks in bronchiectasis). While Hnin et al looked at long term antibiotics irrespective of route of delivery, ${ }^{206}$ Yang et al assessed long term inhaled antibiotics only. ${ }^{207} 18$ studies involving 1157 patients met the inclusion criteria in the metaanalysis conducted by Hnin et al. Based on the number of participants with at least one exacerbation, the meta-analysis showed significant effects in favour of the intervention (OR $0.31,95 \%$ CI 0.19 to 0.52 ; $\mathrm{P}$ value $<0.00001$ ), with events occurring in 271 per 1000 people in the intervention arm (95\% CI 126 to 385$)$ and in 546 per 1000 in the control population, based on evidence of moderate quality. A non-statistically significant reduction in hospitalisation favoured the use of prolonged antibiotics with a moderate quality grade of supporting evidence (37 per 1000 in the intervention arm (95\% CI 13 to 96) and 87 per 1000 in control (OR $0.40,95 \% \mathrm{CI} 0.14$ to 1.11 ; P value $=0.08$ ). Drug resistance developed in 36 of 220 participants taking antibiotics compared with 10 of 211 participants given placebo or standard therapy (OR $3.48,95 \%$ CI 1.20 to 10.07 ; P value $=0.02$ ), thereby translating to natural frequencies of 155 per 1000 in the intervention arm (95\% CI 59 to 346) and 50 per 1000 in the control arm. The intervention was well tolerated with no overall significant difference in withdrawal between treatment and placebo groups (OR 0.91, 95\% CI 0.56 to 1.49). Diarrhoea was commonly reported as an adverse event, particularly with an oral intervention.

The authors concluded that the available evidence shows benefit with use of prolonged antibiotics, at least halving the odds of exacerbation (with 275 fewer exacerbations per every 1000 people treated in the antibiotic arm compared with the control arm) and hospitalisation (50 fewer hospitalisations per 1000 people in the antibiotic arm compared with the control arm). However, the risk of emerging drug resistance was increased more than threefold.

Eight randomised controlled trials recruiting 539 patients were included in the meta-analysis by Yang et al. ${ }^{207}$ Long-term inhaled antibiotics showed a reduction of the sputum bacterial density (weighted mean difference $=2.85,95 \%$ CI: 1.6 to 4.09, $\mathrm{P}<0.00001)$ and augmented eradication of sputum $P$. aeruginosa $(\mathrm{OR}=6.6,95 \% \mathrm{CI}: 2.93$ to $14.86, \mathrm{P}<0.00001)$. No studies showed higher risk of $P$. aeruginosa resistance after inhaled therapy. In addition, nebulized therapy reduced the amount of patients with exacerbation $(\mathrm{OR}=0.46,95 \% \mathrm{CI}: 0.21$ to $1.00, \mathrm{P}=0.05)$. However, patients with inhaled antibiotics were more likely to suffer wheeze $(\mathrm{OR}=6.74,95 \% \mathrm{CI}$ : 2.22 to 20.52, $\mathrm{P}=0.0008)$ and bronchospasm $(\mathrm{OR}=2.84,95 \% \mathrm{CI}: 1.11$ to $7.25, \mathrm{P}=0.03)$. The authors concluded that long-term inhaled antibiotics can effectively reduce the sputum bacterial density, increase P. aeruginosa eradication and attenuate the risk of exacerbation, accompanied however by higher risk of wheeze and bronchospasm.

\section{Evidence statements}

Long term antibiotics reduce exacerbations in bronchiectasis. $(1++)$

Inhaled colistin $1 \mathrm{MU}$ twice daily delivered through the I-neb improves quality of life in bronchiectasis patients with chronic $P$. aeruginosa infection and one or more exacerbations per year. $(1+)$ 
Inhaled colistin $1 \mathrm{MU}$ twice daily delivered through the I-neb extends the time to exacerbation in treatment compliant bronchiectasis patients with chronic $P$. aeruginosa infection and one or more exacerbations per year. $(1+)$

Inhaled gentamicin increases the time to exacerbation, reduces exacerbation rate and improves quality of life in patients with bronchiectasis with chronic infection with a potentially pathogenic micro-organism and two or more exacerbations per year. $(1+)$

Azithromycin and erythromycin reduce exacerbation number, and the number of patients that had at least one exacerbation, in patients with bronchiectasis. $(1++)$

Tetracyclines may reduce exacerbation number in patients with bronchiectasis. $(2+)$

Patients recruited into the long term antibiotic RCTs had more $(\geq 3)$ exacerbations in the preceding year than defined by the inclusion criteria. $(1+)$

Inhaled aztreonam twice daily for 4 weeks is not associated with clinically significant benefit as measured by changes in the QOL-B-RSS and time to first exacerbation. Additionally, aztreonam has a higher incidence of treatment related adverse events and discontinuation secondary to adverse events. $(1+)$

Cyclical intravenous antibiotics may reduce exacerbation number and hospital bed days in patients with $\geq 5$ exacerbations and subjective ill health between exacerbations. (3)

\section{Recommendations}

> Consider long term antibiotics in patients with bronchiectasis who experience three or more exacerbations per year. (A)

> In these patients, the following are recommended (see figure 2 and appendix 2 and 3 )

\section{P. aeruginosa colonised patients}

a. Use inhaled colistin for patients with bronchiectasis and chronic $P$. aeruginosa infection. (B)

b. Consider inhaled gentamicin as a second line alternative to colistin for patients with bronchiectasis and chronic $P$. aeruginosa infection. (B)

c. Consider azithromycin or erythromycin as an alternative (eg, if a patient does not tolerate inhaled antibiotics) to an inhaled antibiotic for patients with bronchiectasis and chronic $P$. aeruginosa infection. (B)

d. Consider azithromycin or erythromycin as an additive treatment to an inhaled antibiotic for patients with bronchiectasis and chronic $P$. aeruginosa infection who have a high exacerbation frequency. (D)

\section{Non- P. aeruginosa colonised patients}

e. Use azithromycin or erythromycin for patient with bronchiectasis. (A)

f. Consider inhaled gentamicin as a second line alternative to azithromycin or erythromycin. (B)

g. Consider doxycycline as an alternative in patients intolerant of macrolides or in whom they are ineffective. (C)

\section{Good practice points}

$\checkmark$ Antimicrobial stewardship is important

$\checkmark \quad$ Prior to starting long term macrolides, for safety reasons: 1) ensure no active NTM infection with at least one negative respiratory NTM culture; 2) use with caution if the patient has significant hearing loss needing hearing aids or significant balance issues. $\checkmark$ Prior to starting long term inhaled aminoglycosides, for safety reasons: 1) avoid using if creatinine clearance $<30 \mathrm{~mL} / \mathrm{min}$; 2) use with caution if the patient has significant hearing loss needing hearing aids or significant balance issues; 3) avoid concomitant nephrotoxic medications.

$\checkmark$ Counsel patients about potential major side effects with long term antibiotics, and to seek urgent attention if these develop.

a. Review the patient's culture and mycobacterial status, optimise airway clearance and treat other associated conditions before starting long term antibiotics.

b. Prophylactic antibiotics should be only started by respiratory specialists.

c. Review patients on long term antibiotics 6 monthly with assessment of efficacy, toxicity and continuing need. Monitor sputum culture and sensitivity regularly, although in vitro resistance may not affect clinical efficacy.

$\checkmark$ As adverse event frequency of azithromycin is likely to be dose related, $250 \mathrm{mg} 3 \mathrm{x} /$ week is a pragmatic starting dose which can then be increased according to clinical response and adverse events.

$\checkmark$ Thresholds for long term treatment may reduce if the patient is symptomatic between exacerbations and/or the exacerbations respond poorly to treatment and/orthe patient is at high risk of severe exacerbation for example, immunosuppressed.

$\checkmark$ Long term antibiotic choice is complex and has to take into account factors such as tolerance, allergies and sensitivity, therefore in some circumstances, other long term antibiotic regimens may be appropriate (see appendix 3).

$\checkmark$ Perform a suitable challenge test when stable before starting inhaled antibiotics (see appendix 2)

$\checkmark$ Consider cyclical IV antibiotics in patients with repeated infections ( $\geq 5 /$ year) despite other treatments.

$\checkmark$ Alternative inhaled/nebulised agents may become licensed as international studies are completed.

$\checkmark$ For patients receiving long term prophylactic oral antibiotics, the preferred option is to remain on the same antibiotic as opposed to monthly rotation of antibiotics. If there is a subsequent lack of efficacy, the antibiotic can be changed guided by sensitivity results.

\section{Research recommendation}

Long term randomised controlled trials of oral and inhaled antibiotics are needed to assess their efficacy and safety in patients with bronchiectasis who have frequent respiratory tract infections with recurrent $P$. aeruginosa infection or other potential pathogenic micro-organisms.

\section{Does long term bronchodilator treatment improve outcomes for patients with bronchiectasis?}

Patients with bronchiectasis frequently have airflow obstruction and more than $60 \%$ of bronchiectasis patients have daily symptoms of breathlessness. ${ }^{120}$ There is limited supporting evidence for the use of bronchodilators, although both beta-2-agonists and anticholinergic bronchodilators are commonly used in clinical practice. ${ }^{166}$

\section{Long acting Beta-2-agonists}

A systematic review published in 2014 identified only one eligible randomised controlled trial of long acting beta-2-agonists. $^{208}$ This included 40 patients with a primary outcome of health related quality of life. It indicated improved quality of life with the use of budesonide/formoterol versus budesonide 
alone. The Cochrane review identified significant methodological issues with this trial including inadequate and incorrectly reported blinding and inadequate reporting of the data. ${ }^{208}$

\section{Long acting anticholinergic agents}

There are no randomised controlled trials of long acting muscarinic antagonists in bronchiectasis. ${ }^{209}$ Although studies suggest that some patients with bronchiectasis will have significant reversibility to anti-cholinergic bronchodilators, there is no evidence to establish that reversibility is a requirement for benefit. $^{210}$

\section{Short acting bronchodilators}

There are no randomised controlled trials to demonstrate if short acting bronchodilators are beneficial in bronchiectasis. ${ }^{211}$

As there are no large randomised controlled trials of bronchodilators in bronchiectasis there are limited data on the safety of these agents. Data from COPD and asthma, however, suggests that they have an acceptable safety profile.

\section{Evidence statement}

Long acting beta-2-agonists may be beneficial in patients with symptoms of breathlessness. (4)

Long acting anti-cholinergics in bronchiectasis may be beneficial in patients with symptoms of breathlessness. (4)

No evidence was identified to support the use of short acting beta-2-agonists. (4)

Bronchodilator treatment may include short or long acting beta-agonists, short or long acting anti-cholinergic bronchodilators, or combined bronchodilators. There is no evidence to guide which of these is the optimal strategy in bronchiectasis (4)

\section{Recommendations}

$>$ Use of bronchodilators in patients with bronchiectasis and co-existing COPD or asthma should follow the guideline recommendations for COPD or asthma. (D)

$>$ Offer a trial of long acting bronchodilator therapy in patients with symptoms of significant breathlessness. (D)

> Reversibility testing to beta-2-agonists or anticholinergic bronchodilators may help to identify patients with co-existing asthma but there is no evidence to suggest that a response is required in order to benefit from bronchodilators. (D)

\section{Pulmonary rehabilitation \\ Evidence summary}

A recent cross-sectional study by Bradley et al assessed the physical activity of participants with bronchiectasis $(n-=63){ }^{212}$ Only $11 \%$ of participants met the recommended physical activity guidelines of $\geq 150 \mathrm{~min}$ of at least moderate activity per week. ${ }^{213}$ There are four randomised controlled trials (RCTs) which investigate the effects of pulmonary rehabilitation (PR) in bronchiectasis. ${ }^{150}{ }^{214-216}$ Mandal et al (2012) conducted a pilot RCT $(\mathrm{n}=30)$ comparing an 8 week PR programme plus respiratory physiotherapy with respiratory physiotherapy alone. ${ }^{215}$ Respiratory physiotherapy was carried out twice daily using the oscillatory positive expiratory pressure (PEP) device the Acapella Choice. Outcome measures were the incremental shuttle walk test (ISWT), the endurance shuttle walk test (ESWT), the Leicester Cough Questionnaire (LCQ) and the St George's Respiratory Questionnaire (SGRQ). After 8 weeks there was a significant improvement in all outcome measures in the intervention group compared with the control group. The mean difference in the ISWT, LCQ and SGRQ were all clinically important as they exceeded the mean clinically important difference (MCID) for each outcome measure. These benefits were maintained at 20 weeks (12 weeks post end of PR programme). Participants in the PR group were encouraged to continue with their exercise programme after completion of the 8 week programme and were offered free gym membership. However, no information was collected on the intensity or frequency of exercise between weeks $8-20$ and so it is difficult to draw any conclusions on the maintenance effect. Although this was a small trial, it was sufficiently powered to detect a mean difference in the primary endpoint (ISWT).

Lee et al (2014) conducted a well-powered RCT $(\mathrm{n}=85)$ comparing an 8 week supervised exercise programme with a control group who received no intervention. ${ }^{150}$ Participants were included if they reported dyspnoea on exertion (Modified Medical Research Council Dyspnoea (mMRC) score of $\geq 1$ ). A mMRC score of 1 equates to getting short of breath when hurrying on level ground or walking up a slight hill. Primary outcomes were exercise capacity (ISWT and 6 min walking distance (6MWD)) and health related quality of life Chronic Respiratory Disease Questionnaire (CRDQ). Secondary outcomes were cough-related quality of life (LCQ) and psychological symptoms (Hospital Anxiety and Depression Scale (HADS)). There was a significant improvement in exercise capacity in the intervention group compared with the control group but these improvements were not sustained at 6 or 12 months. There were also significant improvements in the dyspnoea and fatigue domains of the CRDQ but not the domains of emotional function or mastery. Exercise training did not have an effect on cough-related quality of life or anxiety and depression. There was a statistically significant reduction in the frequency of acute exacerbations in the exercise group (median 1 (IQR1-3)) compared with the control group (2 (1-3)) over 12 months $(\mathrm{P}=0.012)$. There was also a statistically significant longer time to first exacerbation in the exercise group of 8 months (95\% CI 7 to 9 months) compared with the control group (6 months (95\% CI 5 to 7 months) $\mathrm{P}=0.047)$. However, these results should be interpreted with caution as a significant number of participants $(30 / 85)$ that did not complete the 12 month follow-up.

The most recent randomised controlled trial by Olveira et al (2015) $(n=30)$ aimed to assess the effect of a 12 week PR programme versus a PR programme plus a hyperproteic oral nutritional supplement. ${ }^{216}$ Exercise capacity was not a reported outcome measure. Muscle strength measured using mean handgrip dynamometry was significantly improved in both groups at 12 and 24 weeks. Health-related quality of life assessed using the Quality of Life - Bronchiectasis questionnaire QOL-B Physical Functioning scale significantly improved in the PR plus nutritional supplement group but not in the PR alone group. However, these results should be interpreted with caution for two main reasons. First, there was a large difference in the baseline QOL-B score of the two groups. Although this was not a statistically significant difference, the mean QOL-B score of the $\mathrm{PR}$ alone group was more than 12 points lower than the PR plus nutritional supplement group at baseline. In addition, between group statistical analysis was not performed.

An uncontrolled, retrospective study by Van Zeller et $a l^{217}$ (2012) assessed the effect of a 12 week PR programme on 41 participants with bronchiectasis. ${ }^{217}$ The exercise component consisted primarily of cycle ergometer exercise with additional upper limb and quadriceps training. This does not reflect current UK clinical practice where PR programmes usually include 
a walking component in order to improve walking function. There was no significant change in 6MWD at the end of the programme. These results are in direct contrast to the aforementioned RCTs which all showed that exercise had a clinically significant effect on exercise capacity. ${ }^{150} 214215$

A larger $(n=135)$ and more recent retrospective study evaluated the effect of a 3 week inpatient PR programme on individuals with bronchiectasis. ${ }^{218}$ This PR programme had a statistically significant effect on 6MWD which improved by $35 \pm 43 \mathrm{~m}$ $(\mathrm{P}<0.0001)$. Health-related quality of life was measured using the EuroQol visual analogue scale (EQ-VAS) and improved by $15 \pm 12(\mathrm{P}<0.0001)$. Dyspnoea was measured with the Baseline/ Transition Dyspnoea Index (BDI/TDI) and this also improved after the programme from $7.3 \pm 2.9$ to $4 \pm 2.6$ (no $\mathrm{p}$ value given). Regression analyses revealed that male gender, baseline $\mathrm{FEV}_{1}$ / $\mathrm{VC}<70 \%$ and $>2$ exacerbations in the previous year were independent predictors of improvement in exercise capacity and health-related quality of life. Although this is a retrospective and uncontrolled study, it does reveal that patients with more severe bronchiectatic disease also benefit from inclusion in a PR programme.

There is currently no evidence assessing the incidence of cross infection of respiratory pathogens between individuals with bronchiectasis in the group exercise setting.

In summary, there is strong evidence from a number of trials to show that PR increases exercise capacity and can improve health-related quality of life in bronchiectasis. One RCT has also shown that PR reduces the frequency of acute exacerbations over a 12 month period and increases the time to first exacerbation, although there are significant methodological issues regarding dropouts. $^{150}$

\section{Inspiratory muscle training}

Two studies investigate the effects of inspiratory muscle training (IMT). ${ }^{214} 219$ The study by Liaw et $a l^{219}$ found that an 8 week home-based IMT training programme resulted in a significant difference in inspiratory and expiratory muscle strength in the intervention group when compared with the control. However, there were no significant between group differences in any other measure including pulmonary function, quality of life or walking capacity. This study was at high risk of bias due to significant differences between groups at baseline in some measures of pulmonary function and also age. There were a relatively high number of drop outs and intention to treat analysis was not employed. There were 19 participants in each group but six participants dropped out in each group and therefore only 13 participants in each group were included for final analysis. A final consideration is that the participants were not blinded as the control group did not receive any form of sham IMT.

The study by Newall et $a l^{214}$ was well-conducted but had a small number of participants $(n=32) .{ }^{214}$ This study had three groups: PR plus IMT, PR plus sham IMT and a control group which received no intervention. Exercise capacity increased significantly in both the PR-SHAM and PR-IMT groups. HRQOL was significantly better in the PR-IMT group than the PR-SHAM. After 3 months, exercise capacity had declined significantly in the PR-SHAM group but had been maintained in the PR-IMT group. The findings of this study suggest that there is no additional advantage of simultaneous IMT with PR to increase exercise capacity. However, IMT may be important in prolonging the benefit of the training effects.

\section{Field walking tests}

Research has now been conducted to support the use of the 6MWT and ISWT as reliable and responsive measures of exercise capacity in the bronchiectasis population (Lee et al). ${ }^{220}$ This study also found that there was a small learning effect in both measures at baseline and it is therefore recommended that two of each test are performed to accurately assess exercise capacity. In addition, a minimal important difference has been estimated for the 6MWT $(25 \mathrm{~m})$ and ISWT $(35 \mathrm{~m})$ in bronchiectasis (Lee et al). ${ }^{221}$ Therefore, these are suitable outcome measures to assess exercise capacity before and after PR in the bronchiectasis population.

\section{Evidence statements}

Pulmonary rehabilitation increases exercise capacity and can improve quality of life in individuals with bronchiectasis. $(1+)$

Pulmonary rehabilitation can reduce frequency of exacerbations over a 12 month period and can increase the time to first exacerbation. (1-)

IMT, when used in conjunction with pulmonary rehabilitation, can enhance the longevity of the training effects. $(1+)$

IMT in isolation does not increase exercise capacity or quality of life in individuals with bronchiectasis. (1-)

$6 \mathrm{MWT}$ and ISWT are reliable and responsive outcome measures for use in bronchiectasis to evaluate exercise capacity pre and post pulmonary rehabilitation. (3)

There is currently no evidence assessing the incidence of cross infection of respiratory pathogens between individuals with bronchiectasis in the group exercise setting.

\section{Recommendations}

> Offer pulmonary rehabilitation to individuals who are functionally limited by shortness of breath (Modified Medical Research Council (MMRC) Dyspnoea Scale $\geq 1$ ). (B)

$>$ Consider the use of inspiratory muscle training in conjunction with conventional pulmonary rehabilitation to enhance the maintenance of the training effect. (B)

\section{Good practice points}

$\checkmark$ Educate all individuals with bronchiectasis on the importance of an exercise training programme.

$\checkmark$ Consider the 6MWT and/or the ISWT when evaluating exercise capacity pre/post pulmonary rehabilitation in bronchiectasis. Prior to this, practice tests should be carried out to eliminate any learning effect.

$\checkmark$ Pulmonary rehabilitation providers should offer education sessions tailored to the needs of individuals with bronchiectasis (eg, airway clearance techniques, the pathophysiology of bronchiectasis and relevant inhaled therapy).

$\checkmark$ Pulmonary rehabilitation exercise and education sessions should be provided by appropriately qualified healthcare practitioners.

Further information on Pulmonary rehabilitation is provided in the BTS Quality Standards for Pulmonary rehabilitation (https://www.brit-thoracic.org.uk/standards-of-care/qualitystandards/bts-pulmonary-rehabilitation-quality-standards/)

\section{Research recommendation}

The role of education, self management plans and who delivers the pulmonary rehabilitation needs to be explored.

The role of pulmonary rehabilitation after exacerbations requiring hospital admission needs to be explored. 
The incidence of cross-infection of respiratory pathogens in the group exercise setting should be investigated in the bronchiectasis population.

\section{What is the role of surgery in managing bronchiectasis?}

Multiple case series of surgical resection for bronchiectasis are available. ${ }^{22-237}$ A Cochrane review concluded there are no high quality randomised control studies and the benefits of surgery over conservative management were unclear. It is also likely there is a reporting bias for the available case series. ${ }^{238}$

Generally surgery should not be considered until there is optimisation of medical management. With this caveat the consistency of available evidence supports a role for surgery in bronchiectasis in selected patients.

The indications for surgical resection in bronchiectasis include

o persistent symptoms despite up to a year of comprehensive medical treatment,

o exacerbations that are either severe or frequent and interfere with social/professional life,

- recurrent refractory or massive haemoptysis,

- post obstruction bronchiectasis distal to tumours

- localised severely damaged lobe/segment that may be a source of sepsis that left in situ may lead to extension of lung damage.

Surgery is generally applied when there is focal disease in the presence of the above clinical scenarios. Therefore the population both requiring and suitable for surgery is likely to be small. Notably many series reported to date included patients with a lower mean age than seen in many clinics, ranging from 30 to 48 years of age. ${ }^{222} 225239$ The median duration of symptoms prior to surgery in the reported series was usually prolonged e.g. 6 years. $^{225}$

A variety of operative techniques have been described. In experienced hands video assisted thoracoscopic (VAT) performs as well as open surgery 231233239 and may be associated with lower rates of complications such as blood loss and shorter hospitalisations. ${ }^{233}$ There is however an intraoperative conversion rate from VATS to open surgery of up to $12 \%$. $^{233}$ The resection limits range from segmentectomy to pneumonectomy but the available literature does not provide robust decision making tools to aid decision making on the optimal resection limits in day to day practice. $^{222225231233239}$

With this in mind pre-surgical safety assessment should be undertaken to define the expected amount of residual lung, underlying cardiopulmonary reserve and anticipated overall risks. Pulmonary rehabilitation and optimisation of nutrition are important aspects with which respiratory physicians can support their patients.

In the available series the 60 day mortality outcomes ranged from 0\%-11\%. 224232237239 Common post-operative complications included wound infection, empyema, systemic sepsis, post thoracotomy pain, prolonged air leak and recurrence of bronchiectasis. ${ }^{224-226} 234235$ Early morbidity rates ranged from $13 \%$ to $24 \% 222225232235239$ with wound infection reported as the most common complication (6\%) in one large series of 277 patients. $^{240}$

For long term complications the recurrence rate is likely to depend on the completeness of resection and underlying aetiology of bronchiectasis. Perceived complete resection is associated with significantly better long term outcomes than cases where incomplete resection has occurred. 223225232239240 In one small case series of 31 patients, the mean time to recurrence of symptoms was 34 months. ${ }^{232}$ An immunocompromised status, persistent infection with $P$. aeruginosa and the extent of residual bronchiectasis after surgery have been associated with a significantly shorter recurrence-free interval. ${ }^{232}$ The primary cause of bronchiectasis may influence post-surgical outcomes with likely better outcomes after post obstructive bronchiectasis and poorer outcomes in immunodeficiency. ${ }^{232}$ There are however limited data to guide clinicians in defining risk benefit ratios. ${ }^{229} 238$ Even when the primary cause of bronchiectasis persists, successful outcomes are reported. Surgery can be successful in primary ciliary dyskinesia. $^{226}$

Freedom from symptoms of bronchiectasis have reported as high as $61 \%-84 \%$ when followed up from between 1 to 5 years or more post-operatively. 224225234

\section{Evidence statement}

Surgery may be successful in bronchiectasis in reducing exacerbations or treating haemoptysis. (3)

The ideal candidate(s), timing for surgery and operative approach remain ill-defined. (3)

Video assisted thoracoscopic technique can provide outcomes at least as successful as open surgery. (3)

Improvements in bronchiectasis symptoms can be seen after surgery for bilateral disease. (3)

\section{Recommendations}

$>$ Consider lung resection in patients with localised disease whose symptoms are not controlled by medical treatment optimised by a bronchiectasis specialist. (D)

> Offer multidisciplinary assessment, including a bronchiectasis physician, a thoracic surgeon and an experienced anaesthetist, of suitability for surgery and pre-operative assessment of cardiopulmonary reserve post resection. (D)

\section{Good practice point}

$\checkmark$ Consider nutritional support and pre-operative pulmonary rehabilitation before surgical referral.

\section{Lung transplantation for bronchiectasis}

Lung transplantation is an effective therapy for bronchiectasis when maximal medical therapy is failing. Lung transplantation is generally reserved for those with diffuse bilateral disease and bilateral transplantation is then performed. There are however reports of single lung transplantation with contralateral pneumonectomy. Generally lung transplantation is felt indicated where survival is anticipated at $50 \%$ at 2 years without lung transplantation. ${ }^{241}$ International guidelines note the risks of transplantation in general appear to increase with age and generally patients above the age of 65 are felt to have an unfavourable risk: benefit mortality ratio. ${ }^{241}$

Internationally bronchiectasis is reported as a rare indication for lung transplantation representing less than 5\% of all bilateral lung transplantations recorded in the international registry. Overall less than 1200 transplant procedures for bronchiectasis were recorded in the international registry as compared with 1280 for pulmonary hypertension, 6862 for cystic fibrosis and 13672 for COPD (https://www.ishlt.org/registries/slides.asp? slides=heartLungRegistry accessed Aug 2015).

The available case series focus on lung transplantation for adults with bronchiectasis with no significant data reported for paediatric transplantation. While collectively less than 200 patients, these case series report good outcomes following transplantation. In a case series of 22 patients transplanted in the 
UK between 1988 and 2001 undergoing bilateral lung transplantation either as bilateral sequential single-lung transplants (BSSLTX), heart-lung transplants (HLTX) or single-lung transplants (SLTX) the reported outcomes included lung function and mortality data. ${ }^{242}$ Overall the 1 year Kaplan-Meier survival for all patients was $68 \%$ (95\% CI, $54 \%$ to $91 \%$ ), with the 5 year survival reaching $62 \%$ (95\% CI, $41 \%$ to $83 \%)$. In those undergoing SLTX for bronchiectasis the 1 year survival was lower at $57 \%$ (95\% CI, 20\% to 94\%) compared with those receiving 2 lungs $73 \%$ (95\% CI, $51 \%$ to 96\%). At 6 months, mean forced expiratory volume in $1 \mathrm{~s}$ was $73 \%$ predicted (range, 58\%-97\%), and mean forced vital capacity was $68 \%$ predicted (range, 53\%-94\%) after receiving two lungs $(n=10)$.

A small single centre case series of 37 patients from Cambridge, UK reported transplantation outcomes in those with bronchiectasis due to immunodeficiency syndromes $(\mathrm{n}=5$, predominantly antibody deficiency patients on replacement therapy) compared with other causes of bronchiectasis $(n=32){ }^{243}$ The actuarial survival was comparable between the two groups. The 1 year survival was $81 \%$ in the bronchiectasis group and $80 \%$ in bronchiectasis-antibody deficiency recipients. Functional outcomes following transplant were good with the $\mathrm{FEV}_{1}$ (mean +/-SD) at 1 year post transplant being $83.7 \%+/-24.2 \%$ in the bronchiectasis group and $83.0 \%+/-30.4 \%$ in the bronchiectasis-antibody deficiency recipients.

A single centre experience of 17 bronchiectasis patients from Madrid, Spain reported that in a series of 171 patients, 44 (26\%) were transplanted for suppurative lung diseases. ${ }^{244}$ Of these 27 had cystic fibrosis (61\%) and 17 (39\%) were classified as bronchiectasis. The reported survival of cystic fibrosis patients was $85 \%$ at 1 year and $45 \%$ at 5 years while that of bronchiectasis patients demonstrated an initially higher mortality rate (survival $70 \%$ at 1 year) but similar long term survival (54\% at 5 years). P. aeruginosa infection in pre-transplant recipients was common but not invariable. No clear association was seen between pre transplant $P$. aeruginos $a$ and poorer post transplant outcomes, though the study was unlikely to be powered. Persistence of the pre-transplant infecting organism was common at 1 year following transplantation. ${ }^{244}$

One study of over 1000 patients in the UK waiting for lung transplantation included adults with cystic fibrosis $(n=430)$, bronchiectasis $(n=123)$, pulmonary hypertension $(n=74)$, diffuse parenchymal lung disease $(n=564)$, chronic obstructive pulmonary disease (COPD $n=647)$ and miscellaneous indications $(n=159)$. The proportion of patients in each group who died while on an active transplant list listed varied significantly (respectively $37 \%$, $48 \%, 41 \%, 49 \%, 19 \%, 38 \%)$. Hence these data demonstrate that bronchiectasis has one of the highest 'on-list' mortality rates with up to $60 \%$ mortality on list at 2 years. ${ }^{245}$

Furthermore in the 54 of 123 bronchiectasis patients on the waiting list that survived to transplantation, median survival was 3001 days which was better post-transplant survival rates than more common indications for transplantation such as COPD ( $\mathrm{n}=483$ transplanted, median survival 1795 days) and cystic fibrosis ( $n=234,2436$ days). ${ }^{245}$

Consistent with international guidelines, those transplanted had severe disease (mean FEV $26 \%$ predicted) and were younger than the median age of patients reported in the UK national audit of outpatient bronchiectasis with average age at transplantation 49.7 years. ${ }^{245}$

\section{Evidence statement}

As stated in international Lung transplantation guidelines, post-transplant morbidity and mortality increase with age and hence transplantation is in general reserved for those aged 65 years or less. (3)

In selected patients with bronchiectasis, lung transplantation improves quality of life and is associated with post-transplant survival of over $60 \%$ at 5 years. (3)

Bronchiectasis patients have poor outcomes on lung transplant waiting lists with mortality rates of up to $60 \%$ at 2 years. (3)

The majority of transplantation procedures performed for bronchiectasis are bilateral lung transplants in patients with severely impaired quality of life or rapid deterioration. (3)

\section{Recommendation}

$>$ Consider transplant referral in bronchiectasis patients aged 65 years or less if the $\mathrm{FEV}_{1}$ is $<30 \%$ with significant clinical instability or if there is a rapid progressive respiratory deterioration despite optimal medical management. (D)

> Consider earlier transplant referral in bronchiectasis patients with poor lung function and the following additional factors: massive haemoptysis, severe secondary pulmonary hypertension, ICU admissions or respiratory failure (particularly if requiring NIV). (D)

\section{Good practice points}

$\checkmark$ Discuss appropriate patients with a transplant centre prior to formal referral.

$\checkmark$ Optimise management of comorbidities such as osteoporosis and maintaining physical condition through pulmonary rehabilitation prior to transplant.

\section{What is the role of influenza and pneumococcal vaccination in management of bronchiectasis}

\section{Influenza vaccination}

There are no randomised controlled trials evaluating the impact of annual influenza vaccination on respiratory exacerbations and pulmonary decline in adults with bronchiectasis. ${ }^{246}$ Evidence for the potential benefits of influenza vaccination may be derived from studies of patients with chronic obstructive pulmonary disease (COPD), some of whom will also have bronchiectasis. In a systematic review of influenza immunisation in COPD, administration of inactivated influenza vaccines in placebo-controlled randomised controlled trials was associated with reduced late exacerbations (Weighted Mean Difference $-0.39,95 \%$ CI -0.61 to -018$)$ and laboratory confirmed influenza infection (Odds Ratio-0.19, 95\% CI - 0.07 to - 048) in COPD patients. ${ }^{247}$ Observed reduction in exacerbations of COPD was due to fewer influenza infections occurring 3 weeks or more after influenza immunisation. Vaccination did not cause an increase in short term exacerbation (within 2 weeks of immunisation) but was associated with a transient increase in injection site pain and redness. Influenza vaccination had no impact on hospitalisation, mortality, lung function and exercise tolerance however the number of studies assessing some parameters (hospital admissions, death rates) was very small and hence type two statistical error cannot be excluded. Although there are few randomised controlled trials of influenza vaccination in COPD, the results of such studies are consistent with those observed in large retrospective cohort studies. ${ }^{247}$

\section{Pneumococcal vaccination}

There is limited evidence supporting the use of 23 valent pneumococcal vaccine to prevent exacerbations of bronchiectasis. ${ }^{248}$ One randomised controlled trial comparing pneumococcal and influenza vaccination versus influenza vaccination alone in 
patients with chronic lung disease including COPD and bronchiectasis showed that patients given the 23 valent pneumococcal vaccine had fewer exacerbations. ${ }^{249}$ The NNV was $6(95 \%$ CI 4 to 32) over 2 years. No difference between groups was observed for pneumonia incidence, and pulmonary function test data were not reported. The limitations of this study include open label design, small number of patients with bronchiectasis enrolled $(n=20)$, lack of microbiological evidence for pneumococcal infection and failure to document severity of chronic lung disease.

Randomised controlled trials show that 23 valent pneumococcal vaccination can prevent all cause pneumonia (OR 0.72 $95 \%$ CI 0.56 to 0.93$).^{250}$ There is however marked statistical heterogeneity between studies, according to study site (high income $\mathrm{v}$ low income countries), population (healthy adults $\mathrm{v}$ those with chronic medical illnesses) and the pneumococcal vaccine used ( 14 valent $v 23$ valent). The 23 valent pneumococcal vaccine was effective against all cause pneumonia in low income countries (OR 0.54 (5\% CI 0.43 to 0.67 ) but in high income countries, studies in healthy adults and in those with chronic medical illnesses were negative. ${ }^{250}$ The 23 valent pneumococcal vaccine is still offered in the UK, based on the evidence that, for adults between the ages of 65-74 with chronic lung disease, the 23 valent polysaccharide pneumococcal vaccine continues to provide short term protection against invasive pneumococcal disease and remains cost effective. ${ }^{251} 252$

The role of protein conjugate pneumococcal vaccination is contentious. There is no evidence as to the efficacy of protein conjugate pneumococcal vaccination in patients with bronchiectasis.

\section{Evidence statements}

The potential benefit of influenza vaccination in reducing exacerbations and influenza infection is derived from studies of individuals with COPD, some of whom will also have bronchiectasis. In the absence of direct evidence, influenza vaccination can be justified based on individual patient needs (presence of COPD, immune deficiency, nursing home residence, patient preference and expert opinion) and adherence to national guidelines. (4)

The 23 valent polysaccharide pneumococcal vaccination with influenza immunisation may reduce exacerbations of bronchiectasis. (1-)

Meta-analysis of RCTs supported the use of 23 valent pneumococcal vaccine in reducing the rate of all cause pneumonia in healthy adults and those with chronic illnesses although there was marked statistical heterogeneity. $(1+)$

\section{Recommendations}

> Offer annual influenza immunisation to all patients with bronchiectasis. (D)

> Offer polysaccharide pneumococcal vaccination to all patients with bronchiectasis. (D)

\section{Good practice point}

$\checkmark$ Consider influenza vaccination in household contacts of patients with immune deficiency and bronchiectasis to reduce the risks of secondary transmission.

$\checkmark$ Consider use of 13 valent protein conjugate pneumococcal vaccine in patients with bronchiectasis who do not have an appropriate serological response to standard polysaccharide vaccine (23 valent carbohydrate Pneumococcal vaccine).

\section{Treatment of respiratory failure}

A study of survival of 48 patients in the intensive care unit (ICU) where one of the interventions was NIV $(n=13,27 \%)$ found cumulative mortality of $19 \%(n=9)$ for a first admission to ICU for respiratory failure in patients with bilateral non-CF bronchiectasis and $40 \%(n=19)$ at 1 year. ${ }^{253}$ The actuarial survival rate at 1 year was $60 \%$. Intubation requirement was associated with reduced survival in univariate analysis, suggesting that NIV may be advantageous. Multivariate analysis found that age $>65$ years and long-term oxygen therapy were independent factors predicting reduced survival. Survival was not increased for those treated with long-term non-invasive positive pressure ventilation. A retrospective analysis of patients treated with non-invasive $(n=31)$ versus invasive $(n=26)$ ventilation for respiratory failure reported a $32 \%$ failure rate with NIV for progression to IV or death. ${ }^{254}$ The overall in hospital mortality was around $25 \%$, not different between NIV and IV, and predicted by APACHE score. Bronchiectasis in the context of COPD may increase the duration of ICU stay (median 14 vs 9 days) but did not influence mortality in a study of 93 patients with COPD on invasive ventilation, $31 \%$ of whom had radiological bronchiectasis. ${ }^{255}$

A study of the outcome of domiciliary NIV noted that the response to NIV in this group of patients $(n=13)$ was disappointing. ${ }^{256}$ The patient group had more severe hypoxaemia and hypercapnia at the start, suggesting that NIV was introduced later in the natural history of the disease than in previous studies. The probability of continuing NIV after 2 years in the bronchiectasis group was $<20 \%$ and most patients became increasingly more ventilator dependent with time. However, all reported improvement in quality of sleep and in levels of daytime activity. Eight considered that the improvement in their respiratory status outweighed the discomfort due to NIV.

A retrospective study of 16 patients with severe diffuse bronchiectasis investigating NIV as rescue therapy for nocturnal home use with daytime oxygen found a significant increase in $\mathrm{FEV}_{1} 12$ months after initiation of NIV. In patients alive after 24 months there was a significant decrease in the length of hospital admissions. $^{257}$ This could be a reflection of the three deaths being the high intensity users.

In a study which compared patients with bronchiectasis treated with NIV with those treated with long-term oxygen therapy only, differences were found between the two groups. In the year before NIV was commenced, mean/SD length of time in hospital for the NIV and long-term oxygen therapy groups was $48+/-55$ and 5+/-8 days, respectively; the year following home NIV the length of time in hospital for the two groups was $10+/-31$ and $9+/-16$ days, respectively. ${ }^{258}$ In contrast, a further study did not find a reduction in hospital days with the instigation of non-invasive positive pressure ventilation in patients with bronchiectasis. $^{259}$

\section{Evidence statements}

There are no specific studies of LTOT for respiratory failure in bronchiectasis, but expert opinion based on data in COPD is that this is likely to be a beneficial intervention. (4)

NIV may lead to a reduction in hospitalisation days in patients with bronchiectasis and hypercapnic respiratory failure however the proportion of patients remaining on NIV at 2 years is low. (3)

\section{Recommendations}

> Consider long term oxygen therapy for patients with bronchiectasis and respiratory failure, using the same eligibility criteria as for COPD. (D) 
> Consider domiciliary non-invasive ventilation with humidification for patients with bronchiectasis and respiratory failure associated with hypercapnia, especially where this is associated with symptoms or recurrent hospitalisation. (D)

\section{Bronchiectasis and other treatments: cough suppression, nutritional supplements, complementary therapy/ homeopathy, supplemental treatments}

The majority of studies of alternative therapies and bronchiectasis are mainly observational with small patient numbers. There is a lack of randomised controlled trials.

A small $(n=35)$ randomised, partially blinded study (over 8 weeks) involving three groups, on the role of self-administered acupressure to reduce the symptoms of bronchiectasis and improve QOL. ${ }^{260}$ The three groups were standard care + self administered acupressure $(n=11)$, standard care + shamacupressure $(n=11)$ (not applied to real acu-points) and standard care alone $(n=13)$. All aged 40-70 years (sex not identified). Originally 49 enrolled, 12 dropped out by week 4,2 by week 8 .

The findings showed that after 8 weeks of self-administered acupressure seemed to be useful in reducing the effects of bronchiectasis on patients' daily activities, however, the authors conceded that sham acupressure administered for the same amount of time was capable of improving some of the symptoms of bronchiectasis. A Cochrane review, Singing for children and adults with bronchiectasis, found no eligible trials, therefore, no data were available for analysis. ${ }^{261}$ A review ${ }^{262}$ and a controlled study ${ }^{263}$ around vitamin D both concluded that vitamin D deficiency was common in bronchiectasis, however, there were no RCTs assessing vitamin D. A case control study on a small number of patients $(n=34$ with bronchiectasis, $n=29$ without) found that the patients with bronchiectasis had significantly lower serum zinc level than the control group, however, there were no trials to evaluate the prophylactic or therapeutic use of zinc supplements. ${ }^{264}$

\section{Evidence statement}

Alternative treatments such as complementary therapy/homeopathy and supplemental treatments have limited evidence to support their use.

\section{Recommendation}

$>$ Do not routinely recommend alternative treatments (for example cough suppression, nutritional supplementation, complementary therapy/homeopathy, supplemental treatments) as part of the management of patients with bronchiectasis. (D)

\section{Good practice point}

$\checkmark$ Record patient's weight and BMI at each clinic appointment.

\section{Research recommendations}

Further interventional/randomised controlled trials needed to establish the role of any alternative therapies in the management of bronchiectasis.

Studies assessing the benefits of nutritional supplementation in patients with bronchiectasis should be undertaken.

\section{SECTION 10}

Do pathogens have an impact on prognosis in bronchiectasis? In UK studies, the most frequent pathogen isolated from traditional culture in bronchiectasis is Haemophilus influenzae (20\%40\%), followed typically by $P$. aeruginosa (10\%-30\%), Moraxella catarrhalis, Streptococcus pneumoniae, Staphylococcus aureus and enterobacteriaceae. ${ }^{68120} 265266$ Patients with NTM account for $1 \%-10 \%$ of cases. ${ }^{267} 268$

There is a clear and consistent association between the presence of $P$. aeruginosa and poor outcome. All studies that have examined this outcome conclude that $P$. aeruginosa is associated with a three-fold or greater increased risk of mortality, even after adjustment for potential confounders. 120266269270

Patients with $P$. aeruginosa are also more likely to require hospital admission due to a greater disease severity and a more frequent requirement for intravenous antibiotic treatment, either due to outpatient antibiotic failure or antibiotic resistance. ${ }^{120}$ Patients with $P$. aeruginosa colonisation also have worse health related quality of life using the St. Georges Respiratory Questionnaire, more frequent exacerbations, and worse lung function in cross-sectional studies. $120265271-273$

The relationship between $P$. aeruginosa and lung function decline has been evaluated in relatively few patients and shows inconsistent results. Two studies show no relationship between $P$. aeruginosa and $\mathrm{FEV}_{1}$ decline, ${ }^{15} 274$ while two show a significant relationship. ${ }^{271} 275$ Therefore no firm conclusion on this relationship can be made without further studies.

There is less evidence for a prognostic impact of organisms other than $P$. aeruginosa. One large study shows that colonisation with any organism other than $P$. aeruginosa is associated with a poorer outcome compared with patients not colonised with pathogens. ${ }^{120}$

There is limited data on prognosis with specific pathogens such as NTM and A. fumigatus. ${ }^{267} 268276$ There is a consistent relationship between airway bacterial load and airway inflammation across several studies using both sputum and bronchial wash. 168277278

An increasing number of studies using molecular techniques to characterise the airway microbiome are being published. ${ }^{279} 280$ Microbiome studies demonstrate that loss of bacterial diversity is associated with worse disease defined by $\mathrm{FEV}_{1}$ or exacerbation frequency. ${ }^{279}$ While this has been a valuable research tool, at present this is not available for daily practice and the extent to which it provides clinically relevant information beyond that available from culture has not been established.

\section{Evidence statements}

Chronic colonisation with $P$. aeruginosa is independently associated with

Higher mortality during follow-up $(2++)$

Higher risk of admissions to hospital $(2++)$

Increased exacerbations $(2++)$

Poorer quality of life $(2++)$

Worse radiological severity of disease $(2+)$

Lower FEV ${ }_{1}$ and FVC cross-sectionally $(2++)$

Chronic colonisation with pathogens other than $P$. aeruginosa are associated with

Hospital admissions $(2++)$

Greater airway inflammation $(2++)$

Sputum bacterial load is associated with

Greater airway inflammation $(2++)$

More frequent exacerbations and hospital admissions $(2+)$

\section{Recommendations}

> Consider patients with chronic $P$. aeruginosa colonisation at higher risk of bronchiectasis-related complications. (B)

$>$ Perform regular sputum microbiology screening for patients with clinically significant bronchiectasis to monitor for pathogens and detect new isolation of $P$. aeruginosa. (C) 


\section{SECTION 11}

\section{What is the evidence for the role of viruses/fungal disease in patients with bronchiectasis?}

A single prospective study has reported on the prevalence of respiratory viruses in adult patients with bronchiectasis. Nasopharyngeal swabs and sputum samples, collected from 119 patients at baseline, when clinically stable and at exacerbation, were assayed for 16 respiratory viruses. Viruses were found more frequently in nasopharyngeal swabs and sputum during exacerbations (49/100, $49.0 \%)$ than during steady state $(11 / 58,19 \%)$. Virus-positive exacerbations were associated with a greater increase in markers of systemic and airway inflammation but no differences in lung function, quality of life, and bacterial density were apparent. ${ }^{281}$

Similarly, data regarding the potential role of fungi in bronchiectasis is lacking. A retrospective cohort study was performed using 252 patients from 4 Centres in Spain. ${ }^{282}$ All patients had at least one sputum sample cultured for fungi, with a mean \pm SD of $7 \pm 6$ cultures per patient. Persistent Aspergillus spp. and C. albicans were defined as the presence of 2 or more positive sputum cultures for Aspergillus spp. and C. albicans, respectively, taken at least 6 months apart; eighteen (8.7\%) and 71 (34.5\%) patients had persistent positive cultures for Aspergillus spp. and C. albicans, respectively. Logistic regression, to determine the most significant predictors of fungal persistence, revealed that the presence of mucopurulent or purulent sputum was associated with persistence of Aspergillus spp. with long-term antibiotic therapy associated with persistence of C. albicans.

\section{Evidence statement}

There is insufficient evidence to evaluate the role of fungi and viruses in patients with bronchiectasis. (2-)

\section{Good practice points}

$\checkmark$ Testing to detect viral infection should be considered in patients with an exacerbation of bronchiectasis.

$\checkmark$ Do not routinely use anti-fungal therapy without evidence of fungal disease. Fungal cultures can be positive on those receiving long-term antibiotic therapy.

\section{SECTION 12}

\section{Does eradication of potentially pathogenic microorganisms} improve outcomes in patients with stable bronchiectasis?

There is one randomised controlled study that directly addresses whether antibiotic treatment following first isolation of $P$. aeruginosa improves outcomes in patients with bronchiectasis. Six RCTs report the frequency of negative cultures following antibiotic treatment and one retrospective study reports the outcomes of antibiotic regimens prescribed specifically for $P$. aeruginosa eradication.

In a randomised, but unregistered, study evaluating the effect of 2 weeks of ceftazidime and tobramycin followed by either nebulised tobramycin $300 \mathrm{mg}$ (Tobi) or placebo twice daily for 3 months in 35 patients with bronchiectasis and a first growth of $P$. aeruginosa, the median time to recurrence of $P$. aeruginosa was higher $(\mathrm{P}=0.048)$ in the nebulised tobramycin group $(\mathrm{n}=11)$ compared with placebo group $(\mathrm{n}=17)$, with the proportion free of $P$. aeruginosa after 12 months follow-up being $55 \%$ and $29 \%$, respectively. ${ }^{283}$ The number of exacerbations $(1.27 \pm 1.62$ vs $2.5 \pm 1.63, \mathrm{P}=0.04)$, the number of hospital admissions $(0.06 \pm 0.25$ vs $0.47 \pm 0.51, \mathrm{P}=0.04)$ and the number of days in hospital $(0.90 \pm 3.01$ vs $13.56 \pm 22.64, \mathrm{P}=0.03)$ were also significantly lower in the nebulised tobramycin group. Five patients in the nebulised tobramycin group discontinued treatment due to bronchospasm and two patients in the placebo group withdrew from the study.

In a randomised single blind trial evaluating the effect of nebulised gentamicin in patients with bronchiectasis, negative sputum cultures after 12 months treatment were more common in the gentamicin group $(18 / 27,67 \%)$ compared with the control group $(1 / 30,3 \%) .^{201}$ However, a lesser treatment effect was seen in patients with $P$. aeruginosa at baseline, with 7/13 (54\%) gentamicin treated patients and 9/12 (75\%) control patients reculturing the organism after 12 months treatment. In the whole group, gentamicin treatment was also associated with less sputum purulence (9\% vs 39\%; $\mathrm{P}<0.0001)$; greater exercise capacity (510 (350-690) $\mathrm{m}$ vs. $415(268-530) \mathrm{m} ; \mathrm{P}=0.03)$; and fewer exacerbations $(0$ $(0-1)$ vs. $1.5(1-2) ; \mathrm{P}<0.0001)$ with increased time to first exacerbation (120 [87-162] days vs. 62 [21-123] days; $\mathrm{P}=0.02)$. The gentamicin group had greater improvements in Leicester Cough Questionnaire $(81 \%$ vs $20 \%$; $\mathrm{P}<0.01)$ and St. George's Respiratory Questionnaire $(88 \%$ vs $19 \% ; \mathrm{P}<0.004)$ score. However, 3 months after treatment cessation, all outcome measures were similar to baseline.

In a randomised double blind placebo controlled trial evaluating the effect of dual release liposomal ciprofloxacin in patients with bronchiectasis and ciprofloxacin sensitive $P$. aeruginosa, failure to culture $P$. aeruginosa after 28 days treatment was more common in the ciprofloxacin group compared with the control group $(12(60 \%)$ vs $3(14 \%)$, OR $9.5,95 \%$ CI 1.8 to $63.0, \mathrm{P}=0.003) .{ }^{199}$ In the per protocol analysis, time to first exacerbation was delayed in the ciprofloxacin group compared with the placebo group (134 vs 58 days, $\mathrm{P}=0.046$ ).

In a randomised double blind placebo controlled trial evaluating the effect of dry powder ciprofloxacin in patients with bronchiectasis and predefined potential respiratory pathogens including $P$. aeruginosa and $H$. influenzae, negative bacterial cultures after 28 days treatment was more common in the ciprofloxacin group compared with the placebo group (14 (35\%) vs $4(8 \%), \mathrm{P}=0.001){ }^{198}$

In a 12 month randomised double blind placebo controlled trial evaluating the effect of erythromycin in patients with bronchiectasis, eradication of pathogens (defined as a negative end of treatment sputum culture in participants with pathogenic bacteria identified in baseline samples) occurred more commonly in the erythromycin group compared with the placebo group $(17$ (30\%) vs $6(11 \%)$, OR $3.6,95 \%$ CI 1.3 to $10.6, \mathrm{P}=0.01) .{ }^{192}$ Overall, erythromycin treated patients also had fewer protocol defined pulmonary exacerbations, a lower 24 hours sputum volume and a smaller lung function decline over 12 months compared with controls. However, two contemporary RCTs evaluating the effect of azithromycin in patients with bronchiectasis found no difference between azithromycin and placebo treated patients regarding the eradication of respiratory pathogens (despite there being significant clinical benefits associated with azithromycin treatment) ${ }^{190} 191$ and two subsequent meta-analyses of macrolide trials in this patient population found no overall benefit in this respect. ${ }^{193} 196$

A retrospective study assessed the clinical and microbiological outcomes of eradication therapy following initial $P$. aeruginosa isolation. ${ }^{284}$ Eradication therapy involved intravenous antibiotics $(n=12)$, intravenous antibiotics followed by oral ciprofloxacin $(n=13)$, or ciprofloxacin alone $(n=5)$, combined with 3 months of nebulised colistin. P. aeruginosa was eradicated from sputum in 24 patients (80.0\%). While 11/24 (46\%) subsequently recultured P. aeruginosa (median time 6.2 months), isolates were not genotyped to assess whether this represented relapse or reinfection. Exacerbation frequency was significantly reduced from 3.9 per 
year pre-eradication to 2.1 post-eradication $(\mathrm{P}=0.002)$. Admission rates were unchanged. $20 / 30$ patients reported initial clinical improvement, while at 1 year follow-up, 19/21 had further improved or remained stable. Lung function was unchanged.

Patients with bronchiectasis may also culture a number of other drug resistant pathogens such as MRSA, Stenotrophomonas maltophilia and Achromobacter species. ${ }^{280}$ While it is not known if antibiotics prescribed following first isolation reliably result in eradication or improved patient outcomes, in clinical practice antibiotics are often prescribed in an attempt to prevent chronic infection.

A recent meta-analysis of eight randomised controlled trials recruiting 539 patients showed that long-term inhaled antibiotics reduced sputum bacterial density and augmented eradication of sputum $P$. aeruginosa. No evidence showed higher risk of $P$. aeruginosa resistance after inhaled therapy. In addition, nebulized therapy reduced the number of patients with exacerbation. However, patients with inhaled antibiotics were more likely to suffer wheeze and bronchospasm. ${ }^{207}$

In summary, the available RCT data suggest that inhaled antibiotics improve outcomes in patients with bronchiectasis and that a proportion of patients' cultures convert as a result of treatment. However, only one RCT evaluated outcomes of treatment prescribed to eradicate the first growth of a microorganism. The most clinically relevant definition of eradication has not been established (single negative culture vs sequential negative cultures vs negative PCRs); and it is not known if true bacterial eradication is an achievable endpoint in patients with bronchiectasis, nor whether it is superior to reducing bacterial density at improving patient outcomes. Apparent clearance of $P$. aeruginosa is achieved in some studies. However, the most cost effective antibiotic regimen has not been established, nor whether relapse occurs due to re-emergence of the original strain or de novo infection. Furthermore, it appears that spontaneous $P$. aeruginosa clearance can occur, as some patients only culture $P$. aeruginosa intermittently. ${ }^{266} 274$

\section{Evidence statements}

In patients with bronchiectasis and a first growth of $P$. aeruginosa, treatment with 2 weeks of intravenous ceftazidime +tobramycin followed by 3 months of nebulised tobramycin (Tobi) results in a higher median time to recurrence of $P$. aeruginosa, a reduced exacerbation frequency and a reduced admission rate/days in hospital compared with treatment with 2 weeks of intravenous antibiotics followed by 3 months of nebulised $0.9 \%$ saline. (1-)

\section{Recommendations}

> Offer patients with bronchiectasis associated with clinical deterioration and a new growth of $P$. aeruginosa (1st isolation or regrowth in the context of intermittently positive cultures) eradication antibiotic treatment: (first line treatment: ciprofloxacin 500-750 mg bd for 2 weeks; second line treatment: iv anti-pseudomonal beta-lactam \pm aniv aminoglycoside for 2 weeks, followed by a 3 month course of nebulised colistin, gentamicin or tobramycin). (D)

$>$ Discuss with patients the potential risks and benefits of starting eradication antibiotic treatment versus clinical observation following a new growth of $P$. aeruginosa in the context of stable bronchiectasis. This will include consideration of the likelihood of achieving sustained eradication, the risk of developing chronic infection, and the risk of adverse events with each management approach. (D)

> Offer patients with bronchiectasis associated with clinical deterioration and a new growth of methicillin-resistant S. aureus (MRSA) (1st isolation or regrowth in the context of intermittently positive cultures) eradication. This should be attempted especially in view of infection control issues. (D)

\section{Good practice point}

$\checkmark$ Send sputum for culture and sensitivity immediately before and at each clinical attendance following antibiotics prescribed for bacterial eradication to determine the outcome of treatment.

\section{Research recommendation}

A randomised control trial of $P$. aeruginosa eradication therapy is needed to determine the microbiological and clinical outcomes of eradication therapy.

\section{SECTION 13}

\section{Exacerbations}

Does antibiotic therapy improve outcomes in patients with an exacerbation of bronchiectasis?

Antibiotics are used to treat exacerbations that present with an acute deterioration (usually over several days) with worsening local symptoms (cough, increased sputum volume or change of viscosity, increased sputum purulence with or without increasing wheeze, breathlessness, haemoptysis) and/or systemic upset.

There are no randomised placebo-controlled studies evaluating the efficacy of antibiotics in exacerbations in adults. An RCT performed by Bilton et $a l^{285}$ compared oral ciprofloxacin (in treatment doses) plus placebo to oral ciprofloxacin plus inhaled tobramycin. The addition of inhaled tobramycin led to improved microbiological outcome but the inability to demonstrate an additional clinical benefit may have been due to emergent wheeze resulting from treatment. A case control study by Chalmers et al demonstrated antibiotics led to a reduction in airway inflammatory markers but no clinical endpoints were reported and there was no follow-up beyond 2 weeks. ${ }^{168}$

Cohort studies by Murray et $a l^{286}$ and Bedi et $a l^{287}$ showed that in patients that needed intravenous antibiotic therapy according to the BTS guidelines 2010, they had a good clinical response. There was, however, no control group that did not receive antibiotic therapy.

\section{Evidence statement}

There is insufficient evidence to evaluate the efficacy of antibiotics in exacerbations in adults with bronchiectasis. (2-)

\section{Good practice points}

$\checkmark$ A patient self management plan should be considered, an example is provided here https://www.britthoracic.org.uk/standards-of-care/quality-standards/ bts-bronchiectasis-quality-standards/)

$\checkmark$ There should be prompt treatment of exacerbations and suitable patients should have antibiotics to keep at home.

$\checkmark$ Previous sputum bacteriology results can be useful in deciding which antibiotic to use. Table 6 highlights the firstline and alternative treatments for the common bacterial pathogens implicated in exacerbations of bronchiectasis.

$\checkmark$ Where possible, sputum (spontaneous or induced) should be obtained for culture and sensitivity testing prior to commencing antibiotics.

$\checkmark$ Empirical antibiotics can then be started while awaiting sputum microbiology.

$\checkmark$ Once a pathogen is isolated, antibiotics can be modified if there is no clinical improvement, with treatment guided by antibiotic sensitivity results. 
Table 6 Common organisms associated with acute exacerbation of bronchiectasis and suggested antimicrobial agents- adults

\begin{tabular}{|c|c|c|c|c|}
\hline Organism & Recommended first line treatment & $\begin{array}{l}\text { Length of } \\
\text { treatment }\end{array}$ & Recommended second line treatment & $\begin{array}{l}\text { Length } \\
\text { of treatment }\end{array}$ \\
\hline Streptococcus pneumoniae & $\begin{array}{l}\text { Amoxicillin } \\
500 \mathrm{mg} \text { Three times a day }\end{array}$ & 14 days & Doxycycline $100 \mathrm{mg} \mathrm{BD}$ & 14 days \\
\hline $\begin{array}{l}\text { Haemophilus influenzae- beta lactamase } \\
\text { negative }\end{array}$ & $\begin{array}{l}\text { Amoxicillin } \\
500 \text { mg Three times a day } \\
\text { Or } \\
\text { Amoxicillin } \\
1 \mathrm{G} \text { Three times a day } \\
\text { Or } \\
\text { Amoxicillin } 3 \mathrm{G} \mathrm{BD}\end{array}$ & 14 days & $\begin{array}{l}\text { Doxycycline } 100 \mathrm{mg} \mathrm{BD} \\
\text { Or } \\
\text { Ciprofloxacin } \\
500 \mathrm{mg} \text { or } 750 \mathrm{mg} \mathrm{BD} \\
\text { Or } \\
\text { Ceftriaxone } 2 \mathrm{G} \mathrm{OD} \mathrm{(IV)}\end{array}$ & 14 days \\
\hline $\begin{array}{l}\text { Haemophilus influenzae- beta lactamase } \\
\text { positive }\end{array}$ & $\begin{array}{l}\text { Amoxicillin with clavulanic acid } 625 \\
\text { one tablet Three times a day }\end{array}$ & 14 days & $\begin{array}{l}\text { Doxycycline } \\
100 \mathrm{mg} \text { bd } \\
\text { Or } \\
\text { Ciprofloxacin } \\
500 \mathrm{mg} \text { or } 750 \mathrm{mg} \mathrm{BD} \\
\text { Or } \\
\text { Ceftriaxone 2G OD (IV) }\end{array}$ & 14 days \\
\hline Moraxella catarrhalis & $\begin{array}{l}\text { Amoxicillin with clavulanic acid } 625 \\
\text { one tablet Three times a day }\end{array}$ & 14 days & $\begin{array}{l}\text { Clarithromycin } 500 \mathrm{mg} \mathrm{BD} \\
\text { Or } \\
\text { Doxycycline } 100 \mathrm{mg} \mathrm{BD} \\
\text { Or } \\
\text { Ciprofloxacin } \\
500 \mathrm{mg} \text { or } 750 \mathrm{mg} \mathrm{BD}\end{array}$ & 14 days \\
\hline Staphylococcus aureus (MSSA) & $\begin{array}{l}\text { Flucloxacillin } \\
500 \mathrm{mg} \text { Four times a day }\end{array}$ & 14 days & $\begin{array}{l}\text { Clarithromycin } \\
500 \text { mg BD } \\
\text { Or } \\
\text { Doxycycline } 100 \mathrm{mg} \mathrm{BD} \\
\text { Or } \\
\text { Amoxicillin with clavulanic acid } 625 \text { one tablet Three } \\
\text { times a day }\end{array}$ & 14 days \\
\hline $\begin{array}{l}\text { Staphylococcus aureus (MRSA) } \\
\text { Oral preparations }\end{array}$ & $\begin{array}{l}\text { Doxycycline } 100 \mathrm{mg} \mathrm{BD} \\
\text { Rifampicin }(<50 \mathrm{Kg}) \\
450 \mathrm{mg} \mathrm{OD} \\
\text { Rifampicin }(>50 \mathrm{Kg}) \\
600 \mathrm{mg} \mathrm{OD} \\
\text { Trimethoprim } \\
200 \mathrm{mg} \mathrm{BD}\end{array}$ & 14 days & $\begin{array}{l}\text { Third line } \\
\text { Linezolid } \\
600 \mathrm{mg} \mathrm{BD}\end{array}$ & 14 days \\
\hline $\begin{array}{l}\text { Staphylococcus aureus (MRSA) } \\
\text { Intravenous preparations }\end{array}$ & $\begin{array}{l}\text { Vancomycin } 1 \mathrm{gm} \mathrm{BD*} \text { (monitor serum } \\
\text { levels and adjust dose accordingly) or } \\
\text { Teicoplanin } 400 \mathrm{mg} \mathrm{OD}\end{array}$ & 14 days & $\begin{array}{l}\text { Linezolid } \\
600 \mathrm{mg} \mathrm{BD}\end{array}$ & 14 days \\
\hline Coliforms for example, Klebsiella, enterobacter & $\begin{array}{l}\text { Oral Ciprofloxacin } \\
500 \mathrm{mg} \text { or } 750 \mathrm{mg} \text { BD }\end{array}$ & 14 days & $\begin{array}{l}\text { Intravenous } \\
\text { Ceftriaxone } \\
2 \mathrm{G} \mathrm{OD}\end{array}$ & 14 days \\
\hline Pseudomonas aeruginosa & $\begin{array}{l}\text { Oral Ciprofloxacin } \\
500 \mathrm{mg} \text { bd } \\
\text { ( } 750 \mathrm{mg} \text { bd in more severe infections) }\end{array}$ & 14 days & $\begin{array}{l}\text { Monotherapy: } \\
\text { Intravenous } \\
\text { Ceftazidime } \\
2 \mathrm{G} \text { TDS } \\
\text { or } \\
\text { Piperacillin with tazobactam } \\
4.5 \mathrm{G} \text { TDS } \\
\text { or } \\
\text { Aztreonam } \\
2 \mathrm{G} \text { TDS } \\
\text { or } \\
\text { Meropenem } \\
2 \mathrm{G} \text { TDS } \\
\text { Combination therapy } \\
\text { The above can be combined with gentamicin or } \\
\text { tobramycin or } \\
\text { Colistin } 2 \mathrm{MU} \text { TDS (under } 60 \text { kg, } 50 \text { 000-75 } 000 \text { Units/kg } \\
\text { daily in } 3 \text { divided doses) } \\
\text { Patients can have an in vivo response despite in vitro } \\
\text { resistance. Caution with aminoglycosides as highlighted } \\
\text { below but also if previous adverse events, particularly } \\
\text { previous } \\
\text { ototoxicity/acute kidney injury due to aminoglycosides }\end{array}$ & 14 days \\
\hline
\end{tabular}

BNF 72 (March 2017); OD once daily; BD, twice daily; IV intravenous.

Caution with aminoglycosides in pregnancy, renal failure, elderly or on multiple other drugs.

*Elderly (over 65 years), $500 \mathrm{mg}$ Vancomycin every 12 hours or $1 \mathrm{~g}$ once daily. 
$\checkmark \quad$ In general, antibiotic courses for 14 days are standard and should always be used in patients infected with $P$. aeruginosa. Shorter courses may suffice in patients with mild bronchiectasis.

$\checkmark$ Intravenous antibiotics should be considered when patients are particularly unwell, have resistant organisms or have failed to respond to oral therapy (this is most likely to apply to patients with $P$. aeruginosa).

\section{SECTION 14 \\ Specific treatments \\ What treatments improve outcomes in patients with bronchiectasis and Allergic Broncho-Pulmonary Aspergillosis (ABPA)?}

Limited evidence suggests that if clinical and immunological features of ABPA are identified before radiological features are present, without evidence of proximal bronchiectasis, progressive lung damage occurs rarely once treatment is started. ${ }^{288}$

There are no studies to identify how many patients with bronchiectasis are sensitised to A. fumigatus or have seropositive ABPA.

The aims of treatment of ABPA are to control symptoms, prevent and treat exacerbations, reduce inflammation and prevent further progression and damage to the lung. ${ }^{289}$

The mainstay of treatment is immunosuppression with corticosteroids, with or without the addition of antifungal agents.

\section{Oral corticosteroids}

There are no randomised controlled trials of corticosteroid use in patients with ABPA and bronchiectasis.

Corticosteroids are the mainstay of therapy. Starting and maintenance doses vary in the literature.

A tapering dose of corticosteroid is usually used with monitoring of total serum IgE every 6-8 weeks as a marker of disease activity. Regimes vary from Prednisolone, $0.5 \mathrm{mg} / \mathrm{kg} / \mathrm{d}$, for $1-2$ weeks, then on alternate days for $6-8$ weeks before tapering by $5-10 \mathrm{mg}$ every 2 weeks and discontinue ${ }^{290}$ to more prolonged courses of Prednisolone, $0.75 \mathrm{mg} / \mathrm{kg}$, for 6 weeks, $0.5 \mathrm{mg} / \mathrm{kg}$ for 6 weeks, then tapered by $5 \mathrm{mg}$ every 6 weeks to continue for a total duration of at least 6 to 12 months. ${ }^{81291}$

Patients are considered in remission when they remain without pulmonary infiltrates and/or eosinophilia for 6 months after oral steroid withdrawal. ${ }^{290}$

\section{Inhaled corticosteroids}

Inhaled corticosteroids (ICS) provide a theoretical advantage to oral corticosteroids (OCS) by minimising systemic effects.

The role of high dose ICS was evaluated in a retrospective study of patients with seropositive ABPA and asthma. The conclusion was that there was no role for ICS in isolation as a treatment although they could provide a useful adjunct alongside oral steroids to better control asthma symptoms. ${ }^{292}$

Other small case studies had suggested a role for ICS in weaning patients from high doses of OCS who were otherwise difficult to wean with reported relapses in disease activity when doses of OCS were reduced. ${ }^{293}$

There is no data to support or refute the use of ICS in patients with ABPA and bronchiectasis.

\section{Intravenous corticosteroids}

There are very few studies evaluating the role of pulsed IV steroid therapy in ABPA and none in bronchiectasis with ABPA.
A study of pulsed therapy with monthly intravenous methylprednisolone, in conjunction with itraconazole, in nine patients with CF and ABPA was shown to be effective with all patients showing clinical and laboratory improvement - $\mathrm{FEV}_{1}$ increase, serum IgE levels and total eosinophil counts. ${ }^{294}$ There is no evidence to support the use of IV steroid in patients with ABPA and bronchiectasis.

\section{Azoles}

Itraconazole has been shown in a randomised controlled trial to reduce the need for corticosteroids with fewer exacerbations requiring oral corticosteroids in those treated with itraconazole than in the placebo group. ${ }^{295}$

Other studies of patients with asthma and ABPA have suggested a reduction or elimination of glucocorticoid use while on itraconazole. ${ }^{296}$

A Cochrane review of the role of azoles in ABPA associated with asthma concluded that itraconazole modifies the immunologic activation associated with ABPA and improves clinical outcome, at least over a period of 16 weeks, but noted that adrenal suppression with inhaled corticosteroids and itraconazole is a potential concern. ${ }^{297}$

In current UK practice, itraconazole is used for patients who are steroid dependent and for those who have relapsed after a course of steroids. On occasion it has been utilised from the outset in conjunction with commencement of steroid therapy. The recommended adult dose for itraconazole is $100-200 \mathrm{mg}$ twice daily. ${ }^{289}$

A systematic review of the efficacy and safety of antifungal treatments in patients with ABPA and either asthma or cystic fibrosis, including itraconazole, voriconazole, posaconazole, ketoconazole, natamycin, nystatin and amphotericin B, concluded that there was an improvement in symptoms, frequency of exacerbations and lung function in most of the studies and this was seen more commonly with the azoles. ${ }^{298}$

There is however a lack of controlled studies evaluating the use of antifungal agents.

Voriconazole and posaconazole are reported to be an effective adjunct therapy in the management of ABPA, with clinical improvement in $70 \%$ of patients with ABPA treated with voriconazole and $78 \%$ of patients with ABPA treated with posaconazole in studies concerning patients with severe asthma. ${ }^{299}$

There are no studies concerning the use of these antifungals in patients with bronchiectasis.

\section{Amphotericin B and anti-lgE therapies}

The potential role of agents such as amphotericin B and monoclonal antibody therapy with agents such as omalizumab, are of interest but require further evaluation in ABPA.

\section{Monitoring response to treatment}

Monitoring response to treatment through repeat measurement of Aspergillus serology, eosinophils and repeat chest radiology can aid weaning of treatment. A study of 81 patients with bronchiectasis and ABPA suggested that the reduction in total $\mathrm{IgE}$ was most sensitive to change and most reflective of successful treatment. $^{79}$

\section{Evidence statements}

Itraconazole use in corticosteroid dependent patients is associated with a reduction in corticosteroid dose, improved pulmonary function and improved exercise capacity. $(1+)$ 
Total $\mathrm{IgE}$ is the most sensitive marker for monitoring treatment response. $(2+)$

\section{Recommendations}

$>$ Offer oral corticosteroid to patients with active ABPA. An initial dose of $0.5 \mathrm{mg} / \mathrm{kg} / \mathrm{d}$ for 2 weeks is recommended. Wean steroids according clinical response and serum $\operatorname{IgE}$ levels. (D)

> Consider itraconazole as a steroid sparing agent for patients dependent on oral corticosteroids where difficulty in weaning is experienced. (B)

> Monitor patients with active ABPA with total IgE level to assess treatment response. $(\mathrm{C})$

\section{Does immunoglobulin replacement treatment therapy improve outcomes in patients with bronchiectasis due to antibody deficiency?}

There are no double-blind randomised placebo-controlled trials of immunoglobulin replacement in patients with either primary or secondary antibody deficiency. Cohort studies show that introduction of $\operatorname{IgG}$ replacement therapy is associated with a reduced incidence of pneumonia in patients with CVID and XLA. ${ }^{300-303}$

Randomised controlled trials comparing high dose and low dose intravenous immunoglobulin replacement therapy show that higher doses are associated with reduced incidence of acute sino-pulmonary ${ }^{304}$ and upper respiratory tract infections. ${ }^{305}$ Meta-analysis and cohort studies show an inverse relationship between trough IgG levels and rate of serious infections/and or pneumonia in patients with CVID and XLA receiving intravenous IgG therapy. ${ }^{300}$ 306-308

Current guidelines suggest that patients with CVID and bronchiectasis should be treated with an IgG replacement dose of $0.6 \mathrm{~g} / \mathrm{Kg} / \mathrm{month}$ compared with standard treatment of $0.4 \mathrm{~g} /$ $\mathrm{Kg} / \mathrm{month}^{96}$ There is however no consensus on the dose of IgG replacement to treat lung disease in patients with primary antibody deficiency or the optimal trough IgG levels to prevent pneumonia and chest infections. North American guidelines for the use of $\operatorname{IgG}$ therapy in primary immune deficiency recommend that the goal of therapy should be to improve clinical outcomes rather than aim for a specific trough concentration. 309310

There is no conclusive data to suggest that IgG replacement therapy prevents the progression of bronchiectasis and other pulmonary complication of primary antibody deficiency syndrome. Two longitudinal studies of patients with CVID and XLA on standard IgG replacement doses over 7-8 years showed greater than anticipated annual declines in airflow obstruction and transfer factor. ${ }^{311} 312$ Two European studies showed that the prevalence of bronchiectasis on CT scan increased over time in patients with primary antibody syndrome on IgG replacement therapy. ${ }^{300313}$ The prevalence of bronchiectasis increased from $47.3 \%$ to $54.7 \%$ in CVID patients and from $33 \%$ to $39 \%$ in XLA patients over 5 years. ${ }^{300}$

\section{Evidence statements}

Cohort studies and randomised trials of high versus low-dose immunoglobulin replacement therapy in patients with CVID and XLA show a significant reduction in the rate of serious infections including pneumonia. $(2++)$

IgG replacement therapy may be useful in patients with minor antibody deficiency syndromes (specific polysaccharide antibody deficiency and/or (IgA or IgG subclass) deficiencies with absent/ impaired pneumococcal antibodies vaccine responses) who continue to have objective evidence of bacterial sino-pulmonary infection and progressive disease despite appropriate management of bronchiectasis by a respiratory physician and physiotherapist. $(2+)$

There is no definite data to suggest that $\operatorname{IgG}$ replacement therapy prevents onset of bronchiectasis, or delays progression in bronchiectasis. $(2+)$

\section{Recommendations}

> Offer IgG therapy to all patients with CVID and XLA. (B)

$>$ Consider IgG therapy for patients with specific polysaccharide antibody deficiency and/or IgA deficiency or IgG subclass deficiencies with absent/impaired pneumococcal vaccine antibody responses who continue to have objective evidence of bacterial sino-pulmonary infection and progressive disease despite appropriate management of bronchiectasis. (C)

\section{Good practice points}

$\checkmark$ All patients receiving IgG replacement therapy should be under the joint care of a clinical immunologist and respiratory specialist in bronchiectasis.

$\checkmark$ Offer patients receiving replacement IgG the choice of hospital or home-based therapy.

\section{Research recommendation}

Randomised controlled trials are needed to assess which patients with bronchiectasis would benefit from long term Immunoglobulin $\mathrm{G}$ replacement therapy alone or as an adjunct to long term antibiotic therapy- assessing the optimal dose of $\operatorname{IgG}$ replacement and identification of ideal trough IgG level to prevent recurrent infections.

Gastro-oesophageal reflux disease (GORD) and Bronchiectasis There is an association between gastro-oesophageal reflux disease (GORD) and bronchiectasis. An increased prevalence of proximal and distal GORD (40\%) was demonstrated by oesophageal monitoring in a cohort of 27 patients with bronchiectasis compared with controls. This finding was not confined to those with severe lung disease, and the detection of pepsin within sputum in this study did not correlate with the finding of GORD. ${ }^{314}$

Increased severity of bronchiectasis has been observed in patients with significant reflux symptoms. In a cohort study of 163 patients with bronchiectasis, those with reflux symptoms according to the Hull Airways Reflux Questionnaire had a significantly lower $\mathrm{FEV}_{1}$ and a greater number of lobes affected by bronchiectasis on CT scan. There was higher inflammation in sputum and increased chronic colonisation with PPMs. Symptoms of reflux were independently associated with cough severity and exacerbation frequency. ${ }^{98}$ In another study of 100 patients with bronchiectasis, those patients with upper gastrointestinal symptoms also had a greater number of lobes affected and significantly worse lung function. ${ }^{315}$

A possible relationship between $H$. pylori and bronchiectasis symptoms has been described. ${ }^{314}$ Some studies of $H$. pylori serology have shown higher levels in bronchiectasis patients compared with control populations. In one study of 100 patients with bronchiectasis measuring specific IgG to H. pylori, seroprevalence was $76 \%$ compared with $54.3 \%$ of controls. Further analysis showed that those bronchiectasis 
patients who were defined as sputum producers had significantly higher $H$. pylori seroprevalence $(83.1 \%)$ than those defined as non-producers (58.6\%). Multiple regression analysis showed an association between H. pylori IgG concentrations (continuous values) and sputum volume and age. Lung function however did not correlate with $H$. pylori serology in this regression model. ${ }^{316} \mathrm{~A}$ further cohort study of 40 patients with bronchiectasis showed H.pylori seropositivity in 75\% of patients with bronchiectasis in comparison to $51.5 \%$ of healthy control subjects, but there were no significant differences in gastrointestinal symptoms between patients and controls. ${ }^{317}$ On the other hand Angrill et al failed to find differences in $H$. pylori seropositivity between bronchiectasis patients and the general population, and studies of bronchial mucosal specimens taken from patients with bronchiectasis did not demonstrate the presence of H. pylori. ${ }^{318}$

\section{Evidence statement}

GORD is common in patients with bronchiectasis and is associated with increased severity of bronchiectasis suggesting a causal relationship (level 2-).

There is a possible association between $H$. pylori seropositivity and bronchiectasis (2-)

\section{Recommendation}

> Treat GORD symptoms in patients with bronchiectasis according to existing NICE guidance ${ }^{319}$. (D)

\section{Good practice points}

$\checkmark$ GORD should be considered in patients with hiatus hernia, persistent coliforms in sputum samples or recurrent exacerbations with no other cause identified.

$\checkmark \quad$ Investigate patients who may have gastro-oesophageal reflux according to local policies.

$\checkmark$ Consider the addition of prokinetic agents if symptoms of GORD increase with an airway clearance technique in sitting position. Avoid eating in the hour immediately prior to physiotherapy.

\section{What is the prevalence of rhinosinusitis in patients with stable bronchiectasis and what are the outcomes of treatment?}

Chronic rhinosinusitis is defined as persistent upper airway symptoms lasting $>12$ weeks and can be subdivided by the presence or absence of nasal polyps. The diagnosis of rhinosinusitis is predominantly clinical and made on history and examination with only a limited role for radiology.

Symptoms of rhinosinusitis are very commonly reported in patients with bronchiectasis. In a UK study, $65 \%$ of bronchiectasis patients had symptoms of rhinosinusitis $(\mathrm{n}=95)$. This was most common in patients with idiopathic disease, affecting $84 \%$ of patients. ${ }^{23}$ In a study of 101 patients with bronchiectasis in Australia, $72 \%$ reported symptoms of rhinosinusitis. ${ }^{15}$

Among patients with upper airway symptoms, Guillemany et al reported that $41.6 \%$ had nasal polyps. ${ }^{320}$ In an unselected bronchiectasis population from Spain ( $\mathrm{n}=91), 80.2 \%$ of stable bronchiectasis patients had rhinosinusitis according to EP30S criteria, and $26.4 \%$ had nasal polyps. ${ }^{321}$ Patients with rhinosinusitis may have worse quality of life and worse radiological bronchiectasis than those without rhinosinusitis. ${ }^{322} 323$

Chronic rhinosinusitis (CRS) can be a feature of idiopathic bronchiectasis, but patients with bronchiectasis and rhinosinusitis should undergo a comprehensive assessment for underlying aetiology as for all patients. Chronic infective rhinosinusitis can be a feature of primary ciliary dyskinesia along with middle ear infections and infertility.

There are no specific randomised controlled trials to guide the management of rhinosinusitis in bronchiectasis. In addition, there is no evidence to demonstrate that treatment of rhinosinusitis will improve respiratory outcomes, such as exacerbation frequency, in bronchiectasis.

In a high proportion of cases, response to treatment for chronic rhinosinusitis is partial and treatments need to be continued over medium to long term. Typical symptoms of nasal polyps include nasal obstruction, hyposmia/anosmia and decreased taste and post-nasal catarrh.

In the absence of specific recommendations and outcome data in bronchiectasis it seems reasonable to follow existing algorithms for the treatment of chronic rhinosinusitis, such as that provided by the European position paper on rhinosinusitis and nasal polyps or the British Society of Allergy and Clinical immunology guidelines. ${ }^{324} 32.5$

Nasal irrigation with $0.9 \%$ saline is safe, inexpensive and widely used and has been shown to reduce symptoms of CRS, including in patients with CRS and PCD. ${ }^{326}$ Topic intranasal steroids are the other first line treatment to suppress inflammation in CRS. ${ }^{326}$ Nasal drops are preferred. Fluticasone and mometasone have the lowest bioavailability and as such as used in preference. ${ }^{327}$ Macrolides have evidence in the management in chronic rhinosinusitis but are not regarded as first line therapy. ${ }^{328}$

A proposed algorithm for initial assessment and treatment is shown in figure 5.

Sinus CT's are rarely helpful and reserved for patients failing medical therapy or those with features suggesting alternative diagnoses such as blood stained discharge, displaced globe or severe pain. ${ }^{326}$

The box above identifies a series of symptoms that indicate an alternative diagnosis, such as orbital symptoms (periorbital oedema, displaced globe, diplopia, ophthalmopegia or reduced visual acuity) severe pain and neurological symptoms. Facial pain without nasal symptoms is rarely due to rhinosinusitis. 2.6

\section{Evidence statements}

Chronic rhinosinusitis is common in bronchiectasis and may contribute to impaired quality of life. $(2+)$

\section{Recommendations}

$>$ The evaluation of patients with bronchiectasis should include assessment of symptoms of chronic rhinosinusitis. (D)

> Patients with bronchiectasis and symptoms of rhinosinusitis should be evaluated and treated according to existing evidence-based treatment pathways. (D)

\section{Should treatment of bronchiectasis be altered in the presence of co-morbidities?}

There are no studies addressing treatment of bronchiectasis in the presence of asthma and atopy although an accumulation of clinical experience suggests that these factors need to be addressed for good control of bronchiectasis to be achieved. ${ }^{329}$ In the presence of this co-morbidity, there is a risk of poor clearance of sputum due to bronchospasm, greater mucus secretion and endobronchial inflammation, all of which is likely to lead to reduction in lung function and poor control of infections. 


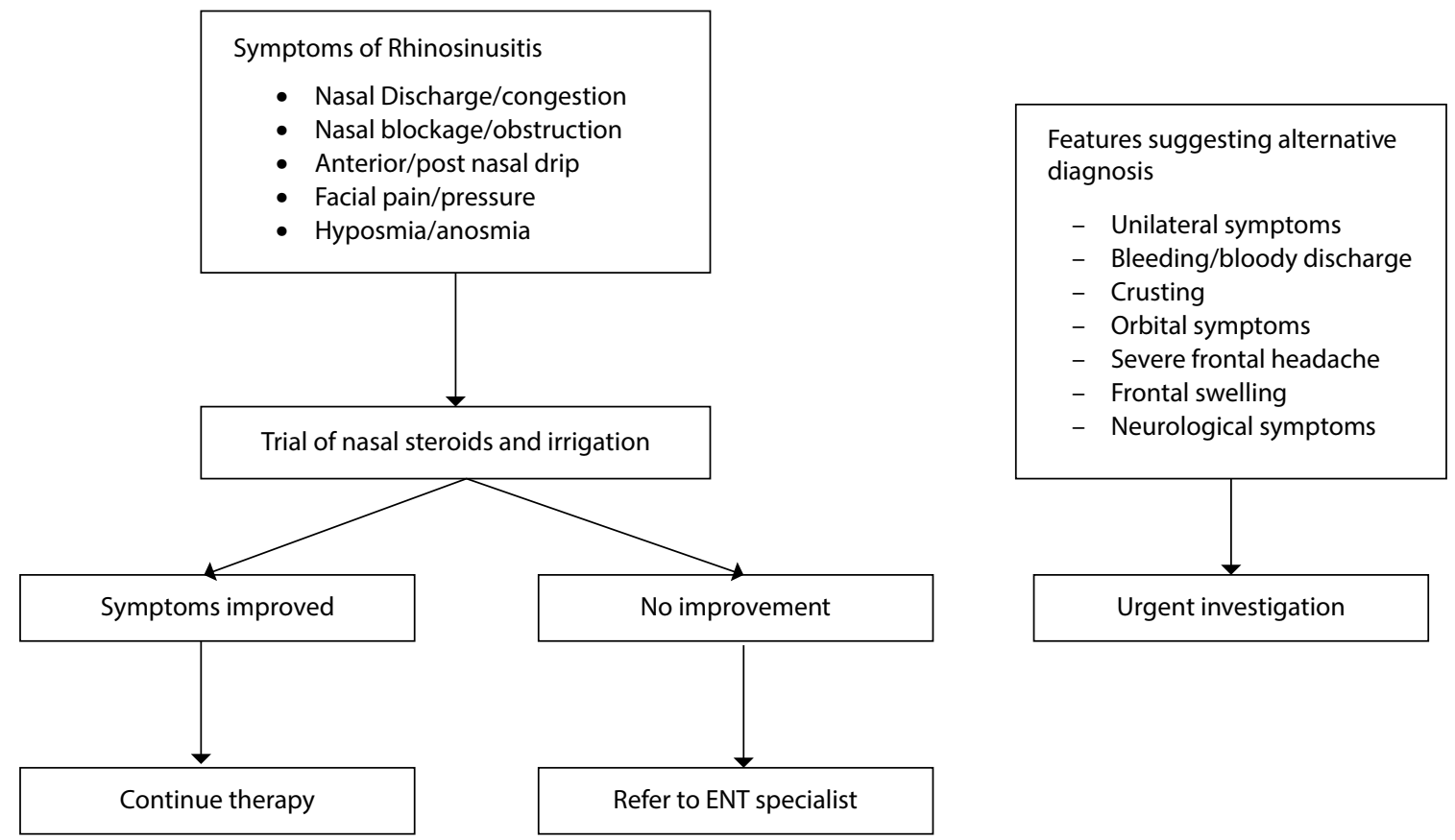

Figure 5 Algorithm for initial assessment and treatment - rhinosinusitis.

Several studies have shown a higher than expected prevalence of bronchiectasis in patients with COPD and these patients probably have a worse prognosis than bronchiectasis without COPD; however there is an acknowledged lack of evidence as to implications for treatment. ${ }^{255} 269330-332$

Patients with HIV-related disease appear to be at greater risk of bronchiectasis than the general population but management implications were not discussed in the studies reviewed. ${ }^{333} 334$

\section{Inflammatory bowel disease}

The majority of studies are undermined by low numbers, which may reflect the relatively small population of patients with bronchiectasis whose condition is associated with inflammatory bowel disease (IBD) (see online appendix $6<1 \%-3 \%)^{23}{ }^{62}$ Kelly et al studied 10 patients (time period not stated) ${ }^{59}$ and Mahadeva et al identified 17 patients over a 14 year period with coexistent IBD and pulmonary disease. ${ }^{60}$ Bronchiectasis appeared to be the most common feature although other abnormalities were seen on CT scan. In some cases a biopsy revealed evidence of lymphocytic inflammation $^{59}$ and a useful clinical response to oral or inhaled corticosteroids was observed. ${ }^{60}$ The nature of the steroid treatment and its outcome were not described in detail although alveolitis and bronchiolitis were reported to resolve fully. ${ }^{60}$

The overlap of IBD and bronchiectasis was comprehensively reviewed in a European Respiratory Monograph in 2011, again with an emphasis on corticosteroid responsiveness although the lack of evidence was acknowledged. ${ }^{335}$

\section{Rheumatoid arthritis}

In a study of the effects of immunosuppression, 37 patients with rheumatic diseases (and nine with IBD) were identified in a database of 539 patients with bronchiectasis: 26 had rheumatoid arthritis (RA). ${ }^{336}$ The majority developed bronchiectasis after the onset of autoimmune disease. All had received either conventional disease modifying anti-rheumatic drugs (DMARDs) mostly methotrexate - or biologics. Methotrexate in particular was associated with a range of adverse effects. Patients with RA or IBD were not found to suffer more exacerbations than patients with bronchiectasis alone, and the frequency of colonising organisms such as $P$. aeruginosa and $H$. influenzae was similar. Longitudinal data were available for a small cohort of patients with RA and bronchiectasis. In these patients, control of RA disease activity appeared to track with spirometry, in that spirometry declined when the DAS score rose, and vice versa. DMARDs did appear to be associated with increased pneumonia and other infections.

The risk of infection in patients using DMARDs or biologics was assessed in a cohort of $>3000$ patients, of whom 86 had both a rheumatological disease and bronchiectasis. ${ }^{337}$ Sufficient data was available on only 47 patients receiving DMARDs or biologics. Of 40 patients with RA and bronchiectasis, 35 were seropositive. 13 had bronchiectasis prior to RA, 27 were diagnosed with bronchiectasis after the onset of RA but it was attributed directly to RA only in 13 cases, otherwise the aetiology was thought to be childhood infection. An OR of 8.7 (95\% CI 1.7 to 43.4 ) was found for respiratory infections in patients treated with biologics compared with traditional DMARDs. The OR for infections was $7.4(2.0-26.8)$ in the presence of previous sputum colonisation by potentially pathogenic micro-organisms. The authors therefore recommended using non-biologics in preference to biologics if possible. Etanercept and rituximab were associated with a lower rate of infections than the other biologics, but the numbers were very small in this study.

\section{Evidence statements}

Patients with bronchiectasis and comorbid COPD have more severe disease and worse prognosis than those with bronchiectasis alone. $(2+)$

Patients with co-morbid rheumatoid arthritis and bronchiectasis may have worse outcome than those with either disease alone. (3)

Immunosuppressive treatment for connective tissue disorders is associated with a higher risk of infection in general; biologics may promote infections more than traditional DMARDs, and the risk may be higher in patients already colonised with potentially pathogenic micro-organisms. (3)

Inflammatory bowel disease-related bronchiectasis may be responsive to corticosteroids. (3) 


\section{Recommendations}

> Consider a trial of inhaled and/or oral corticosteroids in patients with bronchiectasis and IBD. (D)

$>$ Ensure optimal control of asthma and allergies in patients with both bronchiectasis and asthma (D).

> Monitor patients with co-morbid COPD and bronchiectasis as they are at higher risk of death. (D)

- Patients with bronchiectasis and autoimmune conditions should be carefully assessed for autoimmune related lung disease and often require long term follow-up in a secondary care setting. (D)

> Patients with bronchiectasis who require DMARDs or biologics for rheumatoid arthritis should be referred to a chest physician for further assessment before treatment is started. (D)

\section{SECTION 15}

\section{How should we monitor bronchiectasis?}

There are no studies in bronchiectasis comparing different monitoring schedules, or relating particular monitoring strategies to clinical outcomes. Factors associated with increased risk of exacerbations, hospitalisations and mortality in bronchiectasis include age, body mass index, past history of exacerbations and or hospitalisations, persistent infection, especially with P. aeruginosa, dyspnoea as measured on the MRC scale, radiological extent of disease and airflow limitation $\left(\mathrm{FEV}_{1}\right)$ (see tables 6 and 7). These components have recently been operationalised into two multi-component scores BSI ${ }^{120}$ and FACED. ${ }^{119}$ Recently a comparative analysis has suggested BSI has greater sensitivity in predicting those at risk of future risk of exacerbations. ${ }^{121}$ In addition, co-morbidities such as COPD and rheumatoid arthritis have also been shown to affect mortality and therefore should be recorded. ${ }^{269} 338$ The role of the bronchiectasis aetiology and comorbidity index (BACI) in routine practice is yet to be determined. ${ }^{338}$

It seems reasonable to propose that routine monitoring should include reassessment of the variables above that have been shown to be relevant in bronchiectasis. In addition patient monitoring should include assessment of symptoms and the impact that these have on health status. This may be best achieved using a validated tool, of which there are many, ${ }^{339}$ including tools specifically for bronchiectasis such as the QOL-B. ${ }^{340}$ Systematic review has demonstrated that these tools may assess components of health status in bronchiectasis not measured using other outcomes. ${ }^{339}$ Routine monitoring of quality of life may not be needed in all cases but should be considered when higher cost interventions are planned. The optimal tool for monitoring treatment effects has however yet to be determined in bronchiectasis. As in other respiratory disease, exercise capacity can be assessed using 6MWD or ISWT and even small changes in these measures may reflect important changes in health. ${ }^{221}$

The BTS Quality Standards for clinically significant bronchiectasis in adults $2012,{ }^{341}$ based on the 2010 BTS guideline for non-CF bronchiectasis, suggested that patients fulfilling the following criteria should continue to receive specialist input in secondary or specialist care. The guideline development group advise using the following criteria:

1. patients with chronic $P$. aeruginosa, non-tuberculous mycobacteria or methicillin-resistant $S$. aureus colonisation (MRSA);

2. deteriorating bronchiectasis with declining lung function;

3. recurrent exacerbations ( $\geq 3$ per year);

4. patients receiving long term antibiotic therapy (oral, inhaled or nebulised);

5. patients with bronchiectasis and associated rheumatoid arthritis, immune deficiency, inflammatory bowel disease and primary ciliary dyskinesia;

6. patients with allergic bronchopulmonary aspergillosis;

7. patients with advanced disease and those considering transplantation.

\section{Evidence statement}

Variables exist which affect outcome in bronchiectasis and such variables can be monitored over time. ${ }^{119120}(1+)$

No studies have however measured the impact of such monitoring, or demonstrated that action taken in response to changes in measured variables will affect outcome.

\section{Recommendation}

> All patients with bronchiectasis should undergo routine monitoring in order to identify disease progression, pathogen emergence and modify treatment where necessary. (D)

Table 7 Routine monitoring tests

\begin{tabular}{|c|c|c|}
\hline & Mild-disease severity & Moderate - Severe \\
\hline Severity Index scoring & baseline & baseline \\
\hline BMI (Body Mass Index) & annual & annual \\
\hline Exacerbation History & annual & 6 monthly \\
\hline Sputum Culture & annual & 6 monthly \\
\hline MRC Dyspnoea Score & annual & 6 monthly \\
\hline Spirometry & annual & annual \\
\hline CT (Radiological Extent) & at diagnosis* $¥$ & at diagnosis* $\ddagger$ \\
\hline Sputum mycobacterial culture & baselineł & baselineł \\
\hline Oxygen saturation monitoring (Sp02) & annual & 6 monthly \\
\hline Underlying cause investigations & at diagnosis $¥$ & at diagnosisł \\
\hline Comorbidities assessment & at diagnosisł & at diagnosisł \\
\hline
\end{tabular}

${ }^{*}$ Consider repeat CT scanning in patients with primary immunodeficiency with scan interval of 3-5-years. ${ }^{38}$

tThis may need tailored in light of the local prevalence rates of NTM infections and in some centres may need undertaken on a regular basis. Further cultures at exacerbation may be appropriate.

‡Repeat investigations if a deteriorating patient. 


\section{Good practice points}

$\checkmark$ Tailor the frequency of routine monitoring to the patient's disease severity (see table 7).

$\checkmark$ Assess patients annually, and more frequently in more severe disease.

$\checkmark$ Perform pulse oximetry to screen for patients who may need blood gas analysis to detect respiratory failure.

$\checkmark$ A baseline CXR may provide a useful comparator in the event of clinical deterioration.

\section{SECTION 16}

\section{Is there a role for microbiological sensitivity testing? Evidence summary}

Despite widespread use of sensitivity testing to inform antibiotic treatment in patients with bronchiectasis, there are no published RCTs which determine whether antibiotic treatment guided by antibiotic sensitivity results improve clinical outcomes for patients.

RCTs suggest that acute antibiotic treatment rarely results in the development of resistance. For example, Bilton et al reported that $P$. aeruginosa resistance to tobramycin developed in only $1 / 27$ patients receiving oral ciprofloxacin and tobramycin inhalation solution for 14 days. ${ }^{285}$ Likewise, Barker et al reported that only $4 / 36(11 \%)$ patients receiving tobramycin inhalation solution for 4 weeks who began the study with susceptible $P$. aeruginosa had resistant $P$. aeruginosa at their last visit. ${ }^{342}$

Long term inhaled antibiotic treatment only occasionally results in the development of resistance. For example, Murray et al reported that resistance to gentamicin did not develop in 57 patients receiving either nebulised gentamicin or placebo for 1 year. ${ }^{201}$ Similarly, Drobnic et al reported that in a study assessing the efficacy of 6 month tobramycin inhalation therapy in 30 patients, resistant $P$. aeruginosa was only detected in sputum from two patients during the tobramycin treatment period. ${ }^{343}$ Equally, in an RCT comparing treatment with inhaled colistin ( $n=73$ participants) or placebo ( $n=71$ participants) for up to 6 months in patients with chronic $P$. aeruginosa infection, no colistin-resistant strains of $P$. aeruginosa were identified and there were no significant differences in treatment-emergent pathogens between treatment groups. ${ }^{200}$

There has also been considerable focus on the development of resistance with long-term oral treatment with macrolides. In a meta-analysis assessing the clinical effect of long-term macrolides in bronchiectasis, ${ }^{196}$ two studies included reported on emergence of macrolide resistance: Altenburg et al reported that during treatment, 53 of 60 pathogens $(88 \%)$ tested for sensitivity in 20 patients in the azithromycin group became macrolide resistant compared with 29 of 112 pathogens (26\%) in 22 patients in the placebo group $(\mathrm{P}<0.001$ by t-test $) .{ }^{191}$ Serisier et al reported erythromycin significantly increased the proportion of macrolide-resistant commensal oropharyngeal streptococci $(\mathrm{P}<0.001) .{ }^{192}$ However, a meta-analysis was not possible due to the diversity of parameters. Resistance development following long-term treatment with quinolones has also been assessed; Rayner et al $1994^{344}$ reported that, following continuous treatment with oral ciprofloxacin for at least 90 days, resistance to ciprofloxacin developed in two patients with $P$. aeruginosa infection and this was associated with clinical deterioration. ${ }^{344}$

A laboratory based study has questioned the use of current susceptibility testing methods for the complex populations of bacteria found in chronic lung infection. Gilham et al used the disc diffusion method to determine the minimum inhibitory concentration (MIC) of multiple $P$. aeruginosa morphotypes isolated from sputa from patients with bronchiectasis. ${ }^{345}$ There was considerable variation in inhibition zone size between multiple colonies of the same morphotype with the range of zone sizes crossing the breakpoint between resistance and susceptibility in 11/33 samples analysed. This clearly highlighted that sensitivity testing in bronchiectasis is likely to be poorly reproducible with the result depending on the particular colonies tested.

\section{Evidence statements}

There is no evidence to show that antibiotic treatment guided by sensitivity results improves clinical outcomes for patients. ${ }^{4}$

Acute antibiotic treatment only occasionally results in the development of resistance. (1-)

Long-term macrolide use results in the emergence of macrolide resistance. (1-)

\section{Good practice points}

$\checkmark$ Antibiotic sensitivity testing can be used to determine if resistance develops to either acute or long-term antibiotic treatment.

$\checkmark$ Where possible, treatment should be guided by antibiotic sensitivity results but is often empirical based on previous sputum bacteriology.

$\checkmark \quad$ Some patients with an infective exacerbation may respond to antibiotic treatment despite resistance to that drug in vitro. Antibiotics should only be changed if there is no clinical response.

$\checkmark$ For those on long term antibiotic treatment, there should be repeat sensitivity testing where there is a clinical concern regarding loss of efficacy with therapy.

\section{SECTION 17}

\section{Is there any evidence of cross-infection with pathogenic organisms (conventional bacteria and environmental mycobacteria)?}

While there are numerous studies confirming the transmissibility of certain epidemic strains of $P$. aeruginosa and more recently Mycobacterium abscessus (M. abscessus) in cystic fibrosis (CF) there are few studies assessing the potential for cross-infection in bronchiectasis. ${ }^{346} 347$ The available studies of cross infection in bronchiectasis are mostly single centre design, often focus on $P$. aeruginosa, and have relatively limited longitudinal sampling with often limited description of the clinical setting. ${ }^{348} 349$ This latter aspect may be particularly important if facilities are shared with patients who have CF and carry known CF epidemic strains.

De Soyza et al reported a case series of 40 patients from a single adult bronchiectasis clinic in Newcastle, UK, not practising strict segregation but with reported limited sharing of facilities with CF patients. ${ }^{348}$ The study focused on $P$. aeruginosa. It was a predominantly cross- sectional design although it reported longitudinal follow-up of 10 patients for up to 4 years. Their data suggested no single common 'epidemic strain' was found. They identified however that certain common strains that are found widely within the natural environment for example, Pseudomonas Clone $\mathrm{C}$ can be found in patients attending bronchiectasis clinics. This raises the potential for environmental acquisition in the bronchiectasis population.

Pujana et al similarly used a single centre, short-term study using bacterial fingerprinting using a PCR approach. ${ }^{349}$ In 
this study of 16 patients with bronchiectasis from Bilbao, Spain, 64 P.aeruginosa isolates were examined. Cross-infection or common source acquisition did not appear to have occurred. A study of 125 isolates taken from 31 patients attending two clinics in Spain using pulsed field gel electrophoresis suggested certain strains may be predominant within a clinic. ${ }^{350}$

Two papers which have used the higher resolution of whole genome sequencing (WGS) have raised the possibility of cross infection. A summary of studies to date would support the conclusion that significant cross infection with $P$. aeruginosa has not been identified, but longitudinal studies are needed. ${ }^{351} 352$

One study has suggested potentially similar clones of $\mathrm{Myco-}$ bacterium avium (M. avium) are present in patients with chronic lung disease. ${ }^{353}$ The study included some patients with bronchiectasis though confirming the exact number was not possible. The study aims were primarily to compare the benefits of VNTR fingerprinting over PFGE, the present 'gold standard' to allow a worldwide database. This aim was achieved. The authors noted different patients' isolates had the same VNTR types and these were mostly clonal by PFGE suggesting that clonal groups do exist with M. intracellulare. Despite this evidence, the authors provided no evidence or discussion on the question of transmission of M. avium. There are therefore inadequate data to provide a definitive statement on the risk of M. avium transmission in bronchiectasis. There are no extensive data on M. abscessus infection in bronchiectasis but this is a rapidly changing field.

\section{Evidence statements}

There is no evidence of transmissibility of epidemic $P$. aeruginosa in the available cohort studies. (2-)

There is no evidence of transmissibility of $M$. avium in the available cohort studies. (2-)

\section{Recommendation}

> Individual or cohort segregation based on respiratory tract microbiology results is not routinely required for patients with bronchiectasis. (D)

\section{Good practice points}

$\checkmark$ Good cross infection prevention principles should be applied: seek advice on local policies.

$\checkmark \quad$ The transmissibility of $P$. aeruginosa in cystic fibrosis appears more common. In the case of shared facilities with cystic fibrosis patients the cross infection guidelines for cystic fibrosis should prevail.

\section{Research recommendation}

Large scale robust data that confirm or refute the transmissibility of key pathogens such as $P$. aeruginosa and non-tuberculous mycobacteria are needed.

\section{SECTION 18}

\section{Specialist vs non-specialist setting}

There are no studies available describing differences in outcome for patients with bronchiectasis in specialist compared with non-specialist care settings. The consensus team (expert opinion) suggest there may be benefits to patients by attending a specialist clinic for bronchiectasis as this will facilitate access to appropriate diagnostic and management expertise through a multidisciplinary team (for example Respiratory Nurse Specialists, Pharmacists, Consultants with a special interest in bronchiectasis, Immunology Consultants, Respiratory Radiologists, Dieticians and Psychologists). This may be most relevant to patients with severe or complex disease.

\section{Good practice point}

$\checkmark$ Specialist clinics should be considered in patients requiring hospital follow-up.

\section{SECTION 19}

\section{What are the complications of bronchiectasis?}

Complications of bronchiectasis may have changed over the years. Haemoptysis and thoracic infections have always been important, but finger clubbing and metastatic infection such as cerebral abscesses are now rare. ${ }^{15} 354$

\section{Chronic respiratory failure}

Most patients with bronchiectasis have some degree of lung function impairment, but lung function decline over time was only slightly greater than normal in a follow-up study of patients in Edinburgh. ${ }^{355}$ Lung function was shown to be capable of improvement as well as decline in a UK study focusing on potential impact of $P$. aeruginosa colonisation in patients with bronchiectasis. ${ }^{274}$ An Australian study linked risk of decline with higher volume of daily sputum at baseline. ${ }^{15}$

A recent analysis of patients recorded on the EMBARC European bronchiectasis database revealed that 86 of 1145 (7.5\%) patients were using long term oxygen therapy for chronic respiratory failure. ${ }^{356}$

\section{Haemoptysis}

A series at the Mayo Clinic from 1976 to 1993 identified 3421 patients seen for bronchiectasis, of whom 134 patients underwent pulmonary resection. ${ }^{357}$ Haemoptysis was present in 63 of the lung resection group, and was the indication for surgery in $26(19.4 \%)$. The frequency of haemoptysis in medically managed patients was not given.

One study from Korea has suggested that use of inhalers may increase the risk of haemoptysis ${ }^{358}$.

The degree of haemoptysis can vary widely. Streaks of haemoptysis can be seen with chest infections and sometimes in stable patients with severe bronchiectasis who are treated with anti-platelet therapy or anti-coagulant therapy for comorbid conditions.

The volume of blood defining major haemoptysis is not known, with no internationally agreed definition.

\section{Good practice point}

$\checkmark$ If haemoptysis $10 \mathrm{mls}$ or less over a 24 hour period, treat with an appropriate oral antibiotic. If clinical deterioration, arrange emergency admission to hospital.

$\checkmark$ Management of major haemoptysis should be multidisciplinary with involvement of respiratory physicians, interventional radiology and thoracic surgeons. Empirically treat patients with intravenous antibiotic therapy, based on their known microbiology, and consider adjunct treatment with tranexamic acid. Bronchial artery embolisation is the recommended first line treatment if significant haemoptysis persists. 


\section{Chest pain}

Chest pain is reported in studies of symptoms of bronchiectasis, but not often well described. King et al prospectively assessed chest pain experienced by 1787 patients with bronchiectasis over a 8 year follow-up period at the Monash Medical Centre. ${ }^{359}$ A careful assessment of the nature of the pain was made to test the hypothesis of visceral pain sensation related to bronchial inflammation. This bronchiectasis population was comparable to the UK population, except that a smaller proportion in Monash $(43 / 178)$ were taking inhaled corticosteroids than is suggested by the results of previous UK BTS bronchiectasis audits. ${ }^{166} 360$ 44 of the patients reported chest pain, and for 39 of these, the pain was associated with exacerbations. Only four had clear pleuritic pain, and 37 had other types of pain, with two having both pleuritic and other pain. The pain did appear to co-localise with the position of bronchiectasis on CT scan. It did not appear musculoskeletal related to coughing and was not associated with chest wall tenderness. It tended to occur early in the course of an exacerbation, and subside only towards the end of the recovery period. In 18 out of 33 patients who had imaging at the time of an exacerbation associated with chest pain, there were abnormalities such as consolidation, collapse or atelectasis. Only 13 subjects reported retrosternal discomfort associated with visceral airway pain receptors.

\section{Lung cancer}

A case series of post mortem specimens of 20 smokers and ex-smokers with peripheral nodular carcinoma of the lung showed that in 16 out of 20 cases the neoplasia arose out of clusters of non-segmental bronchiectasis. ${ }^{361}$ A database study of 57576 patients hospitalised with bronchiectasis between 1998 and 2010 in Taiwan showed an increased risk of lung cancer compared with a cohort of 230304 patients without bronchiectasis, adjusted for age, sex and comorbidities (adjusted $\mathrm{HR}=2.36,95 \% \mathrm{CI}=2.19$ to 2.35$).{ }^{362}$ Conversely a case-control study in South Korea of patients with moderate to severe COPD and lung cancer found that bronchiectasis was less prevalent in the patients with lung cancer than in matched controls without lung cancer (OR $0.25,95 \%$ CI 0.12 to 0.52 ), suggesting that the disease process in bronchiectasis may not increase the risk of lung cancer. ${ }^{363}$

\section{Vascular complications}

In a primary care database study, the rate of first coronary heart disease (CHD) and stroke were higher in people with bronchiectasis (HR for CHD 1.44 (95\% CI 1.27 to 1.63) and HR for stroke 1.71 (95\% CI 1.54 to 1.90$)$ ). ${ }^{364}$

Bronchiectasis severity (moderate or worse) has been shown to be an independent risk factor for vascular disease, ${ }^{365}$ with recurrent exacerbations (three or more per year) showing increased aortic stiffness. $^{366}$

A retrospective UK study examined the CT scans of 91 patients with bronchiectasis previously recruited for a quality of life study, and inferred the presence of pulmonary hypertension from measurement of the pulmonary arteriesand found there was an increased risk of death $(\mathrm{HR}, 1.24 ; 95 \% \mathrm{CI}, 1.13$ to 1.35 ; $\mathrm{P}<0.0001)$ in the presence of pulmonary hypertension in this study. ${ }^{367}$

Severe pulmonary hypertension was found in 12 patients from a prospective cross sectional study in Saudi Arabia of 94 patients with bronchiectasis but no known cardiac disease or cardiac risk factors, all with cystic bronchiectasis. Poorer ventilatory function was associated with increased risk of pulmonary hypertension. ${ }^{368}$

\section{Other}

In patients with bronchiectasis, depression and anxiety do occur at higher rates than in the healthy population. ${ }^{369-371}$ In a British study, there was a link between depression and exercise limitation due to breathlessness. ${ }^{369}$ In a Spanish study, using the Beck Depression Inventory and the State-Trait Anxiety Inventory, 34\% of patients had depression, $58.1 \%$ were above the $50^{\text {th }}$ percentile for state anxiety and $56.8 \%$ were above the $50 \%$ percentile for trait anxiety. $30 \%$ of patients had both anxiety and depression. Anxiety showed some correlation with sputum volume and bacterial colonisation, particularly $P$. aeruginosa, while depression appeared more common in women. ${ }^{372}$ A Korean study did not identify a link between mood disorders and specific clinical features of bronchiectasis. ${ }^{371}$

An important finding from an uncontrolled cross sectional study of 76 women attending a specialist bronchiectasis clinic in Newcastle-upon-Tyne was that $55 \%$ of women had symptoms of urinary incontinence. ${ }^{373}$

A study of systemic comorbidities included assessment of bone mineral density, which was not significantly different in patients with bronchiectasis and healthy controls matched for age, sex and smoking status. ${ }^{374}$

Corticosteroid responses in patients with bronchiectasis, of whom a high proportion were taking inhaled fluticasone, were found to be impaired in a cross-sectional analysis. ${ }^{176}$ Inhaled fluticasone treatment appeared to double the risk. Adrenal insufficiency was associated with poorer quality of life. This issue was addressed more fully in a case-control study of subjects with bronchiectasis and age- and sex-matched healthy controls, who were all subjected to low dose short synacthen tests. ${ }^{375}$ One patient with post-tuberculous bronchiectasis had absolute adrenal insufficiency and was treated with steroid replacement. In 38 patients with a valid baseline result, 15 had an impaired response to one microgram cosyntropin. The response was significantly better in controls, and was not affected by maintenance treatment with budesonide.

Nutritional deficiency has been noted incidentally in various studies $^{376}$ and was studied specifically by Olveira et al, but this study included both CF and non-CF bronchiectasis, which limits its applicability to this guideline. ${ }^{377}$

Fatigue and reduced exercise tolerance are commonly reported in bronchiectasis. In a UK study of 117 consecutive patients, routine clinical assessment included lung function, MRC dyspnoea score, sputum microbiology and an assessment of fatigue on the fatigue impact scale (FIS). ${ }^{378}$ Significant fatigue was found in $45 \%$ and was positively correlated with the MRC score $(r=0.57, \mathrm{P}<0.001)$ and negatively correlated with $\mathrm{FEV}_{1}$ $\%$ predicted $(\mathrm{r}=-0.30, \mathrm{P}=0.001)$. An Australian study identified fatigue in $74 \%$ of 103 patients with bronchiectasis. ${ }^{379}$

\section{SECTION 20}

\section{Summary of research recommendations}

See appendix 8 for details of the research recommendations in PICO format.

Consensus criteria for diagnosis of ABPA need to be validated in bronchiectasis cohorts.

Consensus criteria for definition of abnormal post pneumococcal test immunisation antibody responses need to be validated in bronchiectasis cohorts.

Randomised controlled trials using clinically important outcome measures are required to assess the effectiveness of airway clearance techniques in varying severities of bronchiectasis.

Randomised controlled trials are required to evaluate the effects of airway clearance techniques in patients who are undergoing an exacerbation. 
Randomised controlled trials are needed to assess the long term impact of muco-active therapies.

Randomised controlled trials are needed to assess the long term impact of anti-inflammatory therapies.

Long term randomised controlled trials of oral and inhaled antibiotics are needed to assess their efficacy and safety in patients with bronchiectasis who have frequent respiratory tract infections with recurrent $P$. aeruginosa infection or other potential pathogenic micro-organisms.

Further interventional/randomised controlled trials needed to establish the role of any alternative therapies in the management of bronchiectasis.

Studies assessing the benefits of nutritional supplementation in patients with bronchiectasis should be undertaken.

The role of education, self management plans and who delivers the pulmonary rehabilitation needs to be explored.

The role of pulmonary rehabilitation after exacerbations requiring hospital admission needs to be explored.

The incidence of cross-infection of respiratory pathogens in the group exercise setting should be investigated in the bronchiectasis population.

A randomised control trial of $P$. aeruginosa eradication therapy is needed to determine the microbiological and clinical outcomes of eradication therapy.

Randomised controlled trials are needed to assess which patients with bronchiectasis would benefit from long term Immunoglobulin $\mathrm{G}$ replacement therapy alone or as an adjunct to long term antibiotic therapy- assessing the optimal dose of IgG replacement and identification of ideal trough IgG level to prevent recurrent infections.

Large scale robust data that confirm or refute the transmissibility of key pathogens such as $P$. aeruginosa and non-tuberculous mycobacteria are needed.

\section{Author affiliations}

${ }^{1}$ Respiratory Medicine, Royal Infirmary of Edinburgh and University of Edinburgh, Edinburgh, UK

${ }^{2}$ Department of Respiratory Medicine, University Hospitals Birmingham NHS Foundation Trust (Queen Elizabeth Hospital), Birmingham, UK

${ }^{3}$ Scottish Centre for Respiratory Research, University of Dundee, Ninewells Hospital, Dundee, UK

${ }^{4}$ Institute of Cellular Medicine, NIHR Biomedical Research Centre for Aging and Freeman Hospital Adult Bronchiectasis service, Newcastle upon Tyne Hospitals NHS Foundation Trust, Newcastle, UK

${ }^{5}$ Royal Brompton Hospital and Imperial College London, and Queens University Belfast ${ }^{6}$ Department of Medicine, University of Cambridge, Cambridge UK

${ }^{7}$ Cambridge Centre for Lung Infection, Royal Papworth Hospital, Cambridge UK

${ }^{8}$ Royal Brompton Hospital, London, UK

${ }^{9}$ Box Surgery, Wiltshire, UK

${ }^{10}$ Department of Clinical Sciences, Brunel University London, London, UK

${ }^{11}$ Coventry, UK

${ }^{12}$ UCL Respiratory, University College London, London, UK

${ }^{13}$ Centre for Immunology and Vaccinology, Chelsea \&Westminster Hospital Campus, Department of Medicine, Imperial College London

${ }^{14}$ Host Defence Unit, Department of Respiratory Medicine, Royal Brompton Hospital and Harefield NHS Foundation Trust, London

${ }^{15}$ Chest \& Allergy Clinic St Mary's Hospital, Imperial College Healthcare NHS Trust

${ }^{16}$ University of Edinburgh MRC Centre for Inflammation Research, Edinburgh, UK

${ }^{17}$ Glenfield Hospital, Leicester, UK

${ }^{18}$ Department of Radiology, Royal Papworth Hospital, Cambridge UK

${ }^{19}$ University of Alberta, Edmonton, Alberta, Canada

${ }^{20}$ School of Pharmacy, Queens University Belfast, Belfast, UK

${ }^{21}$ Queen Elizabeth University Hospital, Glasgow, UK

Acknowledgements Guideline Development Group members are grateful to the members of the BTS Standards of Care Committee for their assistance during the production of the Guideline.

Contributors ATH, ALS and MRL chaired the guideline group, and as lead author led the drafting and revision of the document. All authors drafted sections of the guideline and undertook revisions of the paper. ATH, ALS and MRL had final responsibility for the guideline.

Competing interests All authors have completed a declaration of interest form in line with BTS Policy and copies are available from BTS head office on request. JDC has declared funding from Bayer Healthcare, Griffols, Pfizer, Insmed, Chiesi, Boehringer Ingelheim, GlaxoSmithkline, Astra Zeneca, Napp. ADS has declared funding from Bayer Healthcare, Pfizer, Insmed, Boehringer Ingelheim, Astra Zeneca. SE has declared funding from GlaxoSmithKline. AF has declared funding from GlaxoSmithKline, Zambon, Insmed, Verona. KGJ has declared funding from Boehringer Ingelheim, GlaxoSmithkline, Astra Zeneca, Napp, TEVA, Chiesi, Nutricia, Novartis. CSH has declared funding from Aradigm, Gilead, Griffols, Insmed, Chiesi, GlaxoSmithkline, TEVA, Vertex, Zambon, Novartis. ATH has declared funding from Bayer Healthcare, Boehringer Ingelheim. JRH has declared funding from Pfizer. MRL has declared funding from Bayer Healthcare, Insmed, Pulmocide, Griffols, Polyphor, Savara. MT has declared funding from Novartis, Basilea, Stragen. RW has declared funding from Bayer Healthcare.

Patient consent Not required.

Provenance and peer review Not commissioned; internally peer reviewed.

\section{REFERENCES}

1 Haworth C, Banks J, Capstick T, et al. BTS Guidelines for the management of nontuberculous mycobacterial pulmonary disease. Thorax 2017;72:1-64.

2 Seitz AE, Olivier KN, Steiner CA, et al. Trends and burden of bronchiectasis-associated hospitalizations in the United States, 1993-2006. Chest 2010;138:944-9.

3 Bibby S, Milne R, Beasley R. Hospital admissions for non-cystic fibrosis bronchiectasis in New Zealand. N Z Med J 2015;128:30-8.

4 Quint JK, Millett ER, Joshi M, et al. Changes in the incidence, prevalence and mortality of bronchiectasis in the UK from 2004 to 2013: a population-based cohort study. Eur Respir / 2016;47:186-93.

5 van der Bruggen-Bogaarts BA, van der Bruggen HM, van Waes PF, et al. Screening for bronchiectasis. A comparative study between chest radiography and highresolution CT. Chest 1996;109:608-11.

6 Young $\mathrm{K}$, Aspestrand F, Kolbenstvedt A. High resolution $\mathrm{CT}$ and bronchography in the assessment of bronchiectasis. Acta Radiol 1991;32:439-41.

7 Lucidarme 0 , Grenier P, Coche E, et al. Bronchiectasis: comparative assessment with thin-section CT and helical CT. Radiology 1996;200:673-9.

8 van der Bruggen-Bogaarts BA, van der Bruggen HM, van Waes PF, et al. Assessment of bronchiectasis: comparison of HRCT and spiral volumetric CT. J Comput Assist Tomogr 1996;20:15-19.

9 Dodd JD, Souza CA, Müller NL. Conventional high-resolution CT versus helical high-resolution MDCT in the detection of bronchiectasis. AJR Am J Roentgenol 2006; 187:414-20.

10 Jung KJ, Lee KS, Kim SY, et al. Low-dose, volumetric helical CT: image quality, radiation dose, and usefulness for evaluation of bronchiectasis. Invest Radiol 2000;35:557-63.

11 Del Donno M, Pavia D, Agnew JE, et al. Variability and reproducibility in the measurement of tracheobronchial clearance in healthy subjects and patients with different obstructive lung diseases. Eur Respir J 1988;1:613-20.

12 Currie DC, Pavia D, Agnew JE, et al. Impaired tracheobronchial clearance in bronchiectasis. Thorax 1987;42:126-30.

13 Ashford NS, Buxton-Thomas MS, Flower CD, et al. Aerosol lung scintigraphy in the detection of bronchiectasis. Clin Radiol 1988;39:29-32.

14 Nicotra MB, Rivera M, Dale AM, et al. Clinical, pathophysiologic, and microbiologic characterization of bronchiectasis in an aging cohort. Chest 1995;108:955-61.

15 King PT, Holdsworth SR, Freezer NJ, et al. Outcome in adult bronchiectasis. COPD 2005;2:27-34.

$16 \mathrm{Ni} \mathrm{Y}$, Shi G, Yu Y, et al. Clinical characteristics of patients with chronic obstructive pulmonary disease with comorbid bronchiectasis: a systemic review and metaanalysis. Int J Chron Obstruct Pulmon Dis 2015;10:1465-75.

17 Du Q, Jin J, Liu X, et al. Bronchiectasis as a Comorbidity of Chronic Obstructive Pulmonary Disease: A Systematic Review and Meta-Analysis. PLoS One 2016;11:e0150532.

18 Parr DG, Guest PG, Reynolds JH, et al. Prevalence and impact of bronchiectasis in alpha1-antitrypsin deficiency. Am J Respir Crit Care Med 2007;176:1215-21.

19 McMahon MA, O'Mahony MJ, O'Neill SJ, et al. Alpha-1 antitrypsin deficiency and computed tomography findings. J Comput Assist Tomogr 2005;29:549-53.

20 Ip MS, So SY, Lam WK, et al. High prevalence of asthma in patients with bronchiectasis in Hong Kong. Eur Respir J 1992;5:418-23.

21 Gupta S, Siddiqui S, Haldar P, et al. Qualitative analysis of high-resolution CT scans in severe asthma. Chest 2009;136:1521-8.

22 Luján $\mathrm{M}$, Gallardo X, Amengual MJ, et al. Prevalence of bronchiectasis in asthma according to oral steroid requirement: influence of immunoglobulin levels. Biomed Res Int 2013;2013:1-7.

23 Shoemark A, Ozerovitch L, Wilson R. Aetiology in adult patients with bronchiectasis. Respir Med 2007;101:1163-70. 
24 Shirahata Y. [Correlation between upper airway tract and lower airway tract in the break down of sinobronchiectasis]. Nihon Jibiinkoka Gakkai Kaiho 1990;93:1991-8.

25 Clausen E, Wittman C, Gingo M, et al. Chest computed tomography findings in HIVinfected individuals in the era of antiretroviral therapy. PLoS One 2014;9:e112237.

26 Okada F, Ando Y, Yoshitake S, et al. Pulmonary CT findings in 320 carriers of human T-lymphotropic virus type 1. Radiology 2006;240:559-64.

27 Honarbakhsh S, Taylor GP. High prevalence of bronchiectasis is linked to HTLV-1associated inflammatory disease. BMC Infect Dis 2015:15:258

28 Einsiedel L, Cassar O, Goeman E, et al. Higher human T-lymphotropic virus type 1 subtype C proviral loads are associated with bronchiectasis in indigenous australians: results of a case-control study. Open Forum Infect Dis 2014; 1:ofu023.

29 Skare TL, Nakano I, Escuissiato DL, et al. Pulmonary changes on high-resolution computed tomography of patients with rheumatoid arthritis and their association with clinical, demographic, serological and therapeutic variables. Rev Bras Reumatol 2011;51:325-7.

30 Leonel D, Lucia C, A M, et al. Pulmonary function test: its correlation with pulmonary high-resolution computed tomography in patients with rheumatoid arthritis. Rheumatol Int 2012;32:2111-6.

31 Mohd Noor N, Mohd Shahrir MS, Shahid MS, et al. Clinical and high resolution computed tomography characteristics of patients with rheumatoid arthritis lung disease. Int J Rheum Dis 2009;12:136-44.

32 Metafratzi ZM, Georgiadis AN, loannidou CV, et al. Pulmonary involvement in patients with early rheumatoid arthritis. Scand J Rheumatol 2007;36:338-44

33 Despaux J, Manzoni P, Toussirot E, et al. Prospective study of the prevalence of bronchiectasis in rheumatoid arthritis using high-resolution computed tomography. Rev Rhum Engl Ed 1998;65:453-61.

34 Perez T, Remy-Jardin M, Cortet B. Airways involvement in rheumatoid arthritis: clinical, functional, and HRCT findings. Am J Respir Crit Care Med 1998; 157:1658-65.

35 Cortet B, Flipo RM, Rémy-Jardin M, et al. Use of high resolution computed tomography of the lungs in patients with rheumatoid arthritis. Ann Rheum Dis 1995;54:815-9.

36 Tsuchiya Y, Takayanagi N, Sugiura $\mathrm{H}$, et al. Lung diseases directly associated with rheumatoid arthritis and their relationship to outcome. Eur Respir J 2011;37:1411-7

37 Zrour SH, Touzi M, Bejia I, et al. Correlations between high-resolution computed tomography of the chest and clinical function in patients with rheumatoid arthritis. Prospective study in 75 patients. Joint Bone Spine 2005;72:41-7.

38 Cortet B, Perez T, Roux N, et al. Pulmonary function tests and high resolution computed tomography of the lungs in patients with rheumatoid arthritis. Ann Rheum Dis 1997; 56:596-600.

39 Perry E, Eggleton P, De Soyza A, et al. Increased disease activity, severity and autoantibody positivity in rheumatoid arthritis patients with co-existent bronchiectasis. Int J Rheum Dis 2017:20.

40 Allain J, Saraux A, Guedes C, et al. Prevalence of symptomatic bronchiectasis in patients with rheumatoid arthritis. Rev Rhum Engl Ed 1997;64:531-7.

41 Soto-Cardenas MJ, Perez-De-Lis M, Bove A, et al. Bronchiectasis in primary Sjögren's syndrome: prevalence and clinical significance. Clin Exp Rheumatol 2010;28:647-53.

42 Yazisiz V, Arslan G, Ozbudak IH, et al. Lung involvement in patients with primary Sjögren's syndrome: what are the predictors? Rheumatol Int 2010:30:1317-24.

43 Lohrmann C, Uhl M, Warnatz K, et al. High-resolution CT imaging of the lung for patients with primary Sjogren's syndrome. Eur J Radio/ 2004;52:137-43.

44 Koyama M, Johkoh T, Honda O, et al. Pulmonary involvement in primary Sjögren's syndrome: spectrum of pulmonary abnormalities and computed tomography findings in 60 patients. J Thorac Imaging 2001;16:290-6.

45 Hwang HS, Yi CA, Yoo H, et al. The prevalence of bronchiectasis in patients with Marfan syndrome. Int J Tuberc Lung Dis 2014;18:995-7.

46 Yuksekkaya R, Almus F, Celıkyay F, et al. Pulmonary involvement in ankylosing spondylitis assessed by multidetector computed tomography. Pol J Radiol 2014;79:156-63.

47 El Maghraoui A, Dehhaoui M. Prevalence and characteristics of lung involvement on high resolution computed tomography in patients with ankylosing spondylitis: a systematic review. Pulm Med 2012;2012:1-5.

48 Sampaio-Barros PD, Cerqueira EM, Rezende SM, et al. Pulmonary involvement in ankylosing spondylitis. Clin Rheumatol 2007;26:225-30.

49 El Maghraoui A, Chaouir S, Abid A, et al. Lung findings on thoracic high-resolution computed tomography in patients with ankylosing spondylitis. Correlations with disease duration, clinical findings and pulmonary function testing. Clin Rheumato 2004;23:123-8.

50 Casserly IP, Fenlon HM, Breatnach $\mathrm{E}_{\text {, et }}$ al. Lung findings on high-resolution computed tomography in idiopathic ankylosing spondylitis--correlation with clinical findings, pulmonary function testing and plain radiography. $\mathrm{Br} J$ Rheumatol 1997:36:677-82

51 Fenlon HM, Casserly I, Sant SM, et al. Plain radiographs and thoracic highresolution $\mathrm{CT}$ in patients with ankylosing spondylitis. AJR Am J Roentgenol 1997; 168:1067-72
52 Fenlon HM, Doran M, Sant SM, et al. High-resolution chest CT in systemic lupus erythematosus. AJR Am J Roentgenol 1996:166:301-7.

53 Andonopoulos AP, Yarmenitis S, Georgiou P, et al. Bronchiectasis in systemic sclerosis. A study using high resolution computed tomography. Clin Exp Rheumatol 2001;19:187-90.

54 Phung S, Strange G, Chung LP, et al. Prevalence of pulmonary arterial hypertension in an Australian scleroderma population: screening allows for earlier diagnosis. Intern Med J 2009:39:682-91.

55 Desai $D$, Patil S, Udwadia Z, et al. Pulmonary manifestations in inflammatory bowel disease: a prospective study. Indian J Gastroenterol 2011;30:225-8.

56 Yilmaz A, Yilmaz Demirci N, Hoşgün D, et al. Pulmonary involvement in inflammatory bowel disease. World J Gastroenterol 2010;16:4952-7.

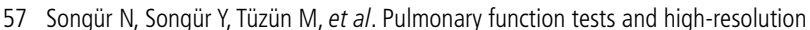
$\mathrm{CT}$ in the detection of pulmonary involvement in inflammatory bowel disease. J Clin Gastroenterol 2003:37:292-8.

58 Black H, Mendoza M, Murin S. Thoracic manifestations of inflammatory bowel disease. Chest 2007:131:524-32.

59 Kelly MG, Frizelle FA, Thornley PT, et al. Inflammatory bowel disease and the lung: is there a link between surgery and bronchiectasis? Int J Colorectal Dis 2006;21:754-7

60 Mahadeva R, Walsh G, Flower CD, et al. Clinical and radiological characteristics of lung disease in inflammatory bowel disease. Eur Respir J 2000;15:41-8.

61 Morice AH, McGarvey L, Pavord I, et al. Recommendations for the management of cough in adults. Thorax 2006:61:i1-24.

62 Pasteur MC, Helliwell SM, Houghton SJ, et al. An investigation into causative factors in patients with bronchiectasis. Am J Respir Crit Care Med 2000;162:1277-84

63 Lonni S, Chalmers JD, Goeminne PC, et al. Etiology of Non-Cystic Fibrosis Bronchiectasis in Adults and Its Correlation to Disease Severity. Ann Am Thorac Soc 2015;12:1764-70.

64 Anwar GA, McDonnell MJ, Worthy SA, et al. Phenotyping adults with non-cystic fibrosis bronchiectasis: a prospective observational cohort study. Respir Med 2013:107:1001-7.

65 McShane PJ, Naureckas ET, Strek ME. Bronchiectasis in a diverse US population: effects of ethnicity on etiology and sputum culture. Chest 2012;142:159-67

66 Altenburg J, Wortel K, van der Werf TS, et al. Non-cystic fibrosis bronchiectasis: clinical presentation, diagnosis and treatment, illustrated by data from a Dutch Teaching Hospital. Neth J Med 2015:73:147-54.

67 Gao YH, Guan WJ, Liu SX, et al. Aetiology of bronchiectasis in adults: A systematic literature review. Respirology 2016;21:1376-83.

68 Kelly MG, Murphy S, Elborn JS. Bronchiectasis in secondary care: a comprehensive profile of a neglected disease. Eur J Intern Med 2003;14:488-92.

69 Bahous J, Malo JL, Paquin R, et al. Allergic bronchopulmonary aspergillosis and sensitization to Aspergillus fumigatus in chronic bronchiectasis in adults. Clin Allergy 1985; 15:571-9.

70 Stevens DA, Moss RB, Kurup VP, et al. Allergic bronchopulmonary aspergillosis in cystic fibrosis--state of the art: Cystic Fibrosis Foundation Consensus Conference. Clin Infect Dis 2003;37 Suppl 3:S225-S264.

71 Rosenberg M, Patterson R, Mintzer R, et al. Clinical and immunologic criteria for the diagnosis of allergic bronchopulmonary aspergillosis. Ann Intern Med 1977:86:405-14

72 Patterson R, Greenberger PA, Halwig JM, et al. Allergic bronchopulmonary aspergillosis. Natural history and classification of early disease by serologic and roentgenographic studies. Arch Intern Med 1986;146:916-8.

73 Agarwal R, Chakrabarti A, Shah A, et al. Allergic bronchopulmonary aspergillosis: review of literature and proposal of new diagnostic and classification criteria. Clin Exp Allergy 2013;43:850-73.

74 Agarwal R, Gupta D, Aggarwal AN, et al. Clinical significance of hyperattenuating mucoid impaction in allergic bronchopulmonary aspergillosis: an analysis of 155 patients. Chest 2007;132:1183-90.

75 Li AM, Sonnappa S, Lex C, et al. Non-CF bronchiectasis: does knowing the aetiology lead to changes in management? Eur Respir J 2005;26:8-14.

76 Qi Q, Wang W, Li T, et al. Aetiology and clinical characteristics of patients with bronchiectasis in a Chinese Han population: A prospective study. Respirology 2015;20:917-24.

77 Agarwal R, Aggarwal AN, Gupta D, et al. Aspergillus hypersensitivity and allergic bronchopulmonary aspergillosis in patients with bronchial asthma: systematic review and meta-analysis. Int J Tuberc Lung Dis 2009;13:936-44.

78 Agarwal R, Maskey D, Aggarwal AN, et al. Diagnostic performance of various tests and criteria employed in allergic bronchopulmonary aspergillosis: a latent class analysis. PLoS One 2013:8:e61105.

79 Agarwal R, Aggarwal AN, Sehgal IS, et al. Utility of IgE (total and Aspergillus fumigatus specific) in monitoring for response and exacerbations in allergic bronchopulmonary aspergillosis. Mycoses 2016:59:1-6.

80 Agarwal R, Khan A, Aggarwal AN, et al. Clinical relevance of peripheral blood eosinophil count in allergic bronchopulmonary aspergillosis. J Infect Public Health 2011;4:235-43 
81 Agarwal R, Gupta D, Aggarwal AN, et al. Allergic bronchopulmonary aspergillosis: lessons from 126 patients attending a chest clinic in north India. Chest 2006;130:442-8.

82 Campbell MJ, Clayton YM. Bronchopulmonary aspergillosis. a correlation of the clinical and laboratory findings in 272 patients investigated for bronchopulmonary aspergillosis. Am Rev Respir Dis 1964;89:186-96.

83 Chakrabarti A, Sethi S, Raman DS, et al. Eight-year study of allergic bronchopulmonary aspergillosis in an Indian teaching hospital. Mycoses 2002;45:295-9.

84 Baxter CG, Denning DW, Jones AM, et al. Performance of two Aspergillus IgG EIA assays compared with the precipitin test in chronic and allergic aspergillosis. Clin Microbiol Infect 2013;19:E197-204.

85 Pashley $\mathrm{CH}$, Fairs A, Morley JP, et al. Routine processing procedures for isolating filamentous fungi from respiratory sputum samples may underestimate fungal prevalence. Med Mycol 2012;50:433-8.

86 Jolles $\mathrm{S}$. The variable in common variable immunodeficiency: a disease of complex phenotypes. J Allergy Clin Immunol Pract 2013;1:545-56.

87 Dhalla F, Misbah SA. Secondary antibody deficiencies. Curr Opin Allergy Clin Immunol 2015:15:505-13.

88 Kyle RA, Rajkumar SV. Management of monoclonal gammopathy of undetermined significance (MGUS) and smoldering multiple myeloma (SMM). Oncology 2011;25:578-86.

89 Glavey SV, Leung N. Monoclonal gammopathy: The good, the bad and the ugly. Blood Rev 2016;30:223-31.

90 Guan WJ, Gao YH, Xu G, et al. Aetiology of bronchiectasis in Guangzhou, southern China. Respirology 2015:20:739-48.

91 Ameratunga R, Brewerton M, Slade C, et al. Comparison of diagnostic criteria for common variable immunodeficiency disorder. Front Immuno/ 2014;5:415.

92 Orange JS, Ballow M, Stiehm ER, et al. Use and interpretation of diagnostic vaccination in primary immunodeficiency: a working group report of the Basic and Clinical Immunology Interest Section of the American Academy of Allergy, Asthma \& Immunology. J Allergy Clin Immunol 2012;130:S1-24.

93 Vendrell M, de Gracia J, Rodrigo MJ, et al. Antibody production deficiency with normal IgG levels in bronchiectasis of unknown etiology. Chest 2005;127:197-204

94 Paris K, Sorensen RU. Assessment and clinical interpretation of polysaccharide antibody responses. Ann Allergy Asthma Immunol 2007;99:462-4.

95 Bonilla FA, Khan DA, Ballas ZK, et al. Practice parameter for the diagnosis and management of primary immunodeficiency. J Allergy Clin Immunol 2015:136:1186-205.

96 Bonilla FA, Barlan I, Chapel H, et al. International Consensus Document (ICON) Common Variable Immunodeficiency Disorders. J Allergy Clin Immunol Pract 2016:4:38-59.

97 Perry E, Stenton C, Kelly C, et al. RA autoantibodies as predictors of rheumatoid arthritis in non-cystic fibrosis bronchiectasis patients. Eur Respir 2014;44:1082-5.

98 Mandal P, Morice AH, Chalmers JD, et al. Symptoms of airway reflux predict exacerbations and quality of life in bronchiectasis. Respir Med 2013;107:1008-13.

99 Hu ZW, Wang ZG, Zhang Y, et al. Gastroesophageal reflux in bronchiectasis and the effect of anti-reflux treatment. BMC Pulm Med 2013;13:34.

100 Li N, Pei P, Bu DF, et al. A novel CFTR mutation found in a Chinese patient with cystic fibrosis. Chin Med J 2006;119:103-9.

101 Li W, Sun L, Corey M, et al. Understanding the population structure of North American patients with cystic fibrosis. Clin Genet 2011;79:136-46.

102 De Boeck K, Wilschanski M, Castellani C, et al. Cystic fibrosis: terminology and diagnostic algorithms. Thorax 2006;61:627-35

103 Farrell PM, White TB, Ren CL, et al. Diagnosis of cystic fibrosis: consensus guidelines from the cystic fibrosis foundation. J Pediatr 2017:181:S4-1.

104 Hubert D, Fajac I, Bienvenu T, et al. Diagnosis of cystic fibrosis in adults with diffuse bronchiectasis. Journal of Cystic Fibrosis 2004;3:203.

105 NICE. Cystic fibrosis: diagnosis and management. London: NICE, 2017

106 Lucas JS, Barbato A, Collins SA, et al. European Respiratory Society guidelines for the diagnosis of primary ciliary dyskinesia. Eur Respir J 2017;49:1601090.

107 Lundberg JO, Weitzberg E, Nordvall SL, et al. Primarily nasal origin of exhaled nitric oxide and absence in Kartagener's syndrome. Eur Respir 1994; 7:1501-4.

108 Noone PG, Leigh MW, Sannuti A, et al. Primary ciliary dyskinesia: diagnostic and phenotypic features. Am J Respir Crit Care Med 2004;169:459-67.

109 Karadag B, James AJ, Gültekin E, et al. Nasal and lower airway level of nitric oxide in children with primary ciliary dyskinesia. Eur Respir J 1999;13:1402-5.

110 Jackson CL, Behan L, Collins SA, et al. Accuracy of diagnostic testing in primary ciliary dyskinesia. Eur Respir J 2016;47:837-48.

111 Horváth I, Loukides S, Wodehouse T, et al. Comparison of exhaled and nasal nitric oxide and exhaled carbon monoxide levels in bronchiectatic patients with and without primary ciliary dyskinesia. Thorax 2003;58:68-72.

112 Young D. Surgical treatment of male infertility. Reproduction 1970;23:541-2.

113 Friedman KJ, Teichtahl H, De Kretser DM, et al. Screening Young syndrome patients for CFTR mutations. Am J Respir Crit Care Med 1995;152:1353-7.
114 Greenstone MA, Rutman A, Hendry WF, et al. Ciliary function in Young's syndrome. Thorax 1988;43:153-4.

115 de longh R, Ing A, Rutland J. Mucociliary function, ciliary ultrastructure, and ciliary orientation in Young's syndrome. Thorax 1992;47:184-7.

116 Hendry WF, A'Hern RP, Cole PJ. Was Young's syndrome caused by exposure to mercury in childhood? BMJ 1993;307:1579-82.

117 Di Scioscio V, Zompatori M, Mistura I, et al. The role of spiral multidetector dynamic CT in the study of Williams-Campbell syndrome. Acta Radiol 2006; $47: 798-800$

118 Dunne MG, Reiner B. CT features of tracheobronchomegaly. J Comput Assist Tomogr 1988;12:388-91.

119 Martínez-García MÁ, de Gracia J, Vendrell Relat M, et al. Multidimensional approach to non-cystic fibrosis bronchiectasis: the FACED score. Eur Respir 2014;43:1357-67.

120 Chalmers JD, Goeminne P, Aliberti S, et al. The bronchiectasis severity index An international derivation and validation study. Am J Respir Crit Care Med 2014;189:576-85.

121 McDonnell MJ, Aliberti S, Goeminne PC, et al. Multidimensional severity assessment in bronchiectasis: an analysis of seven European cohorts. Thorax 2016;71:1110-8.

122 Rosales-Mayor E, Polverino E, Raguer L, et al. Comparison of two prognostic scores (BSI and FACED) in a Spanish cohort of adult patients with bronchiectasis and improvement of the FACED predictive capacity for exacerbations. PLoS One 2017; 12:e0175171

123 Martinez-Garcia MA, Athanazio RA, Girón R, et al. Predicting high risk of exacerbations in bronchiectasis: the E-FACED score. Int $J$ Chron Obstruct Pulmon Dis 2017:12:275-84.

124 Murray MP, Pentland JL, Hill AT. A randomised crossover trial of chest physiotherapy in non-cystic fibrosis bronchiectasis. Eur Respir J 2009:34:1086-92.

125 Physiotherapy and NIPPV. In: Bott J, Moran F, A.K. S, eds. Non-invasive respiratory support. London: Chapman and Hall, 1995:133-42.

126 Dodd ME, Bronchiectasis WK. primary ciliary dyskinesia and cystic fibrosis. In: PJaPS A, ed. Physiotherapy for Respiratory and Cardiac Problems Adults and Paediatrics. 4th edn. Churchill Llvingstone: Elsevier, 2008:550-89.

127 Raj AA, Pavord DI, Birring SS. Clinical cough IV:what is the minimal important difference for the Leicester Cough Questionnaire? Handb Exp Pharmacol 2009;187:311-20.

128 Mutalithas K, Watkin G, Willig B, et al. Improvement in health status following bronchopulmonary hygiene physical therapy in patients with bronchiectasis. Respir Med 2008:102:1140-4.

129 McCullough AR, Tunney MM, Stuart Elborn J, et al. Predictors of adherence to treatment in bronchiectasis. Respir Med 2015;109:838-45.

130 Flude LJ, Agent P, Bilton D. Chest physiotherapy techniques in bronchiectasis. Clin Chest Med 2012:33:351-61.

131 O'Neill B, Bradley JM, McArdle N, et al. The current physiotherapy management of patients with bronchiectasis: a UK survey. Int J Clin Pract 2002;56:34-5.

132 Syed N, Maiya AG, Siva Kumar T. Active Cycles of Breathing Technique (ACBT) versus conventional chest physical therapy on airway clearance in bronchiectasis - a crossover trial. Adv Physiother 2009;11:193-8.

133 Thompson CS, et al. Randomised crossover study of the Flutter device and the active cycle of breathing technique in non-cystic fibrosis bronchiectasis. Thorax 2002:57:446-8

134 Patterson JE, Bradley JM, Hewitt O, et al. Airway clearance in bronchiectasis: a randomized crossover trial of active cycle of breathing techniques versus Acapella. Respiration 2005;72:239-42.

135 Eaton T, Young P, Zeng I, et al. A randomized evaluation of the acute efficacy, acceptability and tolerability of Flutter and active cycle of breathing with and without postural drainage in non-cystic fibrosis bronchiectasis. Chron Respir Dis 2007;4:23-30.

136 Patterson JE, Bradley JM, Elborn JS. Airway clearance in bronchiectasis: a randomized crossover trial of active cycle of breathing techniques (incorporating postural drainage and vibration) versus test of incremental respiratory endurance. Chron Respir Dis 2004;1:127-30

137 AbdelHalim HA, AboEINaga HH, Fathy KA. Comparison between active cycles of breathing with postural drainage versus conventional chest physiotherapy in subjects with bronchiectasis. Egypt J Chest Dis Tuberc 2016;65:157-65.

138 Main E, Grillo L, Rand S. Airway clearance strategies in cystic fibrosis and non-cystic fibrosis bronchiectasis. Semin Respir Crit Care Med 2015;36:251-66.

139 Ramos EM, Ramos D, Moreira GL, et al. Viscoelastic properties of bronchial mucus after respiratory physiotherapy in subjects with bronchiectasis. Respir Care 2015:60:724-30

140 Lee AL, Denehy L, Wilson JW, et al. Upright positive expiratory pressure therapy and exercise: effects on gastroesophageal reflux in COPD and bronchiectasis. Respir Care 2012:57:1460-7.

141 Volsko TA, DiFiore J, Chatburn RL. Performance comparison of two oscillating positive expiratory pressure devices: Acapella versus Flutter. Respir Care 2003;48:124-30

142 Tsang SMH, Jones AYM. Postural drainage or flutter $®$ device in conjunction with breathing and coughing compared to breathing and coughing alone 
in improving secretion removal and lung function in patients with acute exacerbation of bronchiectasis: a pilot study. Hong Kong Physiotherapy Journal 2003;21:29-36.

143 Svenningsen S, Guo F, McCormack DG, et al. Noncystic fibrosis bronchiectasis: regional abnormalities and response to airway clearance therapy using pulmonary functional magnetic resonance imaging. Acad Radio/ 2017;24:4-12.

144 Lee AL, Williamson HC, Lorensini S, et al. The effects of oscillating positive expiratory pressure therapy in adults with stable non-cystic fibrosis bronchiectasis: a systematic review. Chron Respir Dis 2015;12:36-46.

145 Herrero-Cortina B, Vilaró J, Martí D, et al. Short-term effects of three slow expiratory airway clearance techniques in patients with bronchiectasis: a randomised crossover trial. Physiotherapy 2016;102:357-64.

146 Naraparaju S, Vaishali K, Venkatesan P, et al. A comparison of the Acapella and a threshold inspiratory muscle trainer for sputum clearance in bronchiectasis-A pilot study. Physiother Theory Pract 2010;26:353-7.

147 Paneroni M, Clini E, Simonelli C, et al. Safety and efficacy of short-term intrapulmonary percussive ventilation in patients with bronchiectasis. Respir Care 2011;56:984-8.

148 Sukumalchantra Y, Park SS, Williams MH. The effect of intermittent positive pressure breathing (IPPB) in acute ventilatory failure. Am Rev Respir Dis 1965;92:885-93.

149 Cl S, Chang CC, Lin YK, et al. Randomized Crossover Study of Lung Expansion Therapy Using Negative Pressure and Positive Pressure in Bronchiectasis. J Clin Exp Med 2012:4:149-53.

150 Lee AL, Hill CJ, Cecins N, et al. The short and long term effects of exercise training in non-cystic fibrosis bronchiectasis--a randomised controlled trial. Respir Res 2014; 15:44

151 Wills PJ, Wodehouse T, Corkery K, et al. Short-term recombinant human DNase in bronchiectasis. Effect on clinical state and in vitro sputum transportability. Am J Respir Crit Care Med 1996:154:413-7.

152 O'Donnell AE, Barker AF, llowite JS, et al. Treatment of idiopathic bronchiectasis with aerosolized recombinant human DNase I. rhDNase Study Group. Chest 1998:113:1329-34.

153 Sutton PP, Gemmell HG, Innes N, et al. Use of nebulised saline and nebulised terbutaline as an adjunct to chest physiotherapy. Thorax 1988;43:57-60.

154 Conway JH, Fleming JS, Perring $S$, et al. Humidification as an adjunct to chest physiotherapy in aiding tracheo-bronchial clearance in patients with bronchiectasis. Respir Med 1992;86:109-14.

155 Kellett F, Robert NM. Nebulised 7\% hypertonic saline improves lung function and quality of life in bronchiectasis. Respir Med 2011;105:1831-5.

156 Bradley JM, Treacy K, O'Neill B, et al. S106 A randomised double blind 13 week crossover trial of hypertonic saline (HTS) $(6 \%)$ vs isotonic saline (ITS) $(0.9 \%)$ in patients with bronchiectasis. Thorax 2011;66:A49.

157 Nicolson $\mathrm{CH}$, Stirling RG, Borg BM, et al. The long term effect of inhaled hypertonic saline 6\% in non-cystic fibrosis bronchiectasis. Respir Med 2012;106:661-7.

158 Kellett F, Redfern J, Niven RM. Evaluation of nebulised hypertonic saline (7\%) as an adjunct to physiotherapy in patients with stable bronchiectasis. Respir Med 2005;99:27-31.

159 Bilton D, Daviskas E, Anderson SD, et al. Phase 3 randomized study of the efficacy and safety of inhaled dry powder mannitol for the symptomatic treatment of non-cystic fibrosis bronchiectasis. Chest 2013;144:215-25.

160 Bilton D, Tino G, Barker AF, et al. Inhaled mannitol for non-cystic fibrosis bronchiectasis: a randomised, controlled trial. Thorax 2014;69:1073-9.

161 Crisafulli $\mathrm{E}$, Coletti $\mathrm{O}$, Costi S, et al. Effectiveness of erdosteine in elderly patients with bronchiectasis and hypersecretion: a 15-day, prospective, parallel, open-label, pilot study. Clin Ther 2007;29:2001-9.

162 Olivieri D, Ciaccia A, Marangio E, et al. Role of bromhexine in exacerbations of bronchiectasis. Double-blind randomized multicenter study versus placebo. Respiration 1991;58:117-21.

163 Hasani A, Chapman TH, McCool D, et al. Domiciliary humidification improves lung mucociliary clearance in patients with bronchiectasis. Chron Respir Dis 2008:5:81-6.

164 Briffa PJ, Anderson SD, Burton DL, et al. Sodium cromoglycate and eformoterol attenuate sensitivity and reactivity to inhaled mannitol in subjects with bronchiectasis. Respirology 2011;16:161-6.

165 Elkins MR, Anderson SD, Perry CP, et al. Inspiratory flows and volumes in subjects with non-cf bronchiectasis using a new dry powder inhaler device. Open Respir Med J 2014;8:8-13

166 Hill AT, Welham S, Reid K, et al. British Thoracic Society national bronchiectasis audit 2010 and 2011. Thorax 2012;67:928-30.

167 Fuschillo S, De Felice A, Balzano G. Mucosal inflammation in idiopathic bronchiectasis: cellular and molecular mechanisms. Eur Respir J 2008;31:396-406.

168 Chalmers JD, Smith MP, McHugh BJ, et al. Short- and long-term antibiotic treatment reduces airway and systemic inflammation in non-cystic fibrosis bronchiectasis. Am $J$ Respir Crit Care Med 2012;186:657-65.

169 Kapur N, Bell S, Kolbe J, et al. Inhaled steroids for bronchiectasis. Cochrane Database Syst Rev 2009;86:CD000996

170 Elborn JS, Johnston B, Allen F, et al. Inhaled steroids in patients with bronchiectasis. Respir Med 1992;86:121-4.
171 Joshi JM, Sundaram P. Role of inhaled steroids in stable Bronchiectasis. Indian practitioner 2004;57 http://onlinelibrary.wiley.com/o/cochrane/clcentral/articles/723/ CN-00507723/frame.html

172 Martínez-García MA, Perpiñá-Tordera M, Román-Sánchez P, et al. Inhaled steroids improve quality of life in patients with steady-state bronchiectasis. Respir Med 2006;100:1623-32.

173 Tsang KW, Ho PL, Lam WK, et al. Inhaled fluticasone reduces sputum inflammatory indices in severe bronchiectasis. Am J Respir Crit Care Med 1998;158:723-7.

174 Tsang KW, Tan KC, Ho PL, et al. Exhaled nitric oxide in bronchiectasis: the effects of inhaled corticosteroid therapy. Int J Tuberc Lung Dis 2004;8:1301-7.

175 Tsang KW, Tan KC, Ho PL, et al. Inhaled fluticasone in bronchiectasis: a 12 month study. Thorax 2005;60:239-43.

176 Holme J, Tomlinson JW, Stockley RA, et al. Adrenal suppression in bronchiectasis and the impact of inhaled corticosteroids. Eur Respir J 2008;32:1047-52.

177 Martínez-García MÁ, Soler-Cataluña JJ, Catalán-Serra P, et al. Clinical efficacy and safety of budesonide-formoterol in non-cystic fibrosis bronchiectasis. Chest 2012:141:461-8

178 Lasserson T, Holt K, Greenstone M. Oral steroids for bronchiectasis (stable and acute exacerbations). Cochrane Database Syst Rev 2001:4:CD002162.

179 Steele K, Greenstone M, Lasserson JA. Oral methyl-xanthines for bronchiectasis. Cochrane Database Syst Rev 2001;1:CD002734.

180 Pizzutto SJ, Upham JW, Yerkovich ST, et al. Inhaled non-steroid anti-inflammatories for children and adults with bronchiectasis. Cochrane Database Syst Rev 2010:4:CD007525.

181 Corless JA, Warburton CJ. Leukotriene receptor antagonists for non-cystic fibrosis bronchiectasis. Cochrane Database Syst Rev 2000;157:CD002174.

182 Chalmers JD, Hill AT. Mechanisms of immune dysfunction and bacterial persistence in non-cystic fibrosis bronchiectasis. Mol Immunol 2013:55:27-34.

183 Stockley R, De Soyza A, Gunawardena K, et al. Phase II study of a neutrophil elastase inhibitor (AZD9668) in patients with bronchiectasis. Respir Med 2013;107:524-33.

184 De Soyza A, Pavord I, Elborn JS, et al. A randomised, placebo-controlled study of the CXCR2 antagonist AZD5069 in bronchiectasis. Eur Respir J 2015;46:1021-32.

185 Mandal P, Chalmers JD, Graham C, et al. Atorvastatin as a stable treatment in bronchiectasis: a randomised controlled trial. Lancet Respir Med 2014;2:455-63.

186 Bedi P, Chalmers JD, Graham C, et al. A Randomized Controlled Trial of Atorvastatin in Patients With Bronchiectasis Infected With Pseudomonas Aeruginosa: A Proof of Concept Study. Chest 2017;152:368-78.

187 Anonymous. PROLONGED antibiotic treatment of severe bronchiectasis; a report by a subcommittee of the Antibiotics Clinical Trials (non-tuberculous) Committee of the Medical Research Council. Br Med J 1957;2:255-9.

188 Cherniack NS, Vosti KL, Dowling HF, et al. Long-term treatment of bronchlectasis and chronic bronchitis; a controlled study of the effects of tetracycline, penicillin, and an oleandomycinpenicillin mixture. AMA Arch Intern Med 1959;103:345-53.

189 Currie DC, Garbett ND, Chan KL, et al. Double-blind randomized study of prolonged higher-dose oral amoxycillin in purulent bronchiectasis. Q J Med 1990;76:799-816.

190 Wong C, Jayaram L, Karalus N, et al. Azithromycin for prevention of exacerbations in non-cystic fibrosis bronchiectasis (EMBRACE): a randomised, double-blind, placebo-controlled trial. Lancet 2012;380:660-7.

191 Altenburg J, de Graaff CS, Stienstra Y, et al. Effect of azithromycin maintenance treatment on infectious exacerbations among patients with non-cystic fibrosis bronchiectasis: the BAT randomized controlled trial. JAMA 2013·309:1251-9.

192 Serisier DJ, Martin ML, McGuckin MA, et al. Effect of long-term, low-dose erythromycin on pulmonary exacerbations among patients with non-cystic Fibrosis Bronchiectasis. JAMA 2013;309:1260-7.

193 Shi ZL, Peng H, Hu XW, et al. Effectiveness and safety of macrolides in bronchiectasis patients: a meta-analysis and systematic review. Pulm Pharmacol Ther 2014:28.

194 Gao YH, Guan WJ, Xu G, et al. Macrolide therapy in adults and children with non-cystic fibrosis bronchiectasis: a systematic review and meta-analysis. PLoS One 2014;9:e90047.

195 Zhuo GY, He Q, Xiang-Lian L, et al. Prolonged treatment with macrolides in adult patients with non-cystic fibrosis bronchiectasis: meta-analysis of randomized controlled trials. Pulm Pharmacol Ther 2014;29:80-8.

196 Wu Q, Shen W, Cheng H, et al. Long-term macrolides for non-cystic fibrosis bronchiectasis: a systematic review and meta-analysis. Respirology 2014;19:321-9.

197 Rogers GB, Bruce KD, Martin ML, et al. The effect of long-term macrolide treatment on respiratory microbiota composition in non-cystic fibrosis bronchiectasis: an analysis from the randomised, double-blind, placebo-controlled BLESS trial. Lancet Respir Med 2014:2:988-96.

198 Wilson R, Welte T, Polverino E, et al. Ciprofloxacin dry powder for inhalation in non-cystic fibrosis bronchiectasis: a phase II randomised study. Eur Respir J 2013:41:1107-15.

199 Serisier DJ, Bilton D, De Soyza A, et al. Inhaled, dual release liposomal ciprofloxacin in non-cystic fibrosis bronchiectasis (ORBIT-2): a randomised, double-blind, placebo-controlled trial. Thorax 2013;68:812-7. 
200 Haworth CS, Foweraker JE, Wilkinson P, et al. Inhaled colistin in patients with bronchiectasis and chronic Pseudomonas aeruginosa infection. Am J Respir Crit Care Med 2014;189:975-82.

201 Murray MP, Govan JR, Doherty CJ, et al. A randomized controlled trial of nebulized gentamicin in non-cystic fibrosis bronchiectasis. Am J Respir Crit Care Med 2011;183:491-9.

202 Barker AF, O'Donnell AE, Flume $P$, et al. Aztreonam for inhalation solution in patients with non-cystic fibrosis bronchiectasis (AIR-BX1 and AIR-BX2): two randomised double-blind, placebo-controlled phase 3 trials. Lancet Respir Med 2014:2:738-49.

203 De Soyza A, Aksamit T, Bandel TJ, et al. RESPIRE 1: a phase III placebo-controlled randomised trial of ciprofloxacin dry powder for inhalation in non-cystic fibrosis bronchiectasis. Eur Respir J 2018;51:1702052.

204 Aksamit T, De Soyza A, Bandel TJ, et al. RESPIRE 2: a phase III placebo-controlled randomised trial of ciprofloxacin dry powder for inhalation in non-cystic fibrosis bronchiectasis. Eur Respir J 2018;51:1702053.

205 Mandal P, Sidhu MK, Donaldson LS, et al. Eight-weekly intravenous antibiotics is beneficial in severe bronchiectasis. QJM 2013;106:27-33.

206 Hnin K, Nguyen C, Carson KV, et al. Prolonged antibiotics for non-cystic fibrosis bronchiectasis in children and adults. Cochrane Database Syst Rev 2015;209:CD001392.

207 Yang JW, Fan LC, Lu HW, et al. Efficacy and safety of long-term inhaled antibiotic for patients with noncystic fibrosis bronchiectasis: a meta-analysis. Clin Respir J 2016:10:731-9.

208 Goyal V, Chang AB. Combination inhaled corticosteroids and long-acting beta2agonists for children and adults with bronchiectasis. Cochrane Database Syst Rev 2014;1:CD010327.

209 Lasserson T, Holt K, Evans D, et al. Anticholinergic therapy for bronchiectasis. Cochrane Database Syst Rev 2001;4:CD002163.

210 Nogrady SG, Evans WV, Davies BH. Reversibility of airways obstruction in bronchiectasis. Thorax 1978;33:635-7.

211 Franco F, Sheikh A, Greenstone M. Short acting beta2-agonists for bronchiectasis. Cochrane Database Syst Rev 2003;18.

212 Bradley JM, Wilson JJ, Hayes K, et al. Sedentary behaviour and physical activity in bronchiectasis: a cross-sectional study. BMC Pulm Med 2015;15:61.

213 O'Donovan G, Blazevich AJ, Boreham C, et al. The ABC of Physical Activity for Health: a consensus statement from the British Association of Sport and Exercise Sciences. $J$ Sports Sci 2010;28:573-91.

214 Newall C, Stockley RA, Hill SL. Exercise training and inspiratory muscle training in patients with bronchiectasis. Thorax 2005;60:943-8.

215 Mandal P, Sidhu MK, Kope L, et al. A pilot study of pulmonary rehabilitation and chest physiotherapy versus chest physiotherapy alone in bronchiectasis. Respir Med 2012;106:1647-54.

216 Olveira G, Olveira C, Doña E, et al. Oral supplement enriched in HMB combined with pulmonary rehabilitation improves body composition and health related quality of life in patients with bronchiectasis (Prospective, Randomised Study). Clin Nutr 2016;35:S23.

217 van Zeller M, Mota PC, Amorim A, et al. Pulmonary rehabilitation in patients with bronchiectasis: pulmonary function, arterial blood gases, and the 6-minute walk test. J Cardiopulm Rehabil Prev 2012;32:278-83.

218 Zanini A, Aiello M, Adamo D, et al. Effects of Pulmonary Rehabilitation in Patients with Non-Cystic Fibrosis Bronchiectasis: A Retrospective Analysis of Clinical and Functional Predictors of Efficacy. Respiration 2015;89:525-33.

219 Liaw MY, Wang YH, Tsai YC, et al. Inspiratory muscle training in bronchiectasis patients: a prospective randomized controlled study. Clin Rehabil 2011;25:524-36

220 Lee AL, Cecins N, Holland AE, et al. Field Walking Tests Are Reliable and Responsive to Exercise Training in People With Non-Cystic Fibrosis Bronchiectasis. J Cardiopulm Rehabil Prev 2015:35:439-45.

221 Lee AL, Hill CJ, Cecins N, et al. Minimal important difference in field walking tests in non-cystic fibrosis bronchiectasis following exercise training. Respir Med 2014;108:1303-9.

222 Al-Refaie RE, Amer S, El-Shabrawy M. Surgical treatment of bronchiectasis: a retrospective observational study of 138 patients. J Thorac Dis 2013;5:228-33.

223 Cobanoglu U, Yalcinkaya I, Er M, et al. Surgery for bronchiectasis: The effect of morphological types to prognosis. Ann Thorac Med 2011;6:25-32.

224 Halezeroglu S, Keles M, Uysal A, et al. Factors affecting postoperative morbidity and mortality in destroyed lung. Ann Thorac Surg 1997;64:1635-8.

225 Agasthian T, Deschamps C, Trastek VF, et al. Surgical management of bronchiectasis. Ann Thorac Surg 1996;62:976-80. Discussion 79-80.

226 Smit HJ, Schreurs AJ, Van den Bosch JM, et al. Is resection of bronchiectasis beneficial in patients with primary ciliary dyskinesia? Chest 1996;109:1541-4.

227 Ashour M, Al-Kattan KM, Jain SK, et al. Surgery for unilateral bronchiectasis: results and prognostic factors. Tuber Lung Dis 1996;77:168-72.

228 Balkanli K, Genç O, Dakak M, et al. Surgical management of bronchiectasis: analysis and short-term results in 238 patients. Eur J Cardiothorac Surg 2003;24:699-702.

229 Gorur R, Turut $H$, Yiyit N, et al. The influence of specific factors on postoperative morbidity in young adults with bronchiectasis. Heart Lung Circ 2011;20:468-72.
230 Zhang P, Jiang G, Ding J, et al. Surgical treatment of bronchiectasis: a retrospective analysis of 790 patients. Ann Thorac Surg 2010;90:246-50.

231 Zhou ZL, Zhao H, Li Y, et al. Completely thoracoscopic lobectomy for the surgical management of bronchiectasis. Chin Med J 2013;126:875-8.

232 Hiramatsu M, Shiraishi Y, Nakajima Y, et al. Risk factors that affect the surgical outcome in the management of focal bronchiectasis in a developed country. Ann Thorac Surg 2012;93:245-50.

233 Zhang $P$, Zhang $F$, Jiang $S$, et al. Video-assisted thoracic surgery for bronchiectasis. Ann Thorac Surg 2011;91:239-43.

234 Gursoy S, Ozturk AA, Ucvet A, et al. Surgical management of bronchiectasis: the indications and outcomes. Surg Today 2010;40:26-30.

235 Giovannetti R, Alifano M, Stefani A, et al. Surgical treatment of bronchiectasis: early and long-term results. Interact Cardiovasc Thorac Surg 2008;7:609-12.

236 Eren S, Esme H, Avci A. Risk factors affecting outcome and morbidity in the surgical management of bronchiectasis. J Thorac Cardiovasc Surg 2007;134:392-8.

237 Guerra MS, Miranda JA, Leal F, et al. Surgical treatment of bronchiectasis. [Portuguese, English];Tratamento cirurgico das bronquiectasias. Revista portuguesa de pneumologia 2007;13:691-701.

238 Warburton CJ, Corless JA. Surgery versus non-surgical treatment for bronchiectasis. Cochrane Database Syst Rev 2000:83.

239 Schneiter D, Meyer N, Lardinois D, et al. Surgery for non-localized bronchiectasis. $\mathrm{Br}$ Surg 2005;92:836-9.

240 Bagheri R, Haghi SZ, Fattahi Masoum SH, et al. Surgical management of bronchiectasis: analysis of 277 patients. Thorac Cardiovasc Surg 2010;58:291-4.

241 Orens JB, Estenne M, Arcasoy S, et al. International guidelines for the selection of lung transplant candidates: 2006 update--a consensus report from the Pulmonary Scientific Council of the International Society for Heart and Lung Transplantation. $J$ Heart Lung Transplant 2006;25:745-55.

242 Beirne PA, Banner NR, Khaghani A, et al. Lung transplantation for non-cystic fibrosis bronchiectasis: analysis of a 13-year experience. J Heart Lung Transplant 2005:24:1530-5

243 Nathan JA, Sharples LD, Exley AR, et al. The outcomes of lung transplantation in patients with bronchiectasis and antibody deficiency. J Heart Lung Transplant 2005:24:1517-21.

244 de Pablo A, López S, Ussetti P, et al. [Lung transplant therapy for suppurative diseases]. Arch Bronconeumol 2005:41:255-9.

245 Titman A, Rogers CA, Bonser RS, et al. Disease-specific survival benefit of lung transplantation in adults: a national cohort study. Am J Transplant 2009;9:1640-9.

246 Chang CC, Morris PS, Chang AB. Influenza vaccine for children and adults with bronchiectasis. Cochrane Database Syst Rev 2007:3:CD006218.

247 Poole P, Chacko EE, Wood-Baker R, et al. Influenza vaccine for patients with chronic obstructive pulmonary disease. Cochrane Database Syst Rev 2006:136:CD002733.

248 Chang CC, Singleton RJ, Morris PS, et al. Pneumococcal vaccines for children and adults with bronchiectasis. Cochrane Database Syst Rev 2009;2:CD006316.

249 Furumoto $A$, Ohkusa $Y$, Chen $M$, et al. Additive effect of pneumococcal vaccine and influenza vaccine on acute exacerbation in patients with chronic lung disease. Vaccine 2008;26:4284-9.

250 Moberley S, Holden J, Tatham DP, et al. Vaccines for preventing pneumococcal infection in adults. Cochrane Database Syst Rev 2013;61:CD000422.

251 Andrews NJ, Waight PA, George RC, et al. Impact and effectiveness of 23-valent pneumococcal polysaccharide vaccine against invasive pneumococcal disease in the elderly in England and Wales. Vaccine 2012;30:6802-8.

252 JCVI. JCVI statement on adult pneumococcal vaccination in the UK. London, 2015

253 Dupont M, Gacouin A, Lena $\mathrm{H}$, et al. Survival of patients with bronchiectasis after the first ICU stay for respiratory failure. Chest 2004;125:1815-20.

254 Phua J, Ang YL, See KC, et al. Noninvasive and invasive ventilation in acute respiratory failure associated with bronchiectasis. Intensive Care Med 2010;36:638-47.

255 Gursel G. Does coexistence with bronchiectasis influence intensive care unit outcome in patients with chronic obstructive pulmonary disease? Heart Lung 2006;35:58-65

256 Simonds AK, Elliott MW. Outcome of domiciliary nasal intermittent positive pressure ventilation in restrictive and obstructive disorders. Thorax 1995;50:604-9.

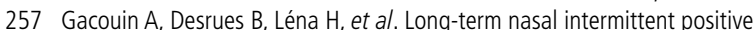
pressure ventilation (NIPPV) in sixteen consecutive patients with bronchiectasis: a retrospective study. Eur Respir J 1996;9:1246-50.

258 Benhamou D, Muir JF, Raspaud C, et al. Long-term efficiency of home nasal mask ventilation in patients with diffuse bronchiectasis and severe chronic respiratory failure: a case-control study. Chest 1997;112:1259-66.

259 Leger $\mathrm{P}$, Bedicam JM, Cornette $A$, et al. Nasal intermittent positive pressure ventilation. Long-term follow-up in patients with severe chronic respiratory insufficiency. Chest 1994;105:100-5.

260 Maa SH, Tsou TS, Wang KY, et al. Self-administered acupressure reduces the symptoms that limit daily activities in bronchiectasis patients: pilot study findings. $J$ Clin Nurs 2007:16:794-804.

261 Irons JY, Kenny DT, Chang AB. Singing for children and adults with bronchiectasis. Cochrane Database Syst Rev 2010;64:CD007729. 
262 Bartley J, Garrett J, Grant CC, et al. Could vitamin d have a potential antiinflammatory and anti-infective role in bronchiectasis? Curr Infect Dis Rep 2013;15:148-57.

263 Chalmers JD, McHugh BJ, Docherty C, et al. Vitamin-D deficiency is associated with chronic bacterial colonisation and disease severity in bronchiectasis. Thorax 2013;68:39-47.

264 Javadmoosavi SA, Shahabi Shahmiri S, Mostafapour E, et al. Comparison of the serum concentration of zinc in patients with bronchiectasis and control group. Iran Red Crescent Med J 2013;15:587-9.

265 Wilson CB, Jones PW, O'Leary CJ, et al. Validation of the St. George's Respiratory Questionnaire in bronchiectasis. Am J Respir Crit Care Med 1997;156:536-41.

266 McDonnell MJ, Jary HR, Perry A, et al. Non cystic fibrosis bronchiectasis: A longitudinal retrospective observational cohort study of Pseudomonas persistence and resistance. Respir Med 2015;109:716-26.

267 Fowler SJ, French J, Screaton NJ, et al. Nontuberculous mycobacteria in bronchiectasis: Prevalence and patient characteristics. Eur Respir J 2006;28:1204-10.

268 Kunst $\mathrm{H}$, Wickremasinghe $\mathrm{M}$, Wells $\mathrm{A}$, et al. Nontuberculous mycobacterial disease and Aspergillus-related lung disease in bronchiectasis. Eur Respir J 2006;28:352-7.

269 Goeminne PC, Nawrot TS, Ruttens D, et al. Mortality in non-cystic fibrosis bronchiectasis: a prospective cohort analysis. Respir Med 2014;108:287-96.

270 Loebinger MR, Wells AU, Hansell DM, et al. Mortality in bronchiectasis: a long-term study assessing the factors influencing survival. Eur Respir J 2009;34:843-9.

271 Evans SA, Turner SM, Bosch BJ, et al. Lung function in bronchiectasis: the influence of Pseudomonas aeruginosa. Eur Respir J 1996;9:1601-4.

272 Wilson CB, Jones PW, O'Leary CJ, et al. Effect of sputum bacteriology on the quality of life of patients with bronchiectasis. Eur Respir J 1997;10:1754-60.

273 Martínez-García MA, Perpiñá-Tordera M, Román-Sánchez P, et al. Quality-of-life determinants in patients with clinically stable bronchiectasis. Chest 2005;128:739-45.

274 Davies G, Wells AU, Doffman S, et al. The effect of Pseudomonas aeruginosa on pulmonary function in patients with bronchiectasis. Eur Respir J 2006:28:974-9.

275 Martínez-García MA, Soler-Cataluña JJ, Perpiñá-Tordera M, et al. Factors associated with lung function decline in adult patients with stable non-cystic fibrosis bronchiectasis. Chest 2007;132:1565-72.

276 Mirsaeidi M, Hadid W, Ericsoussi B, et al. Non-tuberculous mycobacterial disease is common in patients with non-cystic fibrosis bronchiectasis. Int J Infect Dis 2013;17:e1000-4.

277 Angrill J, Agustí C, De Celis R, et al. Bronchial inflammation and colonization in patients with clinically stable bronchiectasis. Am J Respir Crit Care Med 2001;164:1628-32.

278 Hill AT, Campbell EJ, Hill SL, et al. Association between airway bacterial load and markers of airway inflammation in patients with stable chronic bronchitis. Am J Med 2000;109:288-95.

279 Rogers GB, van der Gast CJ, Cuthbertson L, et al. Clinical measures of disease in adult non-CF bronchiectasis correlate with airway microbiota composition. Thorax 2013:68:731-7.

280 Tunney MM, Einarsson GG, Wei L, et al. Lung microbiota and bacterial abundance in patients with bronchiectasis when clinically stable and during exacerbation. Am J Respir Crit Care Med 2013;187:1118-26.

$281 \mathrm{Gao}$ YH, Guan WJ, Xu G, et al. The role of viral infection in pulmonary exacerbations of bronchiectasis in adults: a prospective study. Chest 2015;147:1635-43.

282 Máiz L, Vendrell M, Olveira C, et al. Prevalence and factors associated with isolation of Aspergillus and Candida from sputum in patients with non-cystic fibrosis bronchiectasis. Respiration 2015:89:396-403.

283 Orriols R, Hernando R, Ferrer A, et al. Eradication Therapy against Pseudomonas aeruginosa in Non-Cystic Fibrosis Bronchiectasis. Respiration 2015;90:299-305.

284 White L, Mirrani G, Grover M, et al. Outcomes of Pseudomonas eradication therapy in patients with non-cystic fibrosis bronchiectasis. Respir Med 2012;106:356-60.

285 Bilton D, Henig N, Morrissey B, et al. Addition of inhaled tobramycin to ciprofloxacin for acute exacerbations of Pseudomonas aeruginosa infection in adult bronchiectasis. Chest 2006;130:1503-10.

286 Murray MP, Turnbull K, Macquarrie S, et al. Assessing response to treatment of exacerbations of bronchiectasis in adults. Eur Respir J 2009:33:312-8.

287 Bedi P, Sidhu MK, Donaldson LS, et al. A prospective cohort study of the use of domiciliary intravenous antibiotics in bronchiectasis. NPJ Prim Care Respir Med 2014;24:14090.

288 Greenberger PA, Miller TP, Roberts M, et al. Allergic bronchopulmonary aspergillosis in patients with and without evidence of bronchiectasis. Ann Allergy 1993;70:333-8.

289 Greenberger PA, Bush RK, Demain JG, et al. Allergic Bronchopulmonary Aspergillosis. J Allergy Clin Immunol 2014;2:703-8.

290 Greenberger PA. Allergic bronchopulmonary aspergillosis. J Allergy Clin Immunol 2002;110:685-92.

291 Vlahakis NE, Aksamit TR. Diagnosis and treatment of allergic bronchopulmonary aspergillosis. Mayo Clin Proc 2001;76:930-8.

292 Agarwal R, Khan A, Aggarwal AN, et al. Role of inhaled corticosteroids in the management of serological allergic bronchopulmonary aspergillosis (ABPA). Intern Med 2011;50:855-60.
293 Imbeault B, Cormier Y. Usefulness of inhaled high-dose corticosteroids in allergic bronchopulmonary aspergillosis. Chest 1993;103:1614-7.

294 Cohen-Cymberknoh M, Blau H, Shoseyov D, et al. Intravenous monthly pulse methylprednisolone treatment for ABPA in patients with cystic fibrosis. J Cyst Fibros 2009;8:253-7

295 Wark PA, Hensley MJ, Saltos N, et al. Anti-inflammatory effect of itraconazole in stable allergic bronchopulmonary aspergillosis: a randomized controlled trial. $J$ Allergy Clin Immunol 2003;111:952-7.

296 Salez F, Brichet A, Desurmont S, et al. Effects of itraconazole therapy in allergic bronchopulmonary aspergillosis. Chest 1999:116:1665-8.

297 Wark PA, Gibson PG, Wilson AJ. Azoles for allergic bronchopulmonary aspergillosis associated with asthma. Cochrane Database Syst Rev 2004:42:CD001108.

298 Moreira AS, Silva D, Ferreira AR, et al. Antifungal treatment in allergic bronchopulmonary aspergillosis with and without cystic fibrosis: a systematic review. Clin Exp Allergy 2014;44:1210-27.

299 Chishimba L, Niven RM, Cooley J, et al. Voriconazole and posaconazole improve asthma severity in allergic bronchopulmonary aspergillosis and severe asthma with fungal sensitization. J Asthma 2012;49:423-33.

300 Quinti I, Soresina A, Guerra A, et al. Effectiveness of immunoglobulin replacement therapy on clinical outcome in patients with primary antibody deficiencies: results from a multicenter prospective cohort study. J Clin Immunol 2011:31:315-22

301 Quinti I, Soresina A, Spadaro G, et al. Long-term follow-up and outcome of a large cohort of patients with common variable immunodeficiency. J Clin Immunol 2007:27:308-16.

302 Busse PJ, Razvi S, Cunningham-Rundles C. Efficacy of intravenous immunoglobulin in the prevention of pneumonia in patients with common variable immunodeficiency. $J$ Allergy Clin Immunol 2002;109:1001-4.

303 de Gracia J, Vendrell M, Alvarez A, et al. Immunoglobulin therapy to control lung damage in patients with common variable immunodeficiency. Int Immunopharmacol 2004;4:745-53.

304 Roifman CM, Levison H, Gelfand EW. High-dose versus low-dose intravenous immunoglobulin in hypogammaglobulinaemia and chronic lung disease. Lancet 1987:1:1075-7.

305 Eijkhout HW, van Der Meer JW, Kallenberg CG, et al. The effect of two different dosages of intravenous immunoglobulin on the incidence of recurrent infections in patients with primary hypogammaglobulinemia. A randomized, double-blind, multicenter crossover trial. Ann Intern Med 2001;135:165-74.

306 Gathmann B, Mahlaoui N, Gérard L, et al. Clinical picture and treatment of 2212 patients with common variable immunodeficiency. J Allergy Clin Immunol 2014;134:116-26.

307 Orange JS, Grossman WJ, Navickis RJ, et al. Impact of trough IgG on pneumonia incidence in primary immunodeficiency: A meta-analysis of clinical studies. Clin Immunol 2010;137:21-30.

308 Lucas M, Lee M, Lortan J, et al. Infection outcomes in patients with common variable immunodeficiency disorders: relationship to immunoglobulin therapy over 22 years. J Allergy Clin Immunol 2010;125:1354-60.

309 Perez EE, Orange JS, Bonilla F, et al. Update on the use of immunoglobulin in human disease: a review of evidence. J Allergy Clin Immunol 2017;139:S1-S46.

310 Shehata N, Palda V, Bowen T, et al. The use of immunoglobulin therapy for patients with primary immune deficiency: an evidence-based practice guideline. Transfus Med Rev 2010;24:S28-50.

311 Rich $A L$, Le Jeune IR, McDermott $L$, et al. Serial lung function tests in primary immune deficiency. Clin Exp Immuno/ 2008;151:110-3.

312 Chen Y, Stirling RG, Paul E, et al. Longitudinal decline in lung function in patients with primary immunoglobulin deficiencies. J Allergy Clin Immunol 2011;127:1414-7.

313 Gregersen S, Aaløkken TM, Mynarek G, et al. High resolution computed tomography and pulmonary function in common variable immunodeficiency. Respir Med 2009;103:873-80.

314 Lee AL, Button BM, Denehy L, et al. BM. Gastro-oesophageal reflux in noncystic fibrosis bronchiectasis. Pulm Med 2011;2011:1-6.

315 Tsang KW, Lam WK, Kwok E, et al. Helicobacter pylori and upper gastrointestina symptoms in bronchiectasis. Eur Respir J 1999;14:1345-50.

316 Tsang KW, Lam SK, Lam WK, et al. High seroprevalence of Helicobacter pylori in active bronchiectasis. Am J Respir Crit Care Med 1998;158:1047-51.

317 Najafizadeh K, Saloor M, Fallah Tafti S, et al. Helicobacter pylori seroprevalence in patients with bronchiectasis. Tanaffos 2006:5:25-9.

318 Angrill J, Sánchez N, Agustí C, et al. Does Helicobacter pylori have a pathogenic role in bronchiectasis? Respir Med 2006;100:1202-7.

319 NICE. Gastro-oesophageal reflux disease and dyspepsia in adults: investigation and management. NICE clinical guideline. London, 2014

320 Guilemany JM, Alobid I, Angrill J, et al. The impact of bronchiectasis associated to sinonasal disease on quality of life. Respir Med 2006;100:1997-2003.

321 Guilemany JM, Mariño-Sánchez FS, Angrill J, et al. The importance of smell in patients with bronchiectasis. Respir Med 2011;105:44-9. 
322 Guilemany JM, Angrill J, Alobid I, et al. United airways: the impact of chronic rhinosinusitis and nasal polyps in bronchiectasic patient's quality of life. Allergy 2009;64:1524-9.

323 Guilemany JM, Angrill J, Alobid I, et al. United airways again: high prevalence of rhinosinusitis and nasal polyps in bronchiectasis. Allergy 2009;64:790-7.

324 Fokkens WJ, Lund VJ, Mullol J, et al. European position paper on Rhinosinusitis and Nasal Polyps 2012. Rhino/ Supp/ 2012;23:3.

325 Scadding GK, Durham SR, Mirakian R, et al. BSACI guidelines for the management of rhinosinusitis and nasal polyposis. Clin Exp Allergy 2008;38:260-75.

326 Tomooka LT, Murphy C, Davidson TM. Clinical study and literature review of nasal irrigation. Laryngoscope 2000;110:1189-93.

327 Fowler PD, Gazis AG, Page SR, et al. A randomized double-blind study to compare the effects of nasal fluticasone and betamethasone on the hypothalamo-pituitaryadrenal axis and bone turnover in patients with nasal polyposis. Clin Otolaryngol Allied Sci 2002;27:489-93.

328 Cervin A, Wallwork B. Efficacy and safety of long-term antibiotics (macrolides) for the treatment of chronic rhinosinusitis. Curr Allergy Asthma Rep 2014;14:416.

329 Souders CR. Bronchiectasis and its management; a report of 277 cases. Dis Chest 1949;16:381-407.

330 Hurst JR, Elborn JS, De Soyza A, et al. COPD-bronchiectasis overlap syndrome. Eur Respir J 2015:45:310-3.

331 O'Brien C, Guest PJ, Hill SL, et al. Physiological and radiological characterisation of patients diagnosed with chronic obstructive pulmonary disease in primary care. Thorax 2000;55:635-42.

332 Patel IS, Vlahos I, Wilkinson TM, et al. Bronchiectasis, exacerbation indices, and inflammation in chronic obstructive pulmonary disease. Am J Respir Crit Care Med 2004;170:400-7.

333 McGuinness G, Naidich DP, Garay S, et al. AIDS associated bronchiectasis: CT features. J Comput Assist Tomogr 1993;17:260-6.

334 King MA, Neal DE, St John R, et al. Bronchial dilatation in patients with HIV infection: CT assessment and correlation with pulmonary function tests and findings at bronchoalveolar lavage. AJR Am J Roentgenol 1997;168:1535-40.

335 Camus PCT. Bronchiectasis associated with inflammatory bowel disease. Eur Respir 2011;52:163-77.

336 Goeminne PC, Verschueren P, Scheers H, et al. Safety of immunomodulatory therapy in patients with bronchiectasis associated with rheumatic disease and IBD: a retrospective and cohort analysis. Clin Rheumatol 2012;31:367-73.

337 Geri G, Dadoun S, Bui T, et al. Risk of infections in bronchiectasis during diseasemodifying treatment and biologics for rheumatic diseases. BMC Infect Dis 2011;11:304.

338 McDonnell MJ, Aliberti S, Goeminne PC, et al. Comorbidities and the risk of mortality in patients with bronchiectasis: an international multicentre cohort study. Lancet Respir Med 2016:4:969-79.

339 Spinou A, Fragkos KC, Lee KK, et al. The validity of health-related quality of life questionnaires in bronchiectasis: a systematic review and meta-analysis. Thorax 2016:71:683-94.

340 Quittner AL, O'Donnell AE, Salathe MA, et al. Quality of Life QuestionnaireBronchiectasis: final psychometric analyses and determination of minimal important difference scores. Thorax 2015;70:12-20.

341 Hill AT. BTS Quality Standards for clinically significant bronchiectasis in adults. 2012.

342 Barker AF, Couch L, Fiel SB, et al. Tobramycin solution for inhalation reduces sputum Pseudomonas aeruginosa density in bronchiectasis. Am J Respir Crit Care Med 2000;162:481-5.

343 Drobnic ME, Suñé $P$, Montoro JB, et al. Inhaled tobramycin in non-cystic fibrosis patients with bronchiectasis and chronic bronchial infection with Pseudomonas aeruginosa. Ann Pharmacother 2005;39:39-44.

344 Rayner CF, Tillotson G, Cole PJ, et al. Efficacy and safety of long-term ciprofloxacin in the management of severe bronchiectasis. J Antimicrob Chemother 1994;34:149-56

345 Gillham MI, Sundaram S, Laughton CR, et al. Variable antibiotic susceptibility in populations of Pseudomonas aeruginosa infecting patients with bronchiectasis. J Antimicrob Chemother 2009:63:728-32.

346 Saiman L, Siegel JD, LiPuma JJ, et al. Infection prevention and control guideline for cystic fibrosis: 2013 update. Infect Control Hosp Epidemiol 2014;35:S1-67.

347 Bryant JM, Grogono DM, Greaves D, et al. Whole-genome sequencing to identify transmission of Mycobacterium abscessus between patients with cystic fibrosis: a retrospective cohort study. Lancet 2013;381:1551-60.

348 De Soyza A, Perry A, Hall AJ, et al. Molecular epidemiological analysis suggests cross infection with Pseudomonas aeruginosa is rare in non-cystic fibrosis bronchiectasis. Eur Respir J 2014:43:900-3

349 Pujana I, Gallego L, Martín G, et al. Epidemiological analysis of sequential Pseudomonas aeruginosa isolates from chronic bronchiectasis patients without cystic fibrosis. J Clin Microbiol 1999;37:2071-3.
350 Valderrey AD, Pozuelo MJ, Jiménez PA, et al. Chronic colonization by Pseudomonas aeruginosa of patients with obstructive lung diseases: cystic fibrosis, bronchiectasis, and chronic obstructive pulmonary disease. Diagn Microbiol Infect Dis 2010;68:20-7.

351 Hilliam Y, Moore MP, Lamont IL, et al. Pseudomonas aeruginosa adaptation and diversification in the non-cystic fibrosis bronchiectasis lung. Eur Respir $J$ 2017:49:1602108.

352 Mitchelmore PJ, Randall J, Bull MJ, et al. Molecular epidemiology of Pseudomonas aeruginosa in an unsegregated bronchiectasis cohort sharing hospital facilities with a cystic fibrosis cohort. Thorax 2017:thoraxjnl-2016-209889 [Epub ahead of print26 Aug 2017].

353 lakhiaeva E, McNulty S, Brown Elliott BA, et al. Mycobacterial interspersed repetitiveunit-variable-number tandem-repeat (MIRU-VNTR) genotyping of mycobacterium intracellulare for strain comparison with establishment of a PCR-based database. J Clin Microbio/ 2013;51:409-16.

354 Konietzko NF, Carton RW, Leroy EP. Causes of death in patients with bronchiectasis. Am Rev Respir Dis 1969;100:852-8.

355 Ellis DA, Thornley PE, Wightman AJ et al. Present outlook in bronchiectasis: clinical and social study and review of factors influencing prognosis. Thorax 1981;36(9): 659-4.

356 Aliberti S, Lonni S, Dore S, et al. Clinical phenotypes in adult patients with bronchiectasis. Eur Respir J 2016;47:1113-22.

357 Agasthian T. Results of surgery for bronchiectasis and pulmonary abscesses. Thorac Surg Clin 2012;22:333-44.

358 Lee JK, Lee J, Park SS, et al. Effect of inhalers on the development of haemoptysis in patients with non-cystic fibrosis bronchiectasis. Int I Tuberc Lung Dis 2014;18:363-70.

359 King PT, Holdsworth SR, Farmer M, et al. Chest pain and exacerbations of bronchiectasis. Int J Gen Med 2012;5:1019-24.

360 Hill AT, Routh C, Welham S. National BTS bronchiectasis audit 2012: is the quality standard being adhered to in adult secondary care? Thorax 2014;69:292-4.

361 Tonelli P.A morphologic study of nodular lung carcinomas and their possible pathogenesis from a cluster of non-obstructive bronchiectasis. Lung Cancer 1997;17:135-45.

362 Chung WS, Lin CL, Hsu WH, et al. Increased risk of lung cancer among patients with bronchiectasis: a nationwide cohort study. QJM 2016;109:17-25.

363 Kim YW, Jin KN, Heo EY, et al. The association between combined non-cystic fibrosis bronchiectasis and lung cancer in patients with chronic obstructive lung disease. Int J Chron Obstruct Pulmon Dis 2015:10:873-9.

364 Navaratnam V, Millett ER, Hurst JR, et al. Bronchiectasis and the risk of cardiovascular disease: a population-based study. Thorax 2017;72:161-6.

365 Evans IE, Bedi P, Quinn TM, et al. Bronchiectasis severity is an independent risk factor for vascular disease in a bronchiectasis cohort. Chest 2017:151:383-8.

366 Saleh AD, Kwok B, Brown JS, et al. Correlates and assessment of excess cardiovascular risk in bronchiectasis. Eur Respir J 2017;50:1701127.

367 Devaraj A, Wells AU, Meister MG, et al. Pulmonary hypertension in patients with bronchiectasis: prognostic significance of CT signs. AJR Am J Roentgenol 2011;196:1300-4.

368 Alzeer AH, Al-Mobeirek AF, Al-Otair HA, et al. Right and left ventricular function and pulmonary artery pressure in patients with bronchiectasis. Chest 2008:133:468-73.

369 O'Leary CJ, Wilson CB, Hansell DM, et al. Relationship between psychological well-being and lung health status in patients with bronchiectasis. Respir Med 2002;96:686-92.

370 Olveira C, Olveira G, Gaspar I, et al. Depression and anxiety symptoms in bronchiectasis: associations with health-related quality of life. Qual Life Res 2013:22:597-605.

371 Ryu YJ, Chun EM, Lee JH, et al. Prevalence of depression and anxiety in outpatients with chronic airway lung disease. Korean J Intern Med 2010;25:51-7.

372 Girón Moreno RM, Fernandes Vasconcelos G, Cisneros C, et al. Presence of anxiety and depression in patients with bronchiectasis unrelated to cystic fibrosis. Arch Bronconeumol 2013:49:415-20.

373 Rees J, Tedd H, De Soyza A. Managing urinary incontinence in adults with bronchiectasis. Br J Nurs 2013:22:S15S18-6.

374 Gale NS, Bolton CE, Duckers JM, et al. Systemic comorbidities in bronchiectasis. Chron Respir Dis 2012:9:231-8.

375 Rajagopala S, Ramakrishnan A, Bantwal G, et al. Adrenal insufficiency in patients with stable non-cystic fibrosis bronchiectasis. Indian J Med Res 2014;139:393-401.

376 Onen ZP, Gulbay BE, Sen E, et al. Analysis of the factors related to mortality in patients with bronchiectasis. Respir Med 2007;101:1390-7.

377 Olveira G, Olveira C, Gaspar I, et al. Fat-free mass depletion and inflammation in patients with bronchiectasis. J Acad Nutr Diet 2012;112:1999-2006.

378 Hester KL, Macfarlane JG, Tedd H, et al. Fatigue in bronchiectasis. QJM 2012;105:235-40.

379 King PT, Holdsworth SR, Freezer NJ, et al. Characterisation of the onset and presenting clinical features of adult bronchiectasis. Respir Med 2006;100:2183-9.

380 Gregersen S, Aaløkken TM, Mynarek G, et al. Development of pulmonary abnormalities in patients with common variable immunodeficiency: associations with clinical and immunologic factors. Ann Allergy Asthma Immunol 2010:104:503-10. 